The Astronomical Journal, 129:1534-1563, 2005 March

(C) 2005. The American Astronomical Society. All rights reserved. Printed in U.S.A.

\title{
THE ORION NEBULA IN THE MID-INFRARED
}

\author{
M. Robberto, ${ }^{1}$ S. V. W. Beckwith, and N. Panagia ${ }^{1}$ \\ Space Telescope Science Institute, 4300 San Martin Drive, Baltimore, MD 21218 \\ S. G. PATEL ${ }^{2}$ \\ University of California, Santa Cruz, 1156 High Street, Santa Cruz, CA 95064 \\ T. M. Herbst and S. Ligori \\ Max-Planck-Institut für Astronomie, Königstuhl 17, D-69117 Heidelberg, Germany \\ AND \\ A. Custo, ${ }^{3}$ P. Boccacci, and M. Bertero \\ INFM and Dipartimento di Informatica e Scienze dell'Informazione, Università di Genova, \\ Via Dodecaneso 35, I-16146 Genoa, Italy \\ Received 2004 March 31; accepted 2004 December 13
}

\begin{abstract}
We present two wide-field $\left(\approx 5^{\prime} \times 3.5\right)$, diffraction-limited $(\lambda / D \simeq 0$ '.5 at $10 \mu \mathrm{m})$, broadband 10 and $20 \mu \mathrm{m}$ images of the Orion Nebula, plus six $7-13 \mu \mathrm{m}$ narrowband $(\lambda / \Delta \lambda \simeq 1)$ images of the BN/KL complex taken at the $3.8 \mathrm{~m}$ UKIRT telescope with the MPIA MAX camera. The wide-field images, centered on the Trapezium and BN/KL regions, are mosaics of $35^{\prime \prime} \times 35^{\prime \prime}$ frames obtained with standard chopping and nodding techniques and reconstructed using a new restoration method developed for this project. They show the filamentary structure of the dust emission from the walls of the $\mathrm{H}$ II region and reveal a new remarkable group of arclike structures $\approx 1^{\prime}$ to the south of the Trapezium. The morphology of the Ney-Allen Nebula, produced by wind-wind interaction in the vicinity of the Trapezium stars, suggests a complex kinematical structure at the center of the cluster. We find indications that one of the most massive members of the cluster, the B0.5 V star $\theta^{1}$ Ori D, is surrounded by a photoevaporated circumstellar disk. Among the four historic Trapezium OB stars, this is the only one without a binary companion, suggesting that stellar multiplicity and the presence of massive circumstellar disks may be mutually exclusive. In what concerns the BN/KL complex, we find evidence for extended optically thin silicate emission on top of the deep $10 \mu \mathrm{m}$ absorption feature. Assuming a simple two-component model, we map with $\simeq 0$.'5 spatial resolution the foreground optical depth, color temperature, and mid-IR luminosity of the embedded sources. We resolve a conspicuous point source at the location of the IRc2-A knot, approximately 0 ".5 north of the deeply embedded H II region "I." We analyze the spectral profile of the $10 \mu \mathrm{m}$ silicate absorption feature and find indication for grain crystallization in the harsh nebular environment. In the OMC-1 South region, we detect several point sources and discuss their association with the mass-loss phenomenology observed at optical and millimeter wavelengths. Finally, we list the position and photometry of 177 point sources, the large majority of which are detected for the first time in the mid-IR. Twenty-two of them lack a counterpart at shorter wavelengths and are therefore candidates for deeply embedded protostars. The comparison of photometric data obtained at two different epochs reveals that source variability at $10 \mu \mathrm{m}$ is present up to a level of $\approx 1$ mag on a timescale of $\sim 2 \mathrm{yr}$. With the possible exception of a pair of OB stars, all point sources detected at shorter wavelengths display $10 \mu \mathrm{m}$ emission well above the photospheric level, which we attribute to disk circumstellar emission. The recent model of Robberto et al. provides the simplest explanation for the observed mid-IR excess.
\end{abstract}

Key words: infrared: general — ISM: individual (Orion Nebula) - methods: data analysis planetary systems: protoplanetary disks — stars: formation

\section{INTRODUCTION}

The Great Orion Nebula (M42=NGC 1976), with its associated cluster of pre-main-sequence stars, represents the nearest $(d \simeq 450 \mathrm{pc})$ and best-studied episode of massive star formation (Lada \& Lada 2003). The stellar cluster, distributed on a volume of $\sim 3$ pc radius (Hillenbrand \& Hartmann 1998), contains approximately 2500 stars $\simeq 10^{6} \mathrm{yr}$ old spanning the mass range 45-0.02 $M_{\odot}$ (Hillenbrand 1997; Hillenbrand \& Carpenter 2000).

\footnotetext{
${ }^{1}$ Affiliated with the Space Telescope Division of the European Space Agency, ESTEC, Noordwijk, Netherlands.

${ }^{2}$ Space Telescope Science Institute Summer Student Program participant.

${ }^{3}$ Current address: Massachusetts Institute of Technology, Computer Science and Artificial Intelligence Laboratory, 32 Vassar Street, Cambridge, MA 02139.
}

There is clear evidence of mass segregation, with the most massive OB stars (the Trapezium system) concentrated in the central $\approx 0.15 \mathrm{pc}$, where the stellar density approaches $2 \times 10^{4}$ stars $\mathrm{pc}^{-3}$. Ultraviolet radiation from the Trapezium stars, mostly the O6.5 V star $\theta^{1}$ Ori $\mathrm{C}$, has produced the Great Orion Nebula (O’Dell 2001a, 2001b), a blister cavity excavated on the surface of the parental molecular cloud OMC-1. The same UV radiation also photoablates the accreting circumstellar disks of the nearest stars, producing the characteristic photoionized "proplyds" (Laques \& Vidal 1979; O’Dell et al. 1993). Proplyds provide the most compelling evidence that the star formation process can be dramatically affected by the mutual interaction between cluster members.

The Orion Nebula Cluster, especially the core region (the Trapezium cluster), has been investigated by several authors using 
TABLE 1

Main Observational Parameters

\begin{tabular}{|c|c|c|c|c|c|}
\hline Night & Filter & $\begin{array}{l}\text { Integration Time } \\
(\mathrm{ms})\end{array}$ & $\begin{array}{c}\text { Chop Frequency } \\
(\mathrm{Hz})\end{array}$ & $\begin{array}{l}\text { Integration Time per Frame } \\
\text { (s) }\end{array}$ & $\begin{array}{c}\text { Chop Throw } \\
\text { (arcsec) }\end{array}$ \\
\hline 1998 Nov $27 \ldots \ldots \ldots \ldots$ & $N$ & 20.2 & 4.2 & 20.07 & $9.80(\mathrm{~N}-\mathrm{S})$ \\
\hline 1998 Nov $27 \ldots \ldots \ldots \ldots$ & $N$ & 20.2 & 4.2 & 40.14 & $18.55(\mathrm{~N}-\mathrm{S})$ \\
\hline 2000 Dec 18 & $N$ & 9.9 & 3.9 & 25.36 & $24.64(\mathrm{E}-\mathrm{W})$ \\
\hline 2000 Dec 19 & $N$ & 9.9 & 3.9 & 25.36 & $24.64(\mathrm{E}-\mathrm{W})$ \\
\hline 2000 Dec 19 & $Q$ & 9.9 & 3.9 & 12.68 & $24.64(\mathrm{E}-\mathrm{W})$ \\
\hline 2000 Dec 18 & Silicates & 20.2 & 4.12 & 12.944 & $24.64(\mathrm{E}-\mathrm{W})$ \\
\hline
\end{tabular}

data collected at visible (Prosser et al. 1994; Hillenbrand 1997; Robberto et al. 2004) and near-IR wavelengths (McCaughrean \& Stauffer 1994; Hillenbrand \& Carpenter 2000; Lucas \& Roche 2000; Luhman et al. 2000; Lada et al. 2000). Since circumstellar disks emit in the IR the energy they "passively" absorb from their central stars or "actively" produce by the viscous dissipation processes associated with mass accretion, there is a need for similar observations at longer wavelengths, in particular in the mid-IR $(\lambda \simeq 5-20 \mu \mathrm{m})$, where ground-based telescopes can reach subarcsecond spatial resolution. Mid-IR observations have also the potential of discovering objects more deeply embedded within the molecular cloud, otherwise invisible at shorter wavelengths (with the possible exception of hard X-rays), and eventually identifying sites where protostellar contraction is still ongoing. Moreover, mid-IR data may also provide complementary information on the structure of the $\mathrm{H}$ II region through the diffuse emission of the dust grains and polycyclic aromatic hydrocarbon (PAH) molecules mixed with the ionized gas or located in the surrounding photodissociation region (PDR).

The first $3.5 \mu \mathrm{m}$ image of the Trapezium cluster taken with a panoramic detector was presented, but only briefly discussed, by McCaughrean et al. (1996). A more complete study of the Trapezium cluster at this wavelength has been published by Lada et al. (2000). They found that $80 \%-85 \%$ of stars are surrounded by circumstellar disks, in agreement with earlier studies made at near-IR wavelengths, and identify 78 sources with extremely red colors that they classify as candidate protostars.

At wavelengths $\lambda>3.5 \mu \mathrm{m}$, the region remains largely unexplored. Maps made with single-element bolometers in the early days of IR astronomy revealed two prominent sources, the BN/ KL complex (Becklin \& Neugebauer 1967; Kleinman \& Low 1967) and the Ney-Allen Nebula in the vicinity of the Trapezium (Ney \& Allen 1969). Both fields have been widely investigated with increasing sensitivity and spatial and spectral resolution. Images at 10 and $20 \mu \mathrm{m}$ made with panoramic detectors have been published by Gezari et al. (1998), Greenhill et al. (2004), and Shuping et al. (2004) for BN/KL and by Hayward et al. (1994) for the Ney-Allen Nebula. Smith et al. (2004) have recently presented an image of the OMC-1 South region.

We have undertaken a large observational program on the Orion Nebula using the mid-IR camera MAX on the UKIRT ${ }^{4}$ telescope. Our research program consists of two parts: first, to observe at $10 \mu \mathrm{m}$ with relatively long integration times the most prominent proplyds in order to detect and resolve their structure in the mid-IR, and second, to map the entire nebular core, i.e., an area of approximately $5^{\prime} \times 5^{\prime}$ covering both the Ney-Allen and $\mathrm{BN} / \mathrm{KL}$ regions, with shorter integration times. In this paper we present the results relative to this second study, complemented

\footnotetext{
4 The United Kingdom Infrared Telescope is operated by the Joint Astronomy Centre on behalf of the UK Particle Physics and Astronomy Research Council.
}

by narrowband observations of the BN/KL complex. In $\S 2$ we present our observations and our data reduction strategy, based on a reconstruction method originally developed for this project. In $\S 3$, we present our main results, the 10 and $20 \mu$ m wide-field images and the photometric catalog of 177 point sources detected at $10 \mu \mathrm{m}$. In $\S 4$, we discuss the overall structure of the nebula ( $\S 4.1)$, the Ney-Allen region ( 44.2 ), the $\mathrm{BN} / \mathrm{KL}$ complex (§ 4.3), the OMC-1 South region ( $(4.4)$, and finally the main properties of our sample of point sources $(\S 4.5)$. In $\S 5$, we summarize our findings.

\section{OBSERVATIONS AND DATA REDUCTION}

\subsection{Data Acquisition}

The wide-field maps presented in this paper were obtained combining observations made in 1998 November and 2000 December at the $3.8 \mathrm{~m}$ United Kingdom Infrared Telescope (UKIRT) on Mauna Kea, Hawaii. We used MAX ${ }^{5}$ (Robberto \& Herbst 1998), a mid-IR imager based on a Rockwell $128 \times$ $128 \mathrm{Si}$ :As blocked impurity band detector optimized for high background observations. MAX's optics delivered a plate scale of 0 .'26, corresponding to an approximately $35^{\prime \prime} \times 35^{\prime \prime}$ field of view. Images have been obtained through broad $N$-band and $Q$-band filters. M. Cohen provided detailed modeling of our photometric system, including typical Mauna Kea transmission, optics throughput, and detector response. For an A0 V star, the $N$-band filter has an effective wavelength $\lambda_{10}=10.1 \mu \mathrm{m}$ and $\Delta \lambda=3.13 \mu \mathrm{m}$, whereas the $Q$-band filter has $\lambda_{20}=19.7 \mu \mathrm{m}$ and $\Delta \lambda=2.44 \mu \mathrm{m}$. The narrowband images were obtained on 2000 December 18, through the standard set of silicate filters centered at 7.7, 8.7, 9.8, 10.2, 11.2, and $12.4 \mu \mathrm{m}$ (see Robberto $\&$ Herbst 1998 for the detailed filter parameters).

UKIRT is equipped with tip-tilt adaptive correction at the secondary mirror (Hawarden et al. 1999). Exploiting this capability, MAX routinely reaches diffraction-limited performances at $\lambda \geq 8 \mu \mathrm{m}$ with $\lambda / D \simeq 0$ " 5 . The guide system, however, only allows for chopping throws of at most $30^{\prime \prime}$ (a maximum of $\simeq 20^{\prime \prime}$ was in fact suggested for the $\simeq 5 \mathrm{~Hz}$ chopping frequency we used). Given the extent of the Orion Nebula and the MAX sensitivity, we could not chop onto empty sky areas for background subtraction. We experimented with the use of sky frames taken several arcminutes away, nodding the telescope at a frequency $\simeq 0.1 \mathrm{~Hz}$, constrained by the telescope pointing and tip-tilt reference star acquisition, but this method produced significant losses of sensitivity and observing efficiency. We therefore opted for the conventional chopping and nodding technique, adopting an observing strategy optimized for image post-processing, namely, the reconstruction method envisioned in Bertero et al. (2000) and references therein. In Table 1, we list the main observing

\footnotetext{
5 The instrument has been decommissioned since 2000 December. The $20 \mu \mathrm{m}$ mosaic represents the very last set of images taken by MAX.
} 
TABLE 2

Calibrators for Narrowband Imaging

\begin{tabular}{|c|c|c|c|c|c|c|}
\hline Source & $7.7 \mu \mathrm{m}$ & $8.7 \mu \mathrm{m}$ & $9.8 \mu \mathrm{m}$ & $10.2 \mu \mathrm{m}$ & $11.2 \mu \mathrm{m}$ & $12.4 \mu \mathrm{m}$ \\
\hline HR $1040 \ldots \ldots \ldots \ldots . . . . . .$. & 2.55 & 2.55 & 2.55 & 2.55 & 2.55 & 2.55 \\
\hline HR $1457 \ldots \ldots \ldots \ldots . . . . . .$. & -2.97 & -2.94 & -3.00 & -3.04 & -3.07 & -3.07 \\
\hline HR $1713 \ldots \ldots \ldots \ldots . . . . .$. & 0.06 & 0.06 & 0.06 & 0.06 & 0.06 & 0.06 \\
\hline HR $4069 \ldots \ldots \ldots \ldots . . . . . .$. & -0.84 & -0.83 & -0.91 & -0.96 & -1.00 & -1.00 \\
\hline HR $2943 \ldots \ldots \ldots \ldots \ldots$ & -0.77 & -0.77 & -0.76 & -0.76 & -0.76 & -0.76 \\
\hline HR $5340 \ldots \ldots \ldots \ldots \ldots$ & -3.11 & -3.10 & -3.14 & -3.17 & -3.18 & -3.18 \\
\hline$F_{m=0}(\mathrm{Jy}) \ldots \ldots \ldots$ & 63.56 & 50.86 & 41.64 & 36.67 & 28.94 & 25.28 \\
\hline
\end{tabular}

parameters: night of the observation, filter, integration time per single frame, chopping frequency, total exposure time on the main beam per each nodding cycle, and chopping throw amplitude and direction.

We scanned the survey region both in declination and right ascension using different chopping and nodding amplitudes. At each step, the telescope was offset (nodding) by an amount equal to the chopping amplitude; i.e., the new pointing position of the telescope on the main beam was coincident with the position imaged in the previous offset beam. Directions and amplitudes of chopping and nodding were carefully matched and aligned with the orientation of the array columns or rows. Because of $\mathrm{a} \sim 1^{\circ}$ rotation of the detector with respect to the equatorial coordinates, the chopping and nodding directions were not exactly coincident with the north-south or east-west axes. Therefore, the combined rows or columns have a slight drift with respect to the scan direction. The setup process and observing strategy is detailed further in Bertero et al. (2000).

Scans were typically $\sim 4^{\prime}$ long, with a small side overlay ( $\sim 10$ pixels) to maximize field coverage. Since in the two runs the camera was mounted on different focal positions of the telescope, images are rotated by $90^{\circ}$ between the two runs. The rotation of the optical path allows us to easily individuate ghost images produced by the brightest sources, $\mathrm{BN}$ in particular.

Observations were generally performed up to an air mass $z=2$ under excellent weather conditions. Absolute flux calibration in the $N$ band has been obtained by observing at various air masses the following standard stars: HR $337([N]=-2.06)$, HR 1040 $([N]=2.55)$, HR $1713([N]=0.06), \operatorname{HR} 2061([N]=-5.13)$, HR $4069([N]=-0.94)$, HR $2943([N]=-0.76)$, HR 4554 $([N]=2.32)$, and HR $5340([N]=-3.17)$. Our absolute flux calibration assumes for $\alpha \operatorname{Lyr}[N]=0$ with a flux $F=1.116 \pm$ $0.015 \times 10^{-16} \mathrm{~W} \mathrm{~cm}^{-2} \mu \mathrm{m}^{-1}$ at $\lambda_{10}$, corresponding to $37.9 \mathrm{Jy}$. Calibration at $20 \mu \mathrm{m}$ was based on HR $1760([Q]=-0.30)$, HR $2061([Q]=5.7)$, and HR $4069([Q]=-1.00)$. The zeromagnitude flux in this case is $F=7.887 \pm 0.011 \times 10^{-18} \mathrm{~W} \mathrm{~cm}^{-2}$ $\mu \mathrm{m}^{-1}$ at $\lambda_{20}$, corresponding to $10.2 \mathrm{Jy}$. The narrowband images of BN were calibrated against HR 1040, 1457, 1713, 4069, 2943 and 5340, with magnitudes and zero points given in Table 2. It is clear that the depth of our images is not uniform, since the total integration time in each scan is a function of the adopted pattern (amplitude of the chopping throws and offset between adjacent rows/columns), air mass, repeated or discarded frames, etc. Moreover, in the final images, part of the field has been observed on more than one night. In general, we estimate that the average integration time in the $N$ band is on the order of $200 \mathrm{~s}$. Concerning the narrowband images, the total integration time is $103.5 \mathrm{~s}$ at all wavelengths except the $8.7 \mu \mathrm{m}$ band, where it is $102.5 \mathrm{~s}$.

As a final remark, we note that we were able to obtain several scans of the region only at $10 \mu \mathrm{m}$. The $20 \mu \mathrm{m}$ data were all collected in one night, with only one chopping and nodding amplitude. The final mosaic is therefore shallower and potentially more affected by reconstruction artifacts.

\subsection{Data Reduction}

The raw images were first median-filtered to remove a small number $(\approx 15)$ of dead pixels and occasional extra noise in the frames containing the saturated BN peak. Cleaned frames were then normalized to the same integration time and combined to form chopped and nodded pairs. The final combination in the wide-area mosaic required a special effort, described in the following section.

\subsubsection{Mosaics}

All chopped and nodded pairs taken on the same night were combined into rows or columns and then into a single mosaic. This operation was performed using MOSAIC, an IDL package designed for mid-IR data and publicly available on the Internet (Varosi \& Gezari 1993). The images were combined by integer pixels, rounding the coordinates of the FITS header (accurate to $\simeq 0^{\prime \prime} 1$ ) to the nearest value. Figure 1 illustrates the mosaicking process using images directly grabbed from the MOSAIC output windows. The mosaics contain for each source two negative counterparts at the position of the two offset beams. Hence, we needed to reconstruct a true image.

The restoration method we have used was specifically developed for this project. The theoretical basis has been illustrated in a number of papers (Bertero et al. 1998, 1999, 2000); thus, we provide here only a brief outline. Since the problem is underconstrained, it admits infinite solutions. In particular, the set of solutions whose chopped and nodded images are identically zero constitutes the null space, infinite dimensional, of the chopping and nodding operator. Any linear combination of these functions can be added to a reconstructed image without affecting the observed chopped and nodded image. In the null space it is possible to define a metric and apply minimization processes. Our strategy is to search for the minimal positive solution, assuming the celestial signal cannot be negative. The implementation of the algorithm is based on the Landweber method (Bertero \& Boccacci 1998). It is iterative and therefore produces an approximate solution at each step. The number of iterations needs to be optimized to control noise propagation, and our criterion has been described in Bertero et al. (2000). When applied to real images, it produces excellent results. In some situations, however, it may generate artifacts, e.g., artificial ghost images of the stars (appearing as dark images over a bright background or as bright images over a dark background) or discontinuities in the background level. The location and periodicity of the artifacts are related to the values of image size and chopping amplitude through the mathematical structure of the problem (Bertero et al. 1999). For our $10 \mu \mathrm{m}$ 

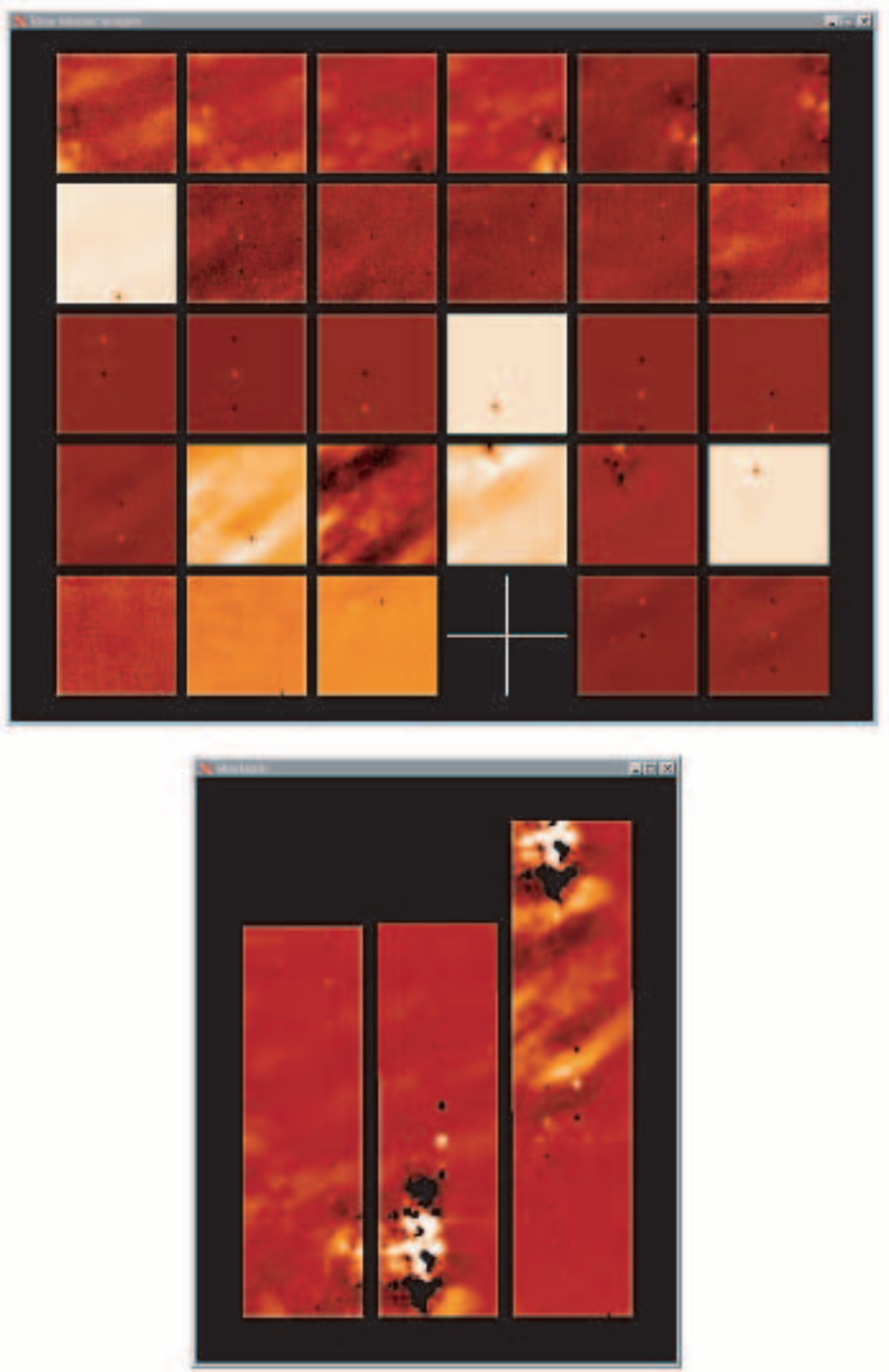
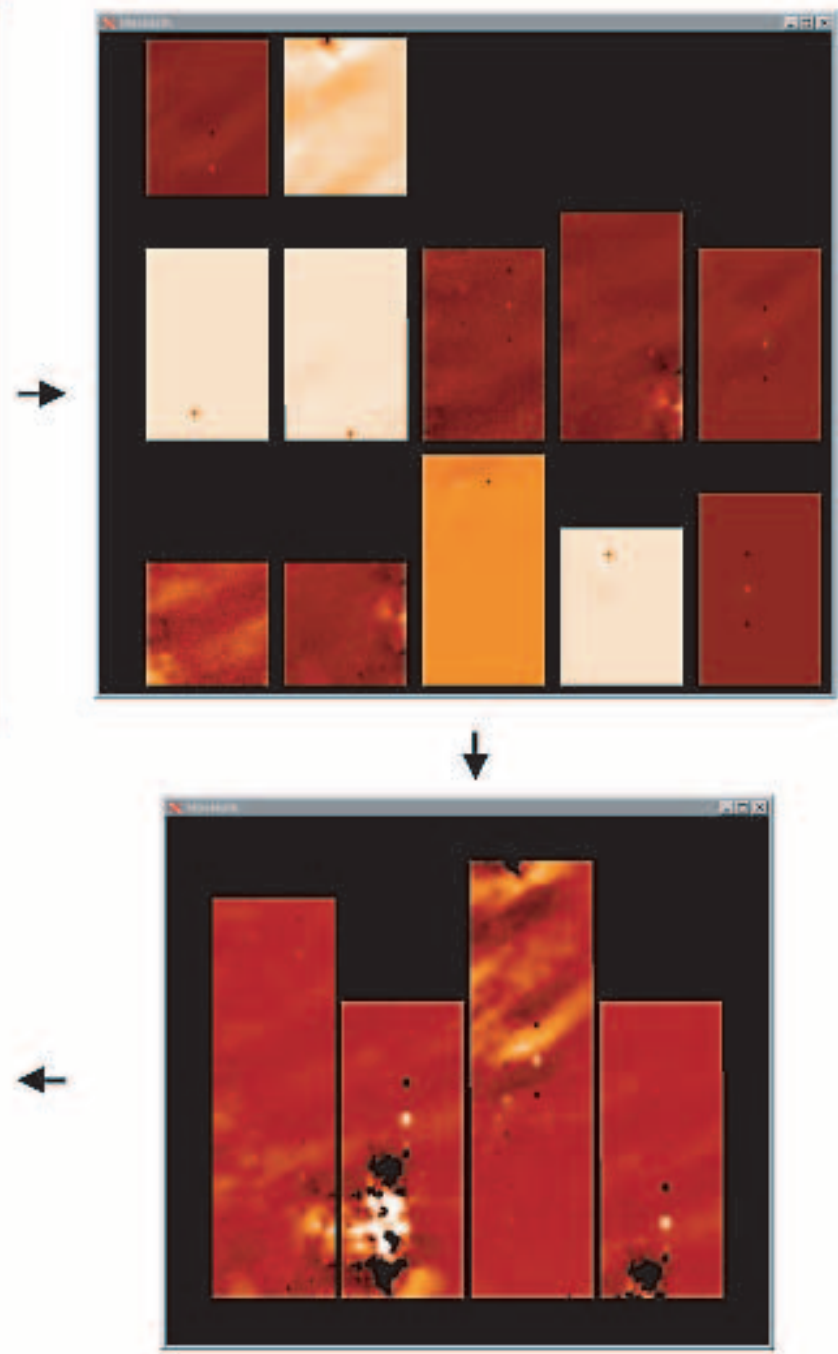

FIG. 1.-Example of the construction of the $10 \mu \mathrm{m}$ mosaic. Clockwise from top left, individual chopped and nodded frames are progressively assembled into a

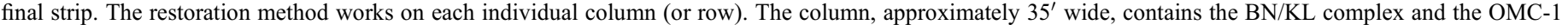
South region. North is at the top and west is on the right.

mosaic, they have been minimized by replacing at their positions the information provided by the reconstructed images obtained with different chopping throws and orientation. Another problem is that the inversion method we have used provides less than ideal results when the signal in the image to be inverted is negative. In this case, the number of iterations needed to recover the signal may be larger than the number of iterations corresponding to optimal noise reduction. Also in this case, these problems have been greatly mitigated at $10 \mu \mathrm{m}$ by using images taken with different orientation and chopping amplitudes.

\subsubsection{Narrowband Images}

The narrowband images were registered using the peak of the $\mathrm{BN}$ object, then combined and trimmed to the same size of 101 pixels $\times 117$ pixels, corresponding to $26^{\prime \prime} .8 \times 31^{\prime \prime} .0$. The astrometric solution was derived assuming for the $\mathrm{BN}$ peak the following coordinates: R.A.(J2000) $=05^{\mathrm{h}} 35^{\mathrm{m}} 14^{\mathrm{s}} 10$ and decl. (J2000) $=-05^{\circ} 22^{\prime} 22^{\prime \prime} .7$, a nominal pixel scale of 0 "' 26 constant across the field, a rotation angle of -0.8 east of north, as measured on the same night for the wide field area project. The BN center was set at the pixel position $(64.2,82.2)$ and measured using a centroid procedure with 5 pixel (1".3) radius.

\subsubsection{Photometry}

Point sources have been identified by visual inspection of the original (chopped and nodded) images and then confirmed on the final reconstructed image. A few sources, indicated in Table 3, appear very faint only in the final mosaic and do not show any counterparts at shorter wavelengths. It is possible that some are artifacts surviving the image cleaning processes described in the previous section, and an independent confirmation is needed to make sure they are real. On the other hand, the variations of exposure time and air mass across the image may have rendered invisible sources otherwise within reach with our instrument in more ideal conditions.

Aperture photometry was obtained on individual chop/nod pairs using circular apertures with radii between 2 and 5 pixels and applying an aperture correction based on the average encircled energy of the standard stars observed during the same night. Typically, all standards were used, with the exception of HR 2061 ( $\alpha$ Ori), known to be extended in the mid-IR (Rinehart et al. 1998) and easily resolved by MAX. An air-mass 
TABLE 3

Mid-Infrared Photometry of Sources in the Orion Nebula

\begin{tabular}{|c|c|c|c|c|c|c|c|c|c|c|}
\hline $\begin{array}{l}\text { MAX } \\
(1)\end{array}$ & $\begin{array}{l}\mathrm{H} 97 \\
(2)\end{array}$ & $\begin{array}{l}\mathrm{HC} 00 \\
(3)\end{array}$ & $\begin{array}{l}\text { MLLA02 } \\
\text { (4) }\end{array}$ & $\begin{array}{l}\text { R.A. (J2000) } \\
\text { (5) }\end{array}$ & $\begin{array}{l}\text { Decl. (J2000) } \\
\quad(6)\end{array}$ & $\begin{array}{c}{[N]} \\
(1998 \mathrm{Nov}) \\
(7)\end{array}$ & $\begin{array}{c}{[N]} \\
(2000 \mathrm{Dec}) \\
(8)\end{array}$ & $\begin{array}{c}{[N]} \\
\text { (mosaic) } \\
(9)\end{array}$ & $\begin{array}{l}{[Q]} \\
(10)\end{array}$ & $\begin{array}{l}\text { Notes } \\
(11)\end{array}$ \\
\hline 1 1........................ & 265 & $\ldots$ & 234 & 053505.52 & -052409.9 & $\ldots$ & $6.55 \pm 0.14$ & $\ldots$ & $\ldots$ & MAX source is $+3 . " 53 \mathrm{E},-0.28 \mathrm{~N}$ \\
\hline $2 \ldots \ldots \ldots \ldots \ldots \ldots \ldots$ & 266 & .. & 223 & 053505.55 & -052414.5 & $\ldots$ & $7.50 \pm 0.39$ & $\ldots$ & $\ldots$ & MAX source is $+3 .^{\prime \prime} 44 \mathrm{E},-0.19 \mathrm{~N}$ \\
\hline $3 \ldots \ldots \ldots \ldots \ldots \ldots \ldots \ldots \ldots$ & 274 & $\ldots$ & 314 & 053505.74 & -052344.5 & $\ldots$ & $6.72 \pm 0.16$ & $\ldots$ & $\ldots$ & MAX source is $+2{ }^{\prime \prime} 10 \mathrm{E}, 0.19 \mathrm{~S}$ \\
\hline 4........................ & 278 & $\ldots$ & 161 & 053505.74 & -052418.4 & $\ldots$ & $5.90 \pm 0.11$ & $\ldots$ & $\ldots$ & MAX source is $+11^{\prime \prime} 55 \mathrm{E}, 0 . " 32 \mathrm{~S}$ \\
\hline $5 \ldots \ldots \ldots \ldots \ldots \ldots \ldots$ & 291 & 99 & 161 & 053506.45 & -052439.0 & $\ldots$ & $\ldots$ & $6.85 \pm 0.15$ & $\ldots$ & \\
\hline 6...………........... & 341 & 305 & 409 & 053510.23 & -052321.0 & $\ldots$ & $4.70 \pm 0.09$ & $\ldots$ & $\ldots$ & \\
\hline 7....................... & $\ldots$ & $\ldots$ & $\ldots$ & 053510.23 & -052143.5 & $\ldots$ & $6.48 \pm 0.18$ & $\ldots$ & $\ldots$ & \\
\hline 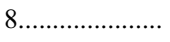 & $\ldots$ & 320 & 425 & 053510.28 & -052315.8 & $\ldots$ & $7.46 \pm 0.14$ & $\ldots$ & $\ldots$ & \\
\hline 9............................ & 349 & 146 & 220 & 053510.56 & -052417.2 & $\ldots$ & $6.78 \pm 0.10$ & $\ldots$ & $\ldots$ & \\
\hline $10 \ldots \ldots \ldots \ldots \ldots \ldots$ & 347 & 554 & 733 & 053510.64 & -052156.8 & $\ldots$ & $6.16 \pm 0.13$ & $\ldots$ & $\ldots$ & \\
\hline $11 \ldots \ldots \ldots \ldots \ldots \ldots$ & 392 & 385 & 507 & 053510.64 & -052255.8 & $\ldots$ & $6.14 \pm 0.09$ & $\ldots$ & $\ldots$ & \\
\hline $12 \ldots \ldots \ldots \ldots \ldots \ldots \ldots$ & 352 & 224 & 316 & 053510.75 & -052344.1 & $\ldots$ & $7.27 \pm 0.13$ & $\ldots$ & $\ldots$ & \\
\hline 13..................... & 355 & 416 & 542 & 053510.90 & -052246.1 & $\ldots$ & $6.37 \pm 0.10$ & $\ldots$ & $\ldots$ & \\
\hline $14 \ldots \ldots \ldots \ldots \ldots \ldots$ & 356 & 93 & 148 & 053510.94 & -052448.5 & $\ldots$ & $6.00 \pm 0.12$ & $\ldots$ & $\ldots$ & \\
\hline $15 \ldots \ldots \ldots \ldots \ldots \ldots$ & $\ldots$ & 415 & 541 & 053511.04 & -052246.3 & $\ldots$ & $6.50 \pm 0.11$ & $\ldots$ & $\ldots$ & \\
\hline $16 \ldots \ldots \ldots \ldots \ldots \ldots$ & $\ldots$ & $\ldots$ & $\ldots$ & 053511.11 & -052153.0 & $\ldots$ & $6.38 \pm 0.17$ & $\ldots$ & $\ldots$ & \\
\hline 17.......................... & $\ldots$ & 434 & 575 & 053511.20 & -052237.6 & $\ldots$ & $5.06 \pm 0.09$ & $\ldots$ & $\ldots$ & \\
\hline 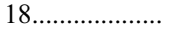 & $\ldots$ & 105 & 171 & 053511.32 & -052438.4 & $\ldots$ & $6.34 \pm 0.13$ & $\ldots$ & $\ldots$ & \\
\hline 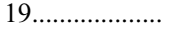 & $\ldots$ & 203 & 289 & 053511.49 & -052351.5 & $\ldots$ & $6.95 \pm 0.10$ & $\ldots$ & $\ldots$ & \\
\hline 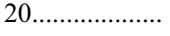 & 366 & 140 & 207 & 053511.65 & -052421.9 & $\ldots$ & $7.99 \pm 0.19$ & & $\cdots$ & \\
\hline 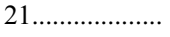 & $\ldots$ & 611 & 809 & 053511.75 & -052131.8 & $\ldots$ & $\ldots$ & $7.23 \pm 0.3$ & $\ldots$ & \\
\hline $22 \ldots \ldots \ldots \ldots \ldots \ldots . .$. & $\ldots$ & 672 & 880 & 053511.88 & -052059.9 & $\ldots$ & $\cdots$ & $5.70 \pm 0.2$ & $\ldots$ & \\
\hline 23........................ & 3058 & 664 & 872 & 053511.92 & -052102.8 & $\ldots$ & $\cdots$ & $6.85 \pm 0.3$ & $\cdots$ & \\
\hline 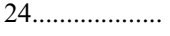 & $\ldots$ & $\ldots$ & 710 & 053511.95 & -052201.5 & $\ldots$ & $\ldots$ & $7.17 \pm 0.4$ & $\ldots$ & \\
\hline $25 \ldots \ldots \ldots \ldots \ldots \ldots$ & 9009 & 395 & 516 & 053511.98 & -052253.6 & $\ldots$ & $6.03 \pm 0.10$ & $\ldots$ & $\ldots$ & \\
\hline 26...................... & 373 & $\ldots$ & 944 & 053512.01 & -052033.8 & $\ldots$ & $\ldots$ & $6.10 \pm 0.3$ & $\ldots$ & \\
\hline 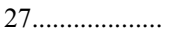 & $378 a, b$ & 215 & $302 \mathrm{~A}, \mathrm{~B}$ & 053512.27 & -052347.8 & $\ldots$ & $6.00 \pm 0.10$ & $\ldots$ & $\ldots$ & \\
\hline $28 \ldots \ldots \ldots \ldots \ldots \ldots . . . .$. & $377 \mathrm{a}, \mathrm{b}$ & 698 & 914A, B & 053512.30 & -052045.6 & $\ldots$ & $\ldots$ & $5.24 \pm 0.3$ & $\ldots$ & \\
\hline $29 \ldots \ldots \ldots \ldots \ldots \ldots \ldots$ & $\ldots$ & 761 & 793 & 053512.41 & -052136.7 & $\ldots$ & $8.08 \pm 0.25$ & $\ldots$ & $\ldots$ & \\
\hline $30 \ldots \ldots \ldots \ldots \ldots \ldots$ & 9018 & 368 & 482 & 053512.58 & -052302.0 & $\ldots$ & $7.43 \pm 0.15$ & $\ldots$ & $\ldots$ & \\
\hline 31......................... & 385 & 228 & 318 & 053512.60 & -052343.9 & $\ldots$ & $5.49 \pm 0.10$ & $\ldots$ & $1.39 \pm 0.20$ & \\
\hline 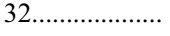 & 391 & 699 & 916 & 053512.85 & -052044.8 & $\ldots$ & .. & $8.97 \pm 0.3$ & $\ldots$ & \\
\hline 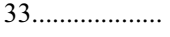 & . & 606,608 & $804 \mathrm{~A}, \mathrm{~B}$ & 053512.85 & -052133.6 & $6.33 \pm 0.13$ & . & $\ldots$ & $\ldots$ & \\
\hline 34....................... & 9029 & 505 & 673 & 053513.07 & -052216.1 & $7.36 \pm 0.31$ & $7.38 \pm 0.29$ & $\ldots$ & $\ldots$ & \\
\hline 35........................ & $399 a, b$ & 487 & $651 \mathrm{~A}, \mathrm{~B}$ & 053513.19 & -052221.8 & $7.27 \pm 0.19$ & $7.04 \pm 0.18$ & $\ldots$ & $\ldots$ & \\
\hline 36........................ & $\cdots$ & $\cdots$ & $\ldots$ & 053513.19 & -052204.6 & $7.14 \pm 0.12$ & $7.35 \pm 0.19$ & $\ldots$ & $\ldots$ & \\
\hline 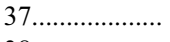 & 401 & 80 & 136 & 053513.20 & -052455.5 & $\ldots$ & $3.79 \pm 0.11$ & $\ldots$ & $\cdots$ & \\
\hline 38........................ & 400 & 682 & 896 & 053513.24 & -052054.7 & $6.34 \pm 0.20$ & $\cdots$ & $\cdots$ & $\cdots$ & \\
\hline 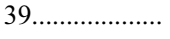 & $\cdots$ & $\ldots$ & $\ldots$ & 053513.31 & -052153.6 & $7.55 \pm 0.26$ & $\cdots$ & $\cdots$ & $\cdots$ & \\
\hline $40 \ldots \ldots \ldots \ldots \ldots \ldots$ & $\ldots$ & 273 & 369 & 053513.40 & -052329.5 & $6.22 \pm 0.09$ & $6.21 \pm 0.11$ & $\ldots$ & $\ldots$ & \\
\hline 41......................... & 409 & 240 & 327 & 053513.44 & -052340.2 & $6.44 \pm 0.09$ & $6.22 \pm 0.10$ & $\ldots$ & $\ldots$ & \\
\hline $42 \ldots \ldots \ldots \ldots \ldots \ldots$ & $\ldots$ & 178 & 263 & 053513.55 & -052400.2 & $6.74 \pm 0.09$ & $6.87 \pm 0.12$ & $\ldots$ & $\ldots$ & \\
\hline 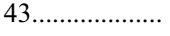 & $\ldots$ & 192 & 276 & 053513.58 & -052355.0 & $6.40 \pm 0.09$ & $6.24 \pm 0.11$ & $\ldots$ & $2.9 \pm 0.3$ & \\
\hline 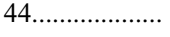 & $\ldots$ & 602 & 797 & 053513.71 & -052135.7 & $6.43 \pm 0.09$ & $6.39 \pm 0.13$ & $\ldots$ & $\cdots$ & \\
\hline 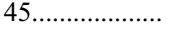 & 424 & 541 & 715 & 053513.79 & -052203.5 & $\cdots$ & $6.28 \pm 0.50$ & $\ldots$ & .. & \\
\hline 46.......................... & 431 & 242 & 328 & 053513.79 & -052340.1 & $2.69 \pm 0.08$ & $2.78 \pm 0.09$ & $\ldots$ & $0.68 \pm 0.19$ & \\
\hline
\end{tabular}


TABLE 3-Continued

\begin{tabular}{|c|c|c|c|c|c|c|c|c|c|c|}
\hline $\begin{array}{l}\text { MAX } \\
\text { (1) }\end{array}$ & $\begin{array}{l}\mathrm{H} 97 \\
(2)\end{array}$ & $\begin{array}{l}\mathrm{HC} 00 \\
(3)\end{array}$ & $\begin{array}{l}\text { MLLA02 } \\
\text { (4) }\end{array}$ & $\begin{array}{l}\text { R.A. (J2000) } \\
\text { (5) }\end{array}$ & $\begin{array}{c}\text { Decl. (J2000) } \\
\text { (6) }\end{array}$ & $\begin{array}{c}{[N]} \\
(1998 \mathrm{Nov}) \\
(7)\end{array}$ & $\begin{array}{c}{[N]} \\
(2000 \mathrm{Dec}) \\
(8)\end{array}$ & $\begin{array}{c}{[N]} \\
\text { (mosaic) } \\
(9)\end{array}$ & $\begin{array}{l}{[Q]} \\
(10)\end{array}$ & $\begin{array}{l}\text { Notes } \\
(11)\end{array}$ \\
\hline 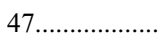 & $\ldots$ & $\ldots$ & $\ldots$ & $\begin{array}{lll}05 & 35 & 13.8\end{array}$ & -052357.0 & $\ldots$ & $\ldots$ & $\ldots$ & $1.55 \pm 0.18$ & \\
\hline 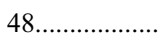 & 423 & 703 & 703 & 053513.81 & -052207.8 & $4.90 \pm 0.08$ & $4.70 \pm 0.09$ & $\ldots$ & $\ldots$ & \\
\hline 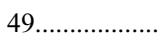 & $\ldots$ & 548 & 726 & 053513.81 & -052200.6 & $1.98 \pm 0.08$ & $1.90 \pm 0.09$ & $\ldots$ & $-0.64 \pm 0.16$ & \\
\hline 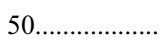 & & & & 053513.9 & $-05 \quad 22 \quad 12.0$ & $\ldots$ & $\ldots$ & $\ldots$ & $0.98 \pm 0.22$ & \\
\hline $51 \ldots \ldots \ldots \ldots \ldots \ldots$ & 9053 & 629 & 828 & 053513.96 & -052122.8 & $5.99 \pm 0.08$ & $\ldots$ & $\ldots$ & $\ldots$ & \\
\hline $52 \ldots \ldots \ldots \ldots \ldots \ldots$ & $\ldots$ & 314 & 413 & 053513.97 & -052320.1 & $\ldots$ & $\ldots$ & $6.73 \pm 0.3$ & $\ldots$ & \\
\hline $53 \ldots \ldots \ldots \ldots \ldots \ldots$ & $\ldots$ & 134 & 199 & 053514.01 & -052425.4 & $\ldots$ & $7.47 \pm 0.21$ & $\ldots$ & $\ldots$ & \\
\hline $54 \ldots \ldots \ldots \ldots \ldots \ldots$ & $\ldots$ & $\ldots$ & $\ldots$ & 053514.1 & -052300.0 & $\ldots$ & $\ldots$ & $\ldots$ & $1.65 \pm 0.21$ & \\
\hline $55 \ldots \ldots \ldots \ldots . . . . . .$. & $\ldots$ & 704 & 707 & 053514.12 & -052205.4 & $6.78 \pm 0.50$ & $\ldots$ & $\ldots$ & $\ldots$ & \\
\hline $56 \ldots \ldots \ldots \ldots \ldots$ & & & & 053514.18 & -052314.8 & $\ldots$ & $5.99 \pm 0.21$ & $\ldots$ & $\ldots$ & \\
\hline 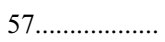 & 437 & 135 & 203 & 053514.29 & -052424.7 & $5.57 \pm 0.09$ & $5.66 \pm 0.10$ & $\ldots$ & $\ldots$ & \\
\hline $58 \ldots \ldots \ldots \ldots \ldots$ & $\ldots$ & 361 & 476 & 053514.30 & -052304.3 & $6.04 \pm 0.09$ & $6.51 \pm 0.11$ & $\ldots$ & $\ldots$ & \\
\hline 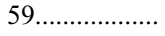 & 9061 & 345 & 452 & 053514.31 & -052308.4 & $5.81 \pm 0.08$ & $5.51 \pm 0.10$ & $\ldots$ & $\ldots$ & \\
\hline $60 \ldots \ldots \ldots \ldots \ldots$ & 9062 & 399 & 518 & 053514.33 & -052253.7 & $6.16 \pm 0.09$ & $6.18 \pm 0.14$ & $\ldots$ & $\ldots$ & \\
\hline $61 \ldots \ldots \ldots \ldots \ldots \ldots$ & $\ldots$ & $\ldots$ & 292 & 053514.38 & -052350.6 & $4.40 \pm 0.08$ & $3.68 \pm 0.09$ & $\ldots$ & $0.42 \pm 0.18$ & \\
\hline $62 \ldots \ldots \ldots \ldots \ldots \ldots$ & 441 & 258 & 350 & 053514.40 & -052333.8 & $5.83 \pm 0.08$ & $6.05 \pm 0.11$ & $\ldots$ & $\ldots$ & \\
\hline $63 \ldots \ldots \ldots \ldots \ldots \ldots$ & 9069 & 369 & 485 & 053514.58 & -052302.6 & $\ldots$ & $6.97 \pm 0.19$ & $\ldots$ & $\ldots$ & MAX source is $-2.180 \mathrm{E},+0.29 \mathrm{~S}$ \\
\hline 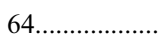 & $\ldots$ & 189,193 & $273 \mathrm{~A}, \mathrm{~B}$ & 053514.54 & -052355.7 & $5.44 \pm 0.08$ & $5.27 \pm 0.09$ & $\ldots$ & $1.91 \pm 0.22$ & \\
\hline 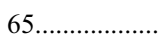 & $\ldots$ & 411 & 534 & 053514.63 & -052250.2 & $\ldots$ & $6.95 \pm 0.19$ & $\ldots$ & $\ldots$ & MAX source is $-2 . " 25 \mathrm{E},-0.52 \mathrm{~N}$ \\
\hline 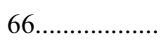 & 9073,9075 & 714 & 467A, B & 053514.84 & -052305.0 & $6.58 \pm 0.19$ & $\ldots$ & $\ldots$ & $\ldots$ & MAX source is $-3 . " 35 \mathrm{E}, 0.24 \mathrm{~N}$ \\
\hline 67...................... & $445 \mathrm{a}, \mathrm{b}$ & & 922A, B & 053514.69 & -052043.7 & $\ldots$ & $\ldots$ & $6.60 \pm 0.3$ & $\ldots$ & \\
\hline 68....................... & 9071 & 657 & 863 & 053514.71 & -052105.9 & $6.32 \pm 0.10$ & $\ldots$ & $\ldots$ & $\ldots$ & \\
\hline 69..................... & 454 & 431 & 568 & 053514.91 & -052239.6 & $4.79 \pm 0.08$ & $4.75 \pm 0.10$ & $\ldots$ & $\ldots$ & \\
\hline 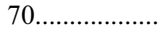 & $\ldots$ & 437 & 580 & 053515.19 & -052237.3 & $6.59 \pm 0.10$ & $6.53 \pm 0.26$ & $\ldots$ & $\ldots$ & \\
\hline 71....................... & 463 & 398 & 514 & $0535 \quad 15.21$ & -052254.7 & $4.44 \pm 0.08$ & $4.28 \pm 0.10$ & $\ldots$ & $\ldots$ & \\
\hline $72 \ldots \ldots \ldots \ldots \ldots$ & $\ldots$ & $\ldots$ & $\ldots$ & 053515.34 & -052250.4 & $7.21 \pm 0.17$ & $\ldots$ & $\ldots$ & $\ldots$ & \\
\hline $73 \ldots \ldots \ldots \ldots$ & 470 & 504 & 671 & $0535 \quad 15.34$ & -052216.3 & $4.85 \pm 0.08$ & $4.60 \pm 0.10$ & $\ldots$ & $\ldots$ & \\
\hline $74 \ldots \ldots \ldots \ldots \ldots \ldots$ & $472 \mathrm{a}, \mathrm{b}, 9096$ & 475,476 & $630 \mathrm{~A}, \mathrm{~B}, \mathrm{C}$ & 053515.35 & -052225.7 & $7.00 \pm 0.11$ & $7.07 \pm 0.32$ & $\ldots$ & $\ldots$ & \\
\hline $75 \ldots \ldots \ldots \ldots \ldots \ldots$ & 473 & 646 & 848 & 053515.40 & -052113.6 & $5.45 \pm 0.08$ & $\ldots$ & $\ldots$ & $\ldots$ & \\
\hline 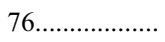 & 475 & 223 & 311 & 053515.43 & -052345.0 & $6.81 \pm 0.09$ & $7.26 \pm 0.16$ & $\ldots$ & $\ldots$ & \\
\hline 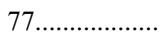 & $\ldots$ & $\ldots$ & $\ldots$ & 053515.49 & -052352.3 & $7.31 \pm 0.17$ & $\ldots$ & $\ldots$ & $\ldots$ & \\
\hline 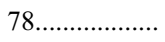 & $\ldots$ & $\ldots$ & $\ldots$ & 053515.50 & -052348.2 & $\ldots$ & $6.21 \pm 0.11$ & $\ldots$ & $\ldots$ & \\
\hline 79..................... & 5178 & 251 & 340 & $0535 \quad 15.52$ & -052337.5 & $6.51 \pm 0.09$ & $5.97 \pm 0.12$ & $\ldots$ & $1.39 \pm 0.20$ & \\
\hline $80 \ldots \ldots \ldots \ldots \ldots \ldots$ & & & & 053515.6 & -052310.3 & $\ldots$ & $\ldots$ & $\ldots$ & $1.59 \pm 0.21$ & \\
\hline $81 \ldots \ldots \ldots \ldots \ldots$ & 480 & 172 & 249 & 053515.64 & -052403.9 & $6.26 \pm 0.09$ & $6.49 \pm 0.11$ & $\ldots$ & $\ldots$ & \\
\hline $82 \ldots \ldots \ldots \ldots \ldots$ & 479 & 389 & 503 & 053515.65 & -052256.7 & $3.93 \pm 0.08$ & $3.87 \pm 0.09$ & $\ldots$ & $\ldots$ & \\
\hline 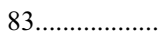 & $488 w$ & 307 & 405 & 053515.72 & -052322.7 & $5.75 \pm 0.09$ & $5.47 \pm 0.11$ & $\ldots$ & $1.41 \pm 0.24^{\mathrm{a}}$ & \\
\hline 84......................... & 492 & 420 & 545 & 053515.78 & -052246.4 & $8.33 \pm 0.82$ & $\ldots$ & $\ldots$ & $\ldots$ & \\
\hline 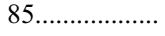 & 1865 & 336 & 440 & 053515.80 & -052314.6 & $5.43 \pm 0.25$ & $\ldots$ & $\ldots$ & $\ldots$ & \\
\hline $86 \ldots \ldots \ldots \ldots \ldots \ldots$ & 489 & 287 & 381 & 053515.80 & -052326.7 & $5.44 \pm 0.08$ & $5.28 \pm 0.11$ & $\ldots$ & $\ldots$ & \\
\hline $87 \ldots \ldots \ldots \ldots \ldots \ldots . .$. & $488 \mathrm{a}$ & 306 & 404 & 053515.86 & -052322.5 & $4.48 \pm 0.08$ & $4.46 \pm 0.11$ & $\ldots$ & $1.41 \pm 0.24^{\mathrm{a}}$ & \\
\hline 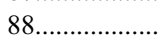 & $\ldots$ & 291 & 385 & 053515.86 & -052325.0 & $5.65 \pm 0.18$ & $\ldots$ & $\ldots$ & $\ldots$ & OW $158-326^{\mathrm{b}}$ \\
\hline 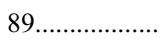 & 490 & $\ldots$ & 925 & 053515.88 & -052039.7 & $\ldots$ & $\ldots$ & $8.83 \pm 0.3$ & $\ldots$ & \\
\hline 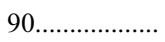 & 9118 & 145 & 213 & 053515.90 & -052418.0 & $\ldots$ & $6.91 \pm 0.12$ & $\ldots$ & $\ldots$ & \\
\hline $91 \ldots \ldots \ldots \ldots \ldots$ & 496 & 490 & 653 & 053515.93 & -052221.8 & $6.26 \pm 0.09$ & $\ldots$ & $\ldots$ & $\ldots$ & \\
\hline $92 \ldots \ldots \ldots \ldots \ldots$ & 499 & 213 & 296 & 053515.95 & -052349.9 & $3.18 \pm 0.08$ & $3.40 \pm 0.09$ & $\ldots$ & $1.42 \pm 0.19$ & \\
\hline 93................... & & & & 053515.97 & -052236.1 & $6.86 \pm 0.22$ & $\ldots$ & $\ldots$ & $\ldots$ & \\
\hline 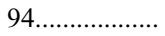 & 503 & 202 & 288 & 053516.01 & -052352.8 & $5.16 \pm 0.08$ & $5.08 \pm 0.10$ & $\ldots$ & $\ldots$ & \\
\hline
\end{tabular}


TABLE 3-Continued

\begin{tabular}{|c|c|c|c|c|c|c|c|c|c|c|}
\hline $\begin{array}{l}\text { MAX } \\
\text { (1) }\end{array}$ & $\begin{array}{l}\mathrm{H} 97 \\
(2)\end{array}$ & $\begin{array}{l}\mathrm{HC} 00 \\
(3)\end{array}$ & $\begin{array}{l}\text { MLLA02 } \\
\text { (4) }\end{array}$ & $\begin{array}{l}\text { R.A. (J2000) } \\
\quad(5)\end{array}$ & $\begin{array}{c}\text { Decl. (J2000) } \\
\text { (6) }\end{array}$ & $\begin{array}{c}{[N]} \\
(1998 \text { Nov) } \\
(7)\end{array}$ & $\begin{array}{c}{[N]} \\
(2000 \mathrm{Dec}) \\
(8)\end{array}$ & $\begin{array}{c}{[N]} \\
\text { (mosaic) } \\
(9)\end{array}$ & $\begin{array}{l}{[Q]} \\
(10)\end{array}$ & $\begin{array}{l}\text { Notes } \\
(11)\end{array}$ \\
\hline $95 \ldots \ldots \ldots \ldots \ldots \ldots$ & & & & 053516.05 & -052233.4 & $6.68 \pm 0.10$ & & $\ldots$ & $\ldots$ & \\
\hline $96 \ldots \ldots \ldots \ldots \ldots . .$. & 9128 & 393 & 511 & 053516.07 & -052254.4 & $5.50 \pm 0.08$ & $5.07 \pm 0.10$ & $\ldots$ & $\ldots$ & \\
\hline $97 \ldots \ldots \ldots \ldots \ldots . .$. & $1863 b$ & 350 & $460 \mathrm{~B}$ & 053516.08 & -052307.2 & $3.63 \pm 0.09$ & $3.66 \pm 0.09$ & $\ldots$ & $1.44 \pm 0.19$ & \\
\hline $98 \ldots \ldots \ldots \ldots \ldots . .$. & $\ldots$ & 296 & 389 & 053516.09 & -052324.2 & $3.93 \pm 0.08$ & $3.98 \pm 0.10$ & $\ldots$ & $1.18 \pm 0.19$ & \\
\hline $99 \ldots \ldots \ldots \ldots \ldots \ldots . .$. & $\ldots$ & $504 \mathrm{a}, \mathrm{b}$ & 926 & 053516.13 & -052038.4 & $\ldots$ & $\ldots$ & $8.13 \pm 0.3$ & $\ldots$ & \\
\hline $100 \ldots \ldots \ldots \ldots \ldots \ldots$ & $\ldots$ & $\ldots$ & $\ldots$ & 053516.15 & -052226.2 & $6.86 \pm 0.19$ & $\ldots$ & $\ldots$ & $\ldots$ & To be confirmed \\
\hline $101 \ldots \ldots \ldots \ldots \ldots \ldots$ & $1863 a$ & 354 & $460 \mathrm{~A}$ & 053516.15 & $\begin{array}{lll}-05 & 23 & 07.1\end{array}$ & $5.76 \pm 0.37$ & $6.52 \pm 0.10$ & $\ldots$ & $\ldots$ & \\
\hline $102 \ldots \ldots \ldots \ldots \ldots \ldots$ & $511 \mathrm{a}, \mathrm{b}$ & 522 & 689A, B & 053516.28 & $\begin{array}{lll}-05 & 22 & 10.5\end{array}$ & $5.63 \pm 0.08$ & $5.65 \pm 0.09$ & $\ldots$ & $\ldots$ & \\
\hline 103..................... & 9135 & 652 & 857 & $\begin{array}{lll}05 & 35 & 16.28\end{array}$ & -052108.9 & $6.40 \pm 0.10$ & $\ldots$ & $\ldots$ & $\ldots$ & \\
\hline $104 \ldots \ldots \ldots \ldots \ldots \ldots$ & 513 & 412 & 535 & 053516.31 & -052249.0 & $6.62 \pm 0.33$ & $6.97 \pm 0.19$ & $\ldots$ & $\ldots$ & \\
\hline $105 \ldots \ldots \ldots \ldots \ldots \ldots$ & 512 & 322 & 422 & 053516.32 & -052316.7 & $4.24 \pm 0.08$ & $4.35 \pm 0.09$ & $\ldots$ & $1.06 \pm 0.26$ & \\
\hline $106 \ldots \ldots \ldots \ldots \ldots \ldots$ & $\ldots$ & $\ldots$ & 401B & 053516.34 & -052322.6 & $3.06 \pm 0.08$ & $3.07 \pm 0.09$ & $\ldots$ & $0.27 \pm 0.17$ & $\mathrm{SC} 3^{\mathrm{c}}$ \\
\hline 107....................... & $\ldots$ & $\ldots$ & $\ldots$ & 053516.4 & -052256.0 & $\ldots$ & $\ldots$ & $\ldots$ & $1.24 \pm 0.20$ & \\
\hline $108 \ldots \ldots \ldots \ldots \ldots \ldots$ & 515 & 171 & 246 & 053516.41 & -052404.2 & $4.94 \pm 0.08$ & $5.15 \pm 0.09$ & $\ldots$ & $\ldots$ & \\
\hline $109 \ldots \ldots \ldots \ldots \ldots \ldots$ & $\ldots$ & 112 & 173 & 053516.41 & -052436.9 & $\cdots$ & $\ldots$ & $8.10 \pm 0.3$ & $\ldots$ & \\
\hline $110 \ldots \ldots \ldots \ldots \ldots \ldots$ & $\ldots$ & 514 & 682 & 053516.42 & -052213.0 & $5.52 \pm 0.08$ & $4.88 \pm 0.09$ & $\ldots$ & $\ldots$ & \\
\hline $111 \ldots \ldots \ldots \ldots \ldots \ldots$ & 1891 & 309 & $401 \mathrm{~A}$ & 053516.46 & -052322.7 & $4.23 \pm 0.08$ & $4.62 \pm 0.09$ & $\ldots$ & $\ldots$ & \\
\hline $112 \ldots \ldots \ldots \ldots \ldots \ldots \ldots$ & $\ldots$ & $\ldots$ & $\ldots$ & 053516.51 & -052248.0 & $\cdots$ & $6.79 \pm 0.65$ & $\ldots$ & $\ldots$ & \\
\hline $113 \ldots \ldots \ldots \ldots \ldots \ldots$ & $\ldots$ & $\ldots$ & $\ldots$ & 053516.51 & $-0523 \quad 30.2$ & $6.07 \pm 0.31$ & $\cdots$ & $\cdots$ & $\cdots$ & To be confirmed \\
\hline $114 \ldots \ldots \ldots \ldots \ldots \ldots$ & $\ldots$ & 325 & 426 & 053516.52 & -052315.5 & $5.45 \pm 0.25$ & $\ldots$ & $\ldots$ & & \\
\hline $115 \ldots \ldots \ldots \ldots \ldots \ldots$ & & 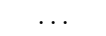 & & 053516.7 & -052257.0 & & & $\ldots$ & $1.12 \pm 0.21$ & \\
\hline $116 \ldots \ldots \ldots \ldots \ldots \ldots$ & 524 & 323 & 424 & 053516.76 & -052316.5 & $3.86 \pm 0.08$ & $4.26 \pm 0.09$ & $\ldots$ & $0.46 \pm 0.23$ & \\
\hline $117 \ldots \ldots \ldots \ldots \ldots \ldots$ & $\ldots$ & $\ldots$ & $\ldots$ & 053516.82 & -052358.2 & $\ldots$ & $5.97 \pm 0.74$ & $\ldots$ & $\ldots$ & \\
\hline $118 \ldots \ldots \ldots \ldots \ldots$ & $\ldots$ & 289 & 383A, B & 053516.84 & -052326.0 & $2.15 \pm 0.08$ & $2.29 \pm 0.10$ & $\cdots$ & $-0.64 \pm 0.18$ & \\
\hline 119..................... & 532 & 414 & 537 & 053516.98 & -052249.1 & $\ldots$ & $6.95 \pm 0.21$ & $\ldots$ & $\ldots$ & \\
\hline $120 \ldots \ldots \ldots \ldots \ldots$ & $\ldots$ & $\ldots$ & $\ldots$ & 053516.98 & -052231.0 & .. & $6.97 \pm 0.42$ & $\ldots$ & $\ldots$ & \\
\hline $121 \ldots \ldots \ldots \ldots \ldots$ & 534 & 252 & 341 & 053516.99 & -052337.1 & $4.90 \pm 0.08$ & $5.40 \pm 0.09$ & $\cdots$ & $0.89 \pm 0.21$ & \\
\hline $122 \ldots \ldots \ldots \ldots \ldots \ldots$ & 533 & 375 & 489 & 053516.99 & $\begin{array}{lll}-05 & 23 & 01.3\end{array}$ & $6.32 \pm 0.09$ & $5.94 \pm 0.12$ & $\ldots$ & $\ldots$ & \\
\hline $123 \ldots \ldots \ldots \ldots \ldots \ldots$ & 537 & 244 & 330 & 053517.03 & -052340.1 & $\ldots$ & $6.65 \pm 0.19$ & $\ldots$ & $\ldots$ & \\
\hline $124 \ldots \ldots \ldots \ldots \ldots$ & 538 & 259 & 348 & 053517.06 & -052334.0 & $4.45 \pm 0.08$ & $4.24 \pm 0.10$ & $\ldots$ & $\ldots$ & \\
\hline $125 \ldots \ldots \ldots \ldots \ldots$ & $\ldots$ & $\ldots$ & $\ldots$ & 053517.11 & -052326.6 & $\ldots$ & $5.09 \pm 0.12$ & $\ldots$ & $0.78 \pm 0.25$ & \\
\hline $126 \ldots \ldots \ldots \ldots \ldots$ & $\ldots$ & $\ldots$ & $\ldots$ & 053517.11 & $\begin{array}{lll}-05 & 23 & 29.3\end{array}$ & $\ldots$ & $5.02 \pm 0.11$ & $\ldots$ & $\ldots$ & To be confirmed \\
\hline 127.................... & 1889 & $\ldots$ & $423 \mathrm{~A}, \mathrm{~B}$ & 053517.26 & $\begin{array}{lll}-05 & 23 & 17.0\end{array}$ & & $5.97 \pm 0.46$ & $\ldots$ & $\ldots$ & \\
\hline $128 \ldots \ldots \ldots \ldots \ldots \ldots$ & 548 & 440 & 584 & 053517.37 & -052235.6 & $5.52 \pm 0.08$ & $5.43 \pm 0.10$ & $\ldots$ & $\ldots$ & \\
\hline $129 \ldots \ldots \ldots \ldots \ldots \ldots \ldots$ & 549 & 180 & 261 & 053517.39 & -052400.1 & $6.03 \pm 0.08$ & $6.00 \pm 0.10$ & $\ldots$ & $\ldots$ & \\
\hline $130 \ldots \ldots \ldots \ldots \ldots \ldots \ldots$ & 9180 & 313 & 411 & 053517.46 & -052321.1 & $5.41 \pm 0.09$ & $4.89 \pm 0.34$ & $\ldots$ & $\cdots$ & \\
\hline 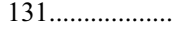 & 554 & 295 & 387 & 053517.55 & -052325.0 & $5.94 \pm 0.09$ & $5.92 \pm 0.11$ & $\ldots$ & $1.7 \pm 0.3$ & \\
\hline $132 \ldots \ldots \ldots \ldots \ldots \ldots$ & $553 \mathrm{a}, \mathrm{b}$ & 388 & $499.2 \mathrm{~A}, \mathrm{~B}^{\mathrm{d}}$ & 053517.57 & -052257.10 & $5.67 \pm 0.09$ & $5.72 \pm 0.10$ & $\ldots$ & $\ldots$ & \\
\hline $133 \ldots \ldots \ldots \ldots \ldots \ldots$ & $552 \mathrm{a}, \mathrm{b}$ & 586 & 773A, B & 053517.57 & -052145.9 & $5.30 \pm 0.08$ & $5.15 \pm 0.11$ & $\ldots$ & $\ldots$ & \\
\hline $134 \ldots \ldots \ldots \ldots \ldots \ldots$ & $\ldots$ & 563 & 743 & 053517.62 & -052154.3 & $6.33 \pm 0.09$ & $6.69 \pm 0.17$ & $\ldots$ & $\ldots$ & \\
\hline $135 \ldots \ldots \ldots \ldots \ldots \ldots$ & 558 & 241 & $325 \mathrm{~A}$ & 053517.68 & -052341.1 & $6.44 \pm 0.10$ & $\ldots$ & $\ldots$ & $\ldots$ & \\
\hline $136 \ldots \ldots \ldots \ldots \ldots \ldots$ & 9195 & 234 & 320 & 053517.75 & -052342.7 & $6.88 \pm 0.11$ & $\cdots$ & $\ldots$ & $\cdots$ & \\
\hline $137 \ldots \ldots \ldots \ldots \ldots \ldots$ & 561 & 681 & 892 & 053517.82 & -052054.2 & $\cdots$ & $\cdots$ & $7.77 \pm 0.3$ & $\ldots$ & \\
\hline 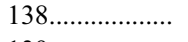 & 9201 & 367 & 480 & 053517.90 & $\begin{array}{lll}-05 & 23 & 02.0\end{array}$ & $5.91 \pm 0.32$ & $\cdots$ & $\cdots$ & $\cdots$ & \\
\hline $139 \ldots \ldots \ldots \ldots \ldots$ & $\ldots$ & 542 & 714 & 053517.90 & $\begin{array}{ll}-05 & 2203.4\end{array}$ & $6.16 \pm 0.09$ & $6.07 \pm 0.11$ & $\ldots$ & $\ldots$ & \\
\hline $140 \ldots \ldots \ldots \ldots \ldots \ldots$ & 567 & 425 & 549 & 053517.92 & -052244.6 & $6.13 \pm 0.09$ & $6.27 \pm 0.11$ & $\ldots$ & $\ldots$ & \\
\hline $141 \ldots \ldots \ldots \ldots \ldots$ & 9196 & 230 & 317 & 053517.93 & -052344.5 & $7.46 \pm 0.34$ & $\ldots$ & $\cdots$ & $\cdots$ & \\
\hline
\end{tabular}


TABLE 3-Continued

\begin{tabular}{|c|c|c|c|c|c|c|c|c|c|c|}
\hline $\begin{array}{l}\text { MAX } \\
\text { (1) }\end{array}$ & $\begin{array}{l}\mathrm{H} 97 \\
(2)\end{array}$ & $\begin{array}{l}\mathrm{HC} 00 \\
(3)\end{array}$ & $\begin{array}{l}\text { MLLA02 } \\
\text { (4) }\end{array}$ & $\begin{array}{l}\text { R.A. (J2000) } \\
\qquad(5)\end{array}$ & $\begin{array}{c}\text { Decl. (J2000) } \\
\text { (6) }\end{array}$ & $\begin{array}{c}{[N]} \\
(1998 \mathrm{Nov}) \\
(7)\end{array}$ & $\begin{array}{c}{[N]} \\
(2000 \mathrm{Dec}) \\
(8)\end{array}$ & $\begin{array}{c}{[N]} \\
\text { (mosaic) } \\
(9)\end{array}$ & $\begin{array}{l}{[Q]} \\
(10)\end{array}$ & $\begin{array}{c}\text { Notes } \\
(11)\end{array}$ \\
\hline 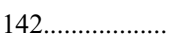 & & 679 & 890 & 053517.96 & -052055.8 & $\ldots$ & & $6.34 \pm 0.3$ & $\ldots$ & \\
\hline $143 \ldots \ldots \ldots \ldots \ldots$ & 9210 & 174 & 248 & 053518.04 & -052403.0 & $4.86 \pm 0.08$ & $4.76 \pm 0.10$ & $\ldots$ & $\ldots$ & \\
\hline 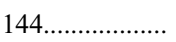 & 575 & 177 & 256 & 053518.08 & -052401.2 & $6.43 \pm 0.12$ & $6.53 \pm 0.10$ & $\ldots$ & $\ldots$ & \\
\hline $145 \ldots \ldots \ldots \ldots \ldots \ldots$ & MS-107 & 372 & 486 & 053518.11 & -052302.8 & $6.71 \pm 0.23$ & $\ldots$ & $\ldots$ & $\ldots$ & \\
\hline $146 \ldots \ldots \ldots \ldots \ldots \ldots$ & 581 & 253 & 344 & 053518.21 & -052336.0 & $4.00 \pm 0.08$ & $4.44 \pm 0.09$ & $\ldots$ & $\ldots$ & \\
\hline $147 \ldots \ldots \ldots \ldots \ldots \ldots$ & $\ldots$ & 533 & 705 & $0535 \quad 18.24$ & -052205.7 & $7.18 \pm 0.15$ & $7.05 \pm 0.17$ & $\ldots$ & $\ldots$ & \\
\hline $148 \ldots \ldots \ldots \ldots \ldots \ldots$ & $\ldots$ & $\ldots$ & & 053518.31 & -052222.6 & $7.38 \pm 0.16$ & $\ldots$ & $\ldots$ & $\ldots$ & \\
\hline 149 & 9220 & 348 & 455 & 053518.36 & -052306.7 & $6.71 \pm 0.17$ & $\ldots$ & $\ldots$ & $\ldots$ & \\
\hline $150 \ldots \ldots \ldots \ldots \ldots$ & 582 & 331 & 431 & 053518.37 & -052315.7 & $6.93 \pm 0.20$ & $\ldots$ & $\ldots$ & $\ldots$ & \\
\hline $151 \ldots \ldots \ldots \ldots \ldots$ & $\ldots$ & $\ldots$ & $\ldots$ & 053518.65 & -052343.1 & $\ldots$ & $7.85 \pm 0.17$ & $\ldots$ & $\ldots$ & \\
\hline $152 \ldots \ldots \ldots \ldots \ldots \ldots$ & 596 & 337 & 441 & 053518.65 & -052313.9 & $3.28 \pm 0.08$ & $3.45 \pm 0.09$ & $\ldots$ & $\ldots$ & \\
\hline 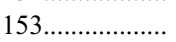 & $598 \mathrm{a}, \mathrm{b}$ & 713 & $499.1 \mathrm{~A}, \mathrm{~B}^{\mathrm{d}}$ & 053518.70 & -052256.9 & $5.16 \pm 0.08$ & $5.37 \pm 0.11$ & $\ldots$ & $\ldots$ & \\
\hline $154 \ldots \ldots \ldots \ldots \ldots \ldots$ & 607 & 214 & 298 & 053519.04 & -052349.6 & $\ldots$ & $3.91 \pm 0.09$ & $\ldots$ & $\ldots$ & \\
\hline $155 \ldots \ldots \ldots \ldots \ldots \ldots$ & $\ldots$ & 351 & $\ldots$ & 053519.6 & -052307.0 & $\ldots$ & $\ldots$ & $\ldots$ & $2.8 \pm 0.25$ & \\
\hline $156 \ldots \ldots \ldots \ldots \ldots \ldots$ & 622 & 133 & 193 & 053519.67 & -052426.5 & $\ldots$ & $5.71 \pm 0.10$ & $\ldots$ & $\ldots$ & \\
\hline $157 \ldots \ldots \ldots \ldots \ldots \ldots$ & 624 & 492 & 648 & 053519.84 & -052221.9 & $\ldots$ & $6.32 \pm 0.10$ & $\ldots$ & $\ldots$ & \\
\hline $158 \ldots \ldots \ldots \ldots \ldots \ldots$ & 3075 & 346 & 450 & 053520.31 & -052306.9 & $\ldots$ & $\ldots$ & $7.57 \pm 0.3$ & $\ldots$ & \\
\hline 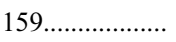 & $648 \mathrm{a}, \mathrm{b}$ & 277,279 & $366 \mathrm{~A}, \mathrm{~B}$ & 053520.45 & -052329.9 & $\ldots$ & $6.21 \pm 0.10$ & $\ldots$ & $\ldots$ & \\
\hline $160 \ldots \ldots \ldots \ldots \ldots \ldots$ & 9266 & 308 & 398 & 053520.48 & -052322.9 & $\ldots$ & $7.60 \pm 0.18$ & $\ldots$ & $\ldots$ & \\
\hline 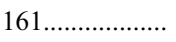 & 652 & 141 & 208 & 053520.56 & -052420.9 & $\ldots$ & $7.37 \pm 0.14$ & $\ldots$ & $\ldots$ & \\
\hline $162 \ldots \ldots \ldots \ldots \ldots$ & 658 & 97 & 154 & 053520.62 & -052446.1 & $\ldots$ & $5.78 \pm 0.17$ & $\ldots$ & $\ldots$ & \\
\hline $163 \ldots \ldots \ldots \ldots \ldots$ & 660 & 590 & 777 & 053520.74 & -052145.2 & $\ldots$ & $6.23 \pm 0.13$ & $\ldots$ & $\ldots$ & \\
\hline $164 \ldots \ldots \ldots \ldots \ldots \ldots$ & 661 & 560 & 737 & 053520.80 & -052156.1 & $\ldots$ & $6.64 \pm 0.14$ & $\ldots$ & $\ldots$ & \\
\hline $165 \ldots \ldots \ldots \ldots \ldots \ldots$ & 665 & 574 & 753 & 053520.95 & -052151.5 & $\ldots$ & $6.02 \pm 0.12$ & $\ldots$ & $\ldots$ & \\
\hline $166 \ldots \ldots \ldots \ldots \ldots \ldots$ & 669 & 217 & 300 & 053521.02 & -052349.2 & $\ldots$ & $7.51 \pm 0.18$ & $\ldots$ & $\ldots$ & \\
\hline $167 \ldots \ldots \ldots \ldots \ldots \ldots$ & $\ldots$ & $\ldots$ & $\ldots$ & 053521.33 & -052342.7 & $\ldots$ & $6.86 \pm 0.13$ & $\ldots$ & $\ldots$ & \\
\hline $168 \ldots \ldots \ldots \ldots \ldots \ldots$ & $687 \mathrm{a}, \mathrm{b}$ & 585,255 & 769A, B & 053521.67 & -052148.1 & $\ldots$ & $5.86 \pm 0.13$ & $\ldots$ & $\ldots$ & \\
\hline $169 \ldots \ldots \ldots \ldots \ldots \ldots$ & 694 & 249 & 332 & 053521.78 & -052339.5 & $\ldots$ & $7.12 \pm 0.12$ & $\ldots$ & $\ldots$ & \\
\hline $170 \ldots \ldots \ldots \ldots \ldots \ldots$ & 698 & 204 & 282 & 053521.79 & -052353.8 & $\ldots$ & $7.57 \pm 0.21$ & $\ldots$ & $\ldots$ & \\
\hline 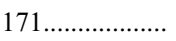 & 9294 & 358 & 465 & 053521.84 & -052306.9 & $\ldots$ & $\ldots$ & $8.01 \pm 0.3$ & $\ldots$ & \\
\hline $172 \ldots \ldots \ldots \ldots \ldots \ldots$ & $\ldots$ & $\ldots$ & $\ldots$ & 053521.94 & -052228.9 & $\ldots$ & $7.28 \pm 0.42$ & $\ldots$ & $\ldots$ & \\
\hline 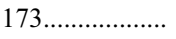 & 5179 & 121 & 182 & 053522.10 & -052432.9 & $\ldots$ & $6.48 \pm 0.10$ & $\ldots$ & $\ldots$ & \\
\hline $174 \ldots \ldots \ldots \ldots \ldots$ & 707 & 139 & 201 & 053522.20 & -052425.0 & $\ldots$ & $6.71 \pm 0.11$ & $\ldots$ & $\ldots$ & \\
\hline $175 \ldots \ldots \ldots \ldots \ldots$ & 710 & 155 & 224 & $05 \quad 3522.32$ & -052414.5 & $\ldots$ & $6.66 \pm 0.11$ & $\ldots$ & $\ldots$ & \\
\hline $176 \ldots \ldots \ldots \ldots \ldots \ldots$ & 721 & 233 & 319 & 053522.54 & -052343.8 & $\ldots$ & . & $7.90 \pm 0.3$ & $\ldots$ & \\
\hline 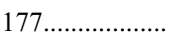 & 723 & 339 & 443 & 053522.80 & -052313.8 & $\ldots$ & $5.82 \pm 0.09$ & $\ldots$ & $\ldots$ & \\
\hline $178 \ldots \ldots \ldots \ldots \ldots \ldots$ & 1993 & 82 & $129 \mathrm{~A}, \mathrm{~B}$ & 053522.90 & -052457.5 & $\ldots$ & $4.97 \pm 0.18$ & $\ldots$ & $\ldots$ & \\
\hline $179 \ldots \ldots \ldots \ldots \ldots$ & 744 & 262 & 347 & 053523.78 & -052334.6 & $\ldots$ & $\ldots$ & $6.28 \pm 0.3$ & $\ldots$ & \\
\hline $180 \ldots \ldots \ldots \ldots \ldots$ & 9317 & 460 & 601 & 053524.41 & -052231.9 & $\ldots$ & $\ldots$ & $6.41 \pm 0.3$ & $\ldots$ & \\
\hline 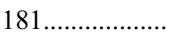 & 756 & 110 & 164 & 053524.44 & -052441.0 & $\ldots$ & $5.50 \pm 0.21$ & $\ldots$ & $\ldots$ & \\
\hline $182 \ldots \ldots \ldots \ldots \ldots \ldots$ & 762 & 119 & 176 & 053524.70 & -052436.0 & $\ldots$ & $6.31 \pm 0.31$ & $\ldots$ & $\ldots$ & \\
\hline $183 \ldots \ldots \ldots \ldots \ldots$ & $766 a, b$ & 387 & 494A, B & 053525.07 & -052259.4 & $\ldots$ & $\ldots$ & $6.01 \pm 0.3$ & $\ldots$ & \\
\hline
\end{tabular}

NoтE.- Units of right ascension are hours, minutes, and seconds, and units of declination are degrees, arcminutes, and arcseconds.

Sources 84 and 87 are unresolved in the $Q$ band.

b From O'Dell \& Wen (1994).

c From Hayward et al. (1994).

${ }^{d}$ The identification number 499 is used the list of Muench et al. (2002) for two different stars (both are binaries). 
correction was applied to each frame. In several cases, the nonuniformity of the background emission dominates the final photometric accuracy. For point sources, an error in the sky estimate affects in a predictable way the results of multiaperture photometry with curve of growth correction: an overestimate of the sky will cause the source to become fainter with the radius and vice versa. We used this property to tune the local sky value to obtain photometric results as much as possible independent of the aperture. Sometimes, however, the variations of the sky brightness underneath the source or the extended pointspread function (PSF) profile produced unstable results. In these cases, we adopted a different strategy. Using the IDL procedure MIN_CURVE_SURF, we estimated the brightness distribution of the sky below the source using the minimum curvature surface fitting a sky annulus surrounding the star. The assumption here is that in the presence of strongly variable background, a higher order interpolation provides a more reliable estimate of the sky distribution under the source. The subtraction of this "sky" frame from the original one provides stellar images that sit on a relatively flat background and therefore can be measured with greater accuracy. Results obtained with this method turned out to depend on the choice of the inner and outer radii of the annulus, typically 5 and 8 pixels, respectively. Our final results were then obtained by averaging the results provided by a few different, but reasonable on the basis of visual inspection, combinations of inner and outer radius. Their scatter has also been added in quadrature to the total uncertainty. In general, photometric measurements obtained with different methods coincide within their errors, especially for the smaller apertures $\left(1^{\prime \prime}-1\right.$."5) and within the same run. The uncertainties we quote combine quadratically the uncertainties of the zero points $(0.12 \mathrm{mag}$ at $7.7 \mu \mathrm{m}, 0.06 \mathrm{mag}$ at $8.7 \mu \mathrm{m}, 0.07 \mathrm{mag}$ at $9.6 \mu \mathrm{m}, 0.06 \mathrm{mag}$ at $10.2 \mu \mathrm{m}, 0.05 \mathrm{mag}$ at $11.6 \mu \mathrm{m}, 0.05 \mathrm{mag}$ at $12.4 \mu \mathrm{m}$, and 0.06 mag in the broad $N$ band, and 0.11 mag in the $Q$ band), the source photon statistics and sky uniformity, and the scatter between the best estimates obtained with different methods or between different choices of radius/annulus, as explained.

All sources that had photometric measurements different between the two runs by an amount comparable to or larger than the quoted $1 \sigma$ error were double-checked. A direct comparison with nearby sources provides in several cases strong evidence for source variability. For sources that are isolated or superposed on a large and complex background (observed with different chopping throws or orientation in the two runs), it is not possible to safely discriminate between variability and unaccounted photometric errors. Whereas the main photometric catalog has been obtained analyzing the single individual images before mosaicking and reconstruction, we have also examined the final reconstructed mosaics, both as a sanity check and to find possible evidence of faint sources that may appear only after all frames have been stacked together.

Concerning the overall sensitivity, the final signal-to-noise ratios of our reconstructed mosaics depend on a number of factors such as the integration time of each exposure, the number of overlaying exposures contributing at each point, air mass, background brightness, chopping direction, and noise propagation in the reconstruction algorithm. Therefore, the sensitivity and completeness limits vary across the field in a complex way. To provide an indicative value of our sensitivity limit, we observe that our catalog of $10 \mu \mathrm{m}$ sources contains objects down to $[N] \simeq 8 \mathrm{mag}$ ( $\operatorname{see} \oint 4.5$ ). Since all stars brighter than $[N] \simeq 6.0$ mag are well detected in all partial mosaics, we can assume $6.0 \mathrm{mag}$ as our completeness limit at $10 \mu \mathrm{m}$, at least in the central $2^{\prime} \times 2^{\prime}$ of our field. However, according to this criterion, this value rises to $[N] \simeq 5$ in the immediate vicinity of the Trapezium, where the nebular emission becomes stronger. This is because six sources in the range $[N]=5.0-6.0$ have been detected only with favorable chopping throw and are measured only at one epoch. For the narrowband images, a recursive standard deviation algorithm with sigma clipping gives, over an empty sky area at the southeast corner of our images, the following values, scaled over $1^{\prime \prime}$ area: $13.1 \mathrm{mJy}$ at $7.7 \mu \mathrm{m}$, $4.3 \mathrm{mJy}$ at $8.7 \mu \mathrm{m}, 11.6 \mathrm{mJy}$ at $9.6 \mu \mathrm{m}, 6.8 \mathrm{mJy}$ at $10.2 \mu \mathrm{m}$, $7.5 \mathrm{mJy}$ at $11.6 \mu \mathrm{m}$, and $9.7 \mathrm{mJy}$ at $12.4 \mu \mathrm{m}$.

\section{RESULTS}

The final reconstructed images are shown in Figure $2(10 \mu \mathrm{m})$ and Figure $3(20 \mu \mathrm{m})$. The size of the final $10 \mu \mathrm{m}$ mosaic is 1144 pixels $\times 1072$ pixels, corresponding to approximately 5. $0 \times$ 4.7. However, since we were able to complete only the central columns to their originally planned extent, the rectangular area fully imaged is smaller, i.e., 1144 pixels $\times 800$ pixels $=5$.' $^{\prime} \times$ 3.5. The $20 \mu \mathrm{m}$ image has a size of 1144 pixels $\times 702$ pixels, corresponding to approximately $5 . ' 0 \times 3$. $^{\prime} 0$.

The morphology at $10 \mu \mathrm{m}$ is clearly dominated by the $\mathrm{BN} / \mathrm{KL}$ complex and by the Ney-Allen Nebula, the two bright sources visible at the center of the field (BN/KL to the north, Ney-Allen to the south). Extended emission permeates the entire region, with a general decrease of surface brightness with increasing angular distance from the center. This intense, diffuse emission is barely visible in the original, nonreconstructed images, since the chopping and nodding process filters low-frequency spatial components. At $20 \mu \mathrm{m}$, the BN/KL complex dominates. Several spatially resolved knots of diffuse emission are also visible at both wavelengths. The "bright bar," a PDR viewed edge-on approximately $2^{\prime}$ to the southwest of the Trapezium, is visible at the bottom left corner of our $10 \mu \mathrm{m}$ image, in the vicinity of $\theta^{2}$ Ori A (source MAX 172). Several other filaments are visible at $10 \mu \mathrm{m}$ in the nebular background. Their lengths range from $\sim 10^{\prime \prime}$ to $\sim 1^{\prime}$, and their surface brightness is comparable, with peaks in the range $\sim 0.1-0.2 \mathrm{Jy} \operatorname{arcsec}^{-2}$ at $10 \mu \mathrm{m}$. In the central region the peak brightness of the clumps and filaments rises to $\sim 0.7 \mathrm{Jy} \mathrm{arcsec}^{-2}$, in particular to the southwest and to the northwest of the Ney-Allen Nebula and to the south of $\mathrm{BN} / \mathrm{KL}$, in the vicinity of the OMC-1 South region (see $\S 4.4)$.

The most remarkable new feature is represented by three, possibly four, large arcs of mid-IR emission located approximately $1^{\prime}$ to the south of the Trapezium/Ney-Allen Nebula, at R.A.(J2000) $=5^{\mathrm{h}} 35^{\mathrm{m}} 15^{\mathrm{s}}$, decl.(J2000) $=-5^{\circ} 24^{\prime} 10^{\prime \prime}$ (Fig. 4). They point toward the Trapezium stars (see also Fig. 12 for a sketch of their location inside the nebula) and appear brighter on the side facing the Trapezium. These structures are also visible at shorter wavelengths, in particular in the Hubble Space Telescope (HST) WFPC2 image of O'Dell \& Wong (1996), and most probably represent surfaces illuminated by grazing UV flux from the central stars.

\subsection{The Trapezium Region}

Our 10 and $20 \mu \mathrm{m}$ images of the Trapezium region (Fig. 5) show, with improved sensitivity and spatial resolution, the general morphology first observed at $10 \mu \mathrm{m}$ by McCaughrean \& Gezari (1991) and studied in more detail by Hayward et al. (1994). The extended mid-IR emission discovered by Ney \& Allen (1969) is resolved into a number of diffuse arclike structures pointing toward $\theta^{1}$ Ori $\mathrm{C}$. The brightest one, corresponding to the brightness peak of the Ney-Allen Nebula, is centered on the $\mathrm{B} 0 \mathrm{~V} \operatorname{star} \theta^{1}$ Ori $\mathrm{D}$, undetected in our images. In the rest 


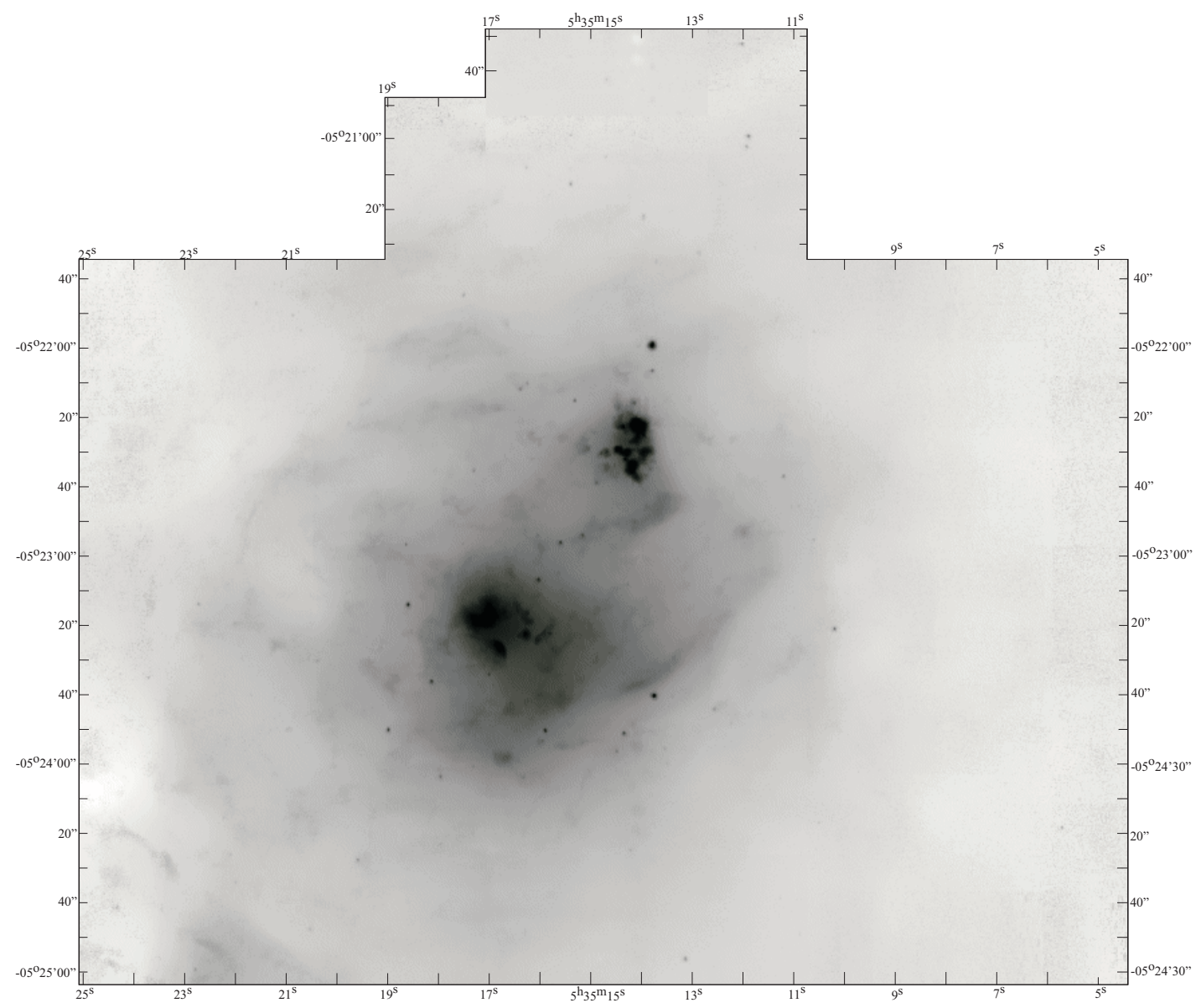

FIG. 2.-Final $10 \mu \mathrm{m}$ mosaic of the Orion Nebula. The two saturated sources at the center of the field are (1) to the southeast, the Ney-Allen Nebula, in correspondence with the Trapezium stars, and (2) to the northwest, the BN/KL complex.

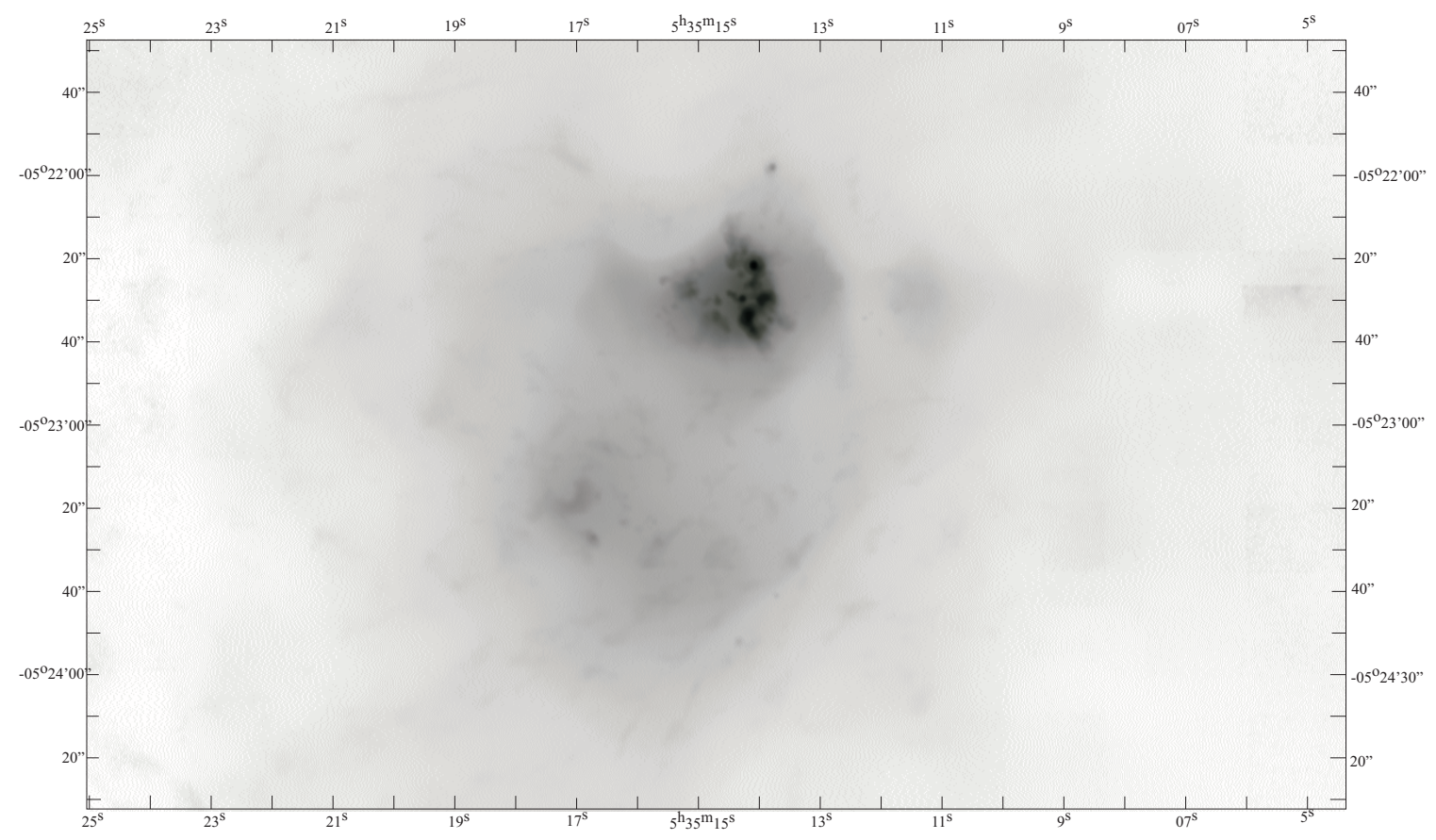

FIg. 3.-Same as Fig. 2, but at $20 \mu \mathrm{m}$. The only prominent source at this wavelength is the BN/KL complex. 

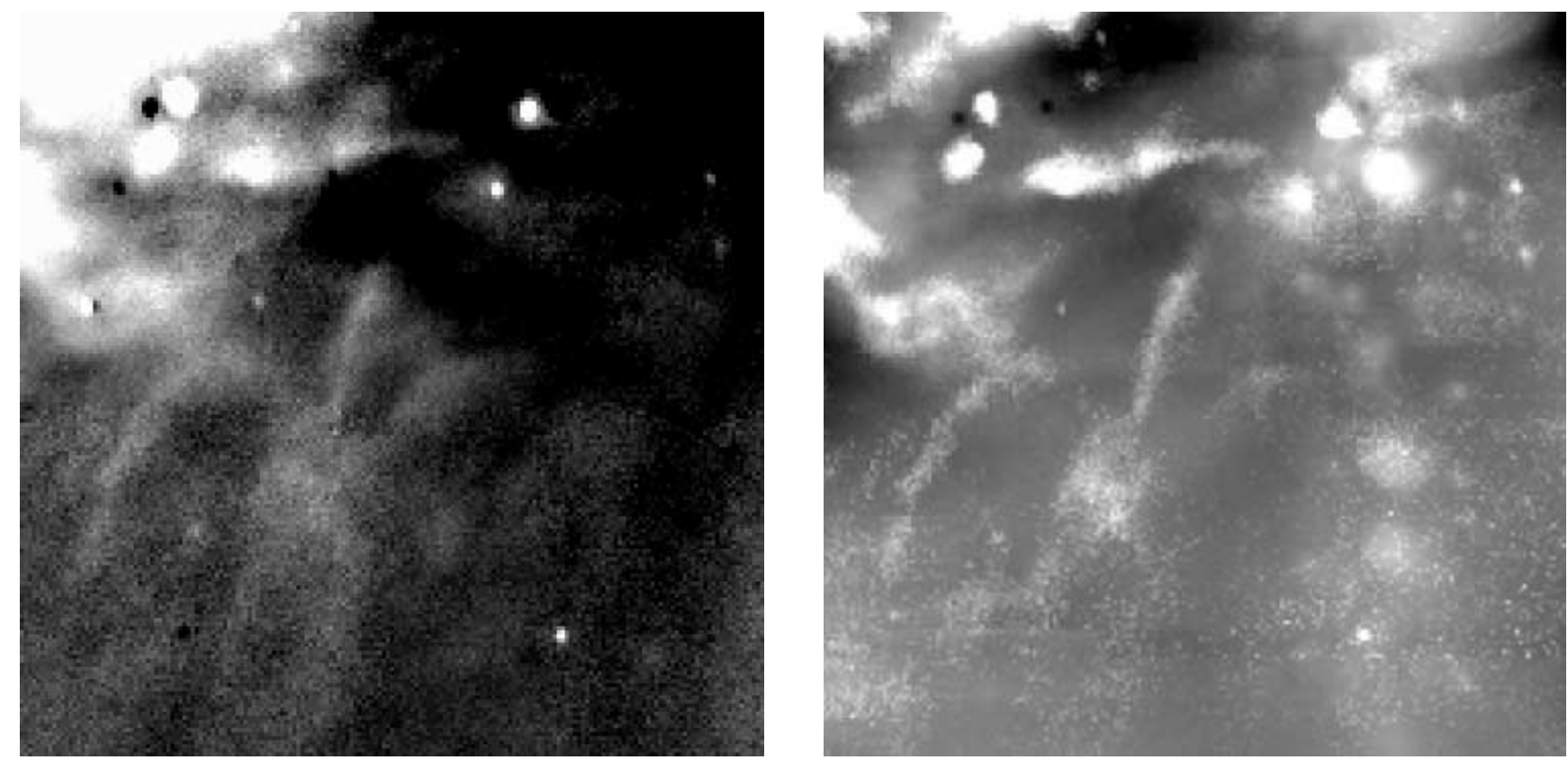

FIG. 4.-Arcs of mid-IR emission approximately $45^{\prime \prime}$ south of the Trapezium (see Fig. 11 for their location). Left: Original image before image reconstruction. Right: Same field after image reconstruction and filtering. Scale is $45^{\prime \prime} \times 45^{\prime \prime}$, with standard orientation. The sources in the northwestern corner fall within the OMC-1 South region (see Fig. 11).

of this paper, we use the name Ney-Allen Nebula to indicate this feature only. Another five arcs encircle the sources LV 1-LV 5 of Laques \& Vidal (1979), the first photoionized disks observed in the vicinity of $\theta^{1}$ Ori $C$.

All arcs are spatially resolved in our images and display different geometries and surface brightnesses. A most remarkable object lies in the immediate vicinity of $\theta^{1}$ Ori $\mathrm{C}$. This is the circular knot at the center of Figure 5 (left) first identified as SC 3 by Hayward et al. (1994). $\theta^{1}$ Ori C itself is also visible at $10 \mu \mathrm{m}$, but not at $20 \mu \mathrm{m}$, as the fainter source $\simeq 1$ ".8 to the east of SC 3. All together, the arcs appear to trace the edge of a cavity centered on $\theta^{1}$ Ori $\mathrm{C}$, filled with diffuse mid-IR emission, and with surface brightness increasing to the northeast. The morphology at $20 \mu \mathrm{m}$ is similar, but we do not detect the peak that Hayward (1994) found $20^{\prime \prime}$ to the southwest of the Ney-Allen Nebula at wavelengths longer than $20 \mu \mathrm{m}$.

\subsection{The BN/KL Complex}

\subsubsection{Morphology}

In Figure 6 we show the 10 and $20 \mu \mathrm{m}$ enlargements of the $\mathrm{BN} / \mathrm{KL}$ complex from the data taken on 2000 December 18 ,
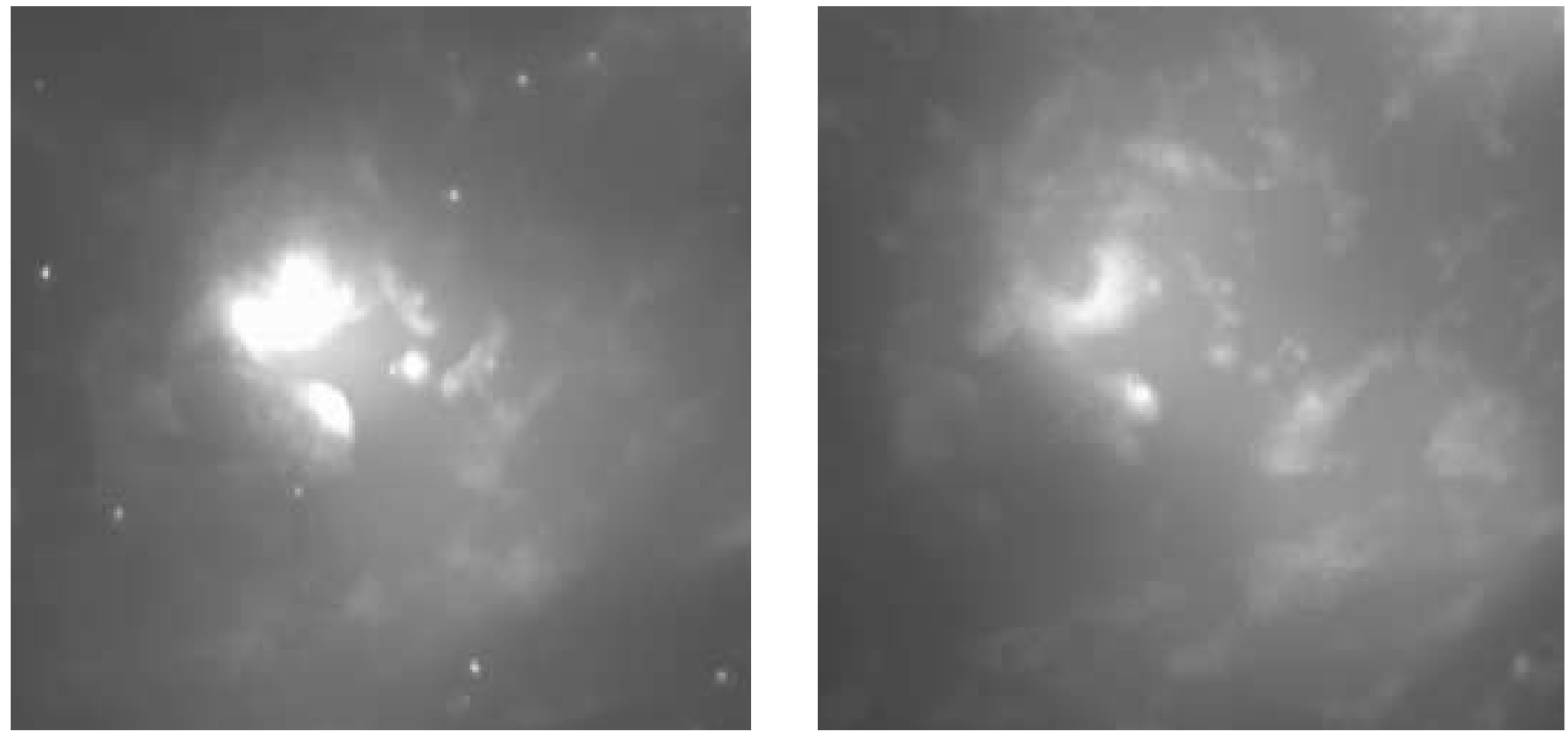

FIG. 5.-Images of the Trapezium region at $10 \mu \mathrm{m}($ left $)$ and at $20 \mu \mathrm{m}$ (right). $\theta^{1}$ Ori C and SC 3 are the two circular sources at the center of the $10 \mu \mathrm{m}$ image, with $\theta^{1}$ Ori $\mathrm{C}$ being the fainter one to the left. Field size is $1^{\prime} \times 1^{\prime}$, and north is up and east is to the left. 

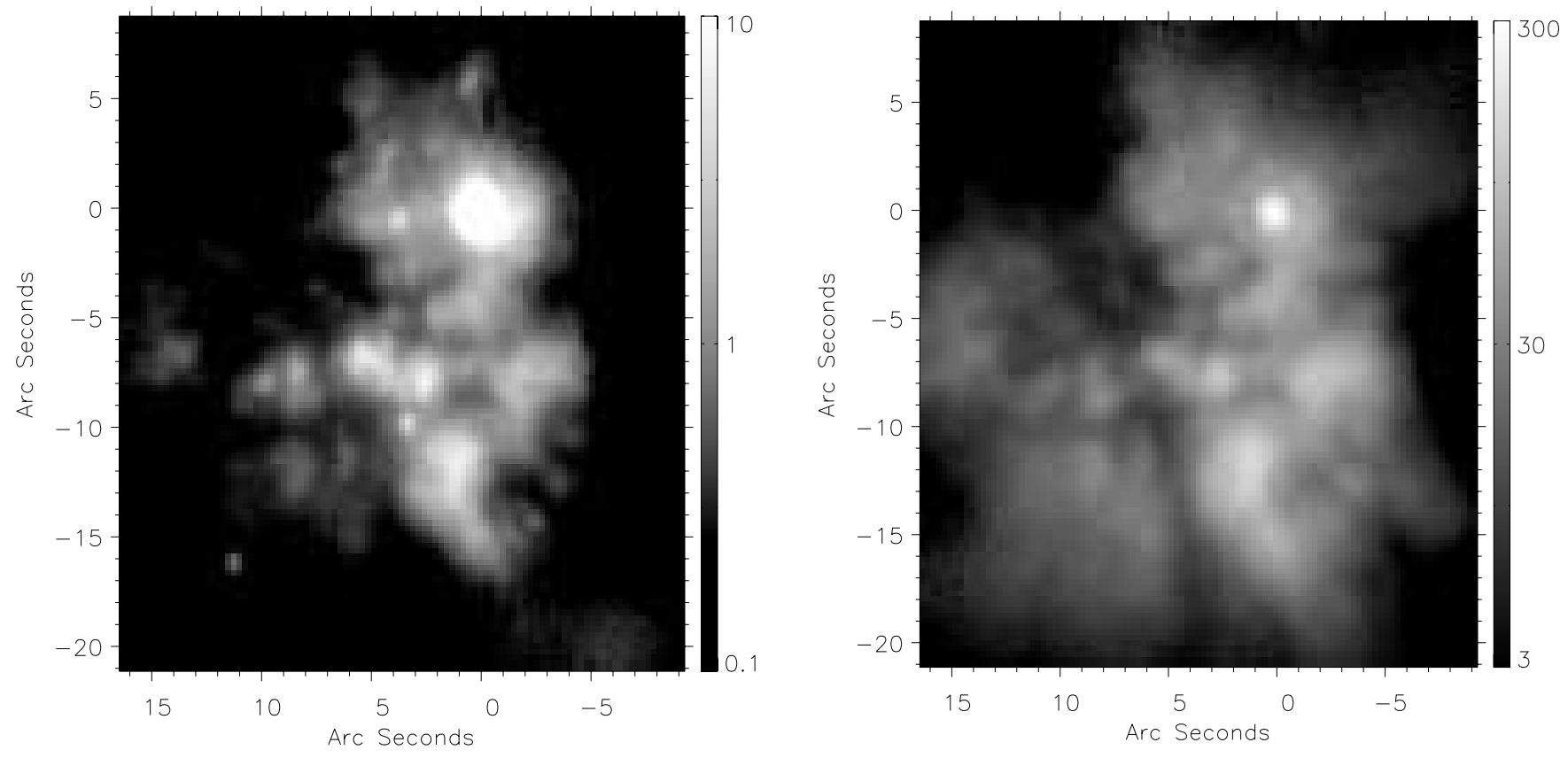

Fig. 6. - Images of the BN/KL complex at $10 \mu \mathrm{m}$ (left) and $20 \mu \mathrm{m}$ (right). The scale is logarithmic and ranges between 0.1 and $10 \mathrm{Jy}$ arcsec ${ }^{-2}$ at $10 \mu \mathrm{m}$ and between 3 and $300 \mathrm{Jy} \operatorname{arcsec}^{-2}$ at $20 \mu \mathrm{m}$.

whereas in Figure 7 the same region is shown in the six narrowband silicate filters. All images are absolutely calibrated and presented in logarithmic scale to enhance the faintest emission across the field.

A few artifacts are present, generally related to the extraordinary brightness of the $\mathrm{BN}$ peak: in the broad $N$-band image (Fig. 6, left there are two ghost images of $\mathrm{BN}$, approximately at $\left(4{ }^{\prime \prime} 5,-0.5\right)$ and $\left(0.5,66^{\prime \prime}\right)$, caused by inner reflections in the camera optics (see also Fig. 8, left). Bandwidth limitations of the readout electronics create in all narrowband images an anomalous "spike," approximately 4 pixels wide, protruding to the west of BN. Some extra noise is also visible in the highly stretched $N$ - and $Q$-band images as a horizontal periodic pattern and in the vertical direction in the rows and columns corresponding to the BN peak. In general, however, the images have excellent quality and are diffraction-limited at all wavelengths $(\lambda / D$ on UKIRT ranges from $0 . " 41$ at $7.7 \mu \mathrm{m}$ to 0.67 at $12.4 \mu \mathrm{m}$ and 1.08 at $19.9 \mu \mathrm{m}$ ). Because of the high surface brightness of the region, it is not easy to see the spectacular diffraction pattern of $\mathrm{BN}$, with the exception of the first ring shown saturated in all images except the $Q$ band. Several diffraction rings appear in the differential images that we will present in $\S 4.3$. To preserve the highest resolution, considering also that we are mostly dealing with the surface brightness of extended objects, we have not rebinned or convolved our images to our lowest resolution. In Figure 9 we show a color composite image of the BN/KL complex obtained combining our $7.7 \mu \mathrm{m}$ (blue), $12.4 \mu \mathrm{m}$ (green), and $19.9 \mu \mathrm{m}$ (red) images.

Early mid-IR studies (Becklin \& Neugebauer 1967; Rieke et al. 1973) resolved this region into eight sources (IRc1-IRc8; $\mathrm{BN}$ corresponding to IRc1). Another 10 compact sources were added by Gezari et al. (1998) to the IRc list, and recently Shuping et al. (2004) added five more. Our images have resolution and sensitivity comparable to those of Greenhill et al. (2004) and Shuping et al. (2004) but cover the entire field in eight bands. Our narrowband $12.4 \mu \mathrm{m}$ image (Fig. 7, bottom right), in particular, is comparable to Figure 1 of Shuping et al. (2004) and Figure 2 of Greenhill et al. (2004). We detect new features, especially in the unexplored outer regions of the complex. Like the large majority of compact sources already cataloged, all new features appear extended and resolved into clumps, ridges, and arclike structures down to our $\sim 0$ ". 5 resolution limit. Instead of adding new entries to the already long list of IRc sources, we indicate the new sources using their offset ( $\Delta$ R.A.", $\Delta$ decl." $)$ relative to BN. For the $30 \mathrm{IRc}$ sources and subcomponents already classified, we still use the list of Shuping et al. (2004). The position of the most relevant sources is indicated in Figure 8.

$\mathrm{BN}$, the main luminosity peak longward of $2 \mu \mathrm{m}$, remains unresolved at all wavelengths. Gaussian fits to the image profile at $10 \mu \mathrm{m}$ show a $\simeq 3.2$ pixel FWHM both in right ascension and declination, corresponding to $\mathrm{FWHM} \simeq 0$ "' 87 . For an IR source with a spectrum increasing with wavelength, like $\mathrm{BN}$, the diffraction limit in broadband filters is approximately set by the longest wavelength passing through the filter, $\lambda_{\max } \simeq$ $13 \mu \mathrm{m}$ in our case. For an effective telescope diameter $D=$ $3.7 \mathrm{~m}$, it is $\lambda / D=00^{\prime \prime} 72$. The pixel sampling and the mosaicking process, performed by integer pixels, add another $\simeq \frac{1}{2}$ pixel of image blur, bringing the FWHM right to the measured value. The BN peak sits on top of an extended clumpy plateau. Shuping et al. (2004) have already identified the remarkable southwest arc, and we just add to the list a compact source immediately to the south at $\left(-2^{\prime \prime}, 2^{\prime \prime}\right)$.

Source $n$ of Lonsdale et al. (1982), coincident with a bipolar radio source resolved by Menten \& Reid (1995; their source "L"), has been recently analyzed in detail by Greenhill et al. (2004) and Shuping et al. (2004). Using LWS on Keck, both teams find that source $n$ is extended, with a FWHM of approximately $\left(00^{\prime \prime} 75 \times 0\right.$ " 50$) \pm 0$ "' 10 and major axis oriented at $\simeq 120^{\circ}$. Our pixel scale, approximately 3 times larger than that of LWS, does not sample adequately the core of the diffraction figure at $7.7 \mu \mathrm{m}$. A two-dimensional Gaussian fit at this wavelength gives for source $n$ a FWHM of $\left(00^{\prime \prime} 47 \times 0.51\right)$, but it 

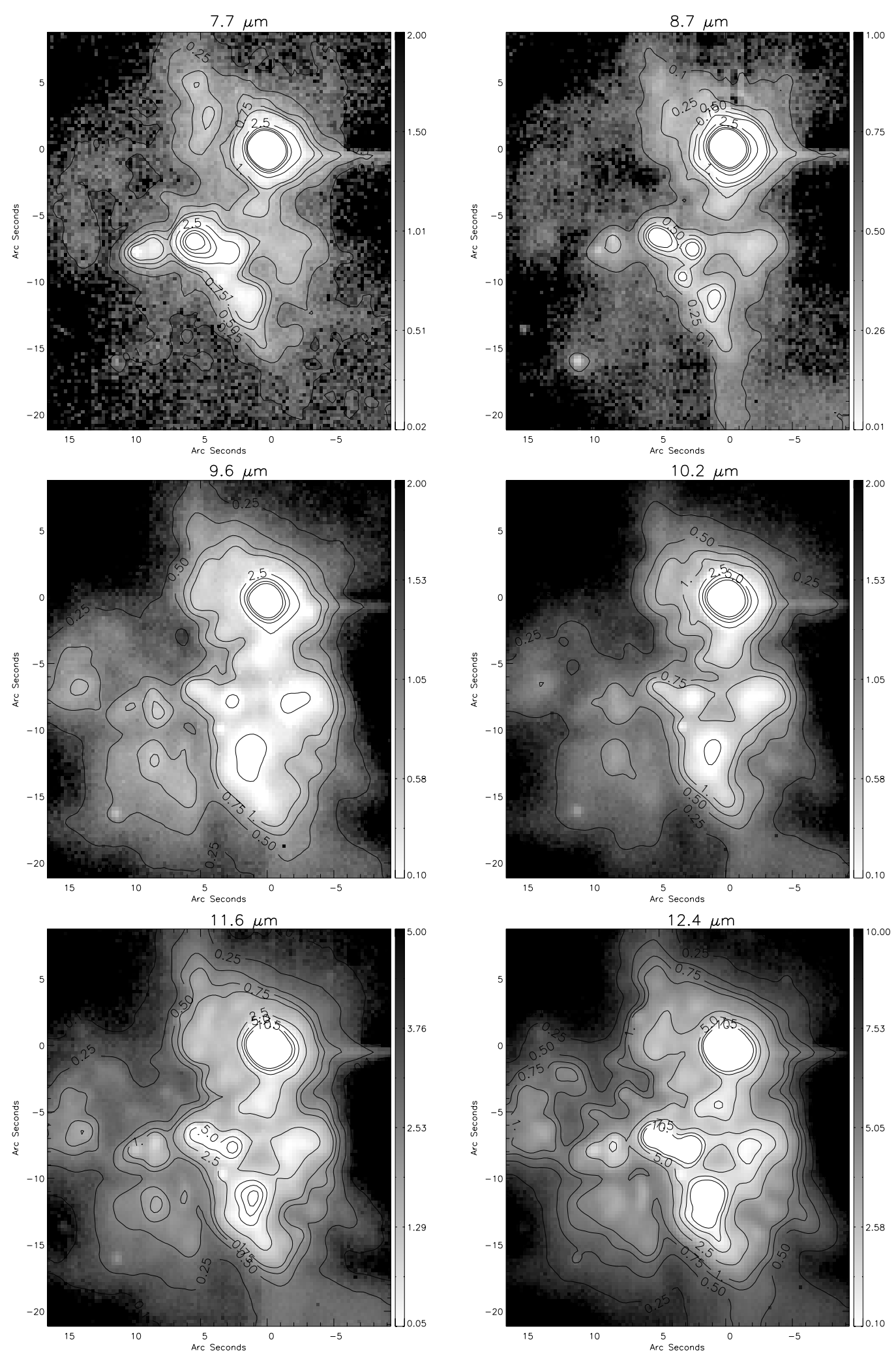

FIG. 7.- Images of the BN/KL complex at 7.7 and $8.7 \mu \mathrm{m}$ (top), 9.6 and $10.2 \mu \mathrm{m}$ (middle), and 11.6 and $12.4 \mu \mathrm{m}$ (bottom). All images are shown in logarithmic scale, and units are janskys per square arcsecond. To facilitate the comparison, all contours are plotted at the same levels of $0.1,0.25,0.50,0.75,1,2.5,5,7.5$, and $10 \mathrm{Jy} \operatorname{arcsec}^{-2}$. Equatorial coordinates are relative to the peak of the BN source (see $\S 2.2 .2$ ).

must be remarked that the Airy function is poorly reproduced by a Gaussian curve. If we use the BN peak as a reference for the PSF core and compare horizontal and vertical cuts across both sources, then source $n$ remains unresolved at all wavelengths in our images.
Two other sources, IRc12 and IRc7, appear pointlike at $7.7 \mu \mathrm{m}$ but become extended at longer wavelengths. Sources IRc11 and IRc6, on the other hand, show compact cores at $12.4 \mu \mathrm{m}$. The point source MAX 69 (see $\S 3.4$ ) is clearly visible at $\left(11^{\prime \prime},-6.5\right)$, $\simeq 20^{\prime \prime}$ southeast of BN. This is a well-known double star which 

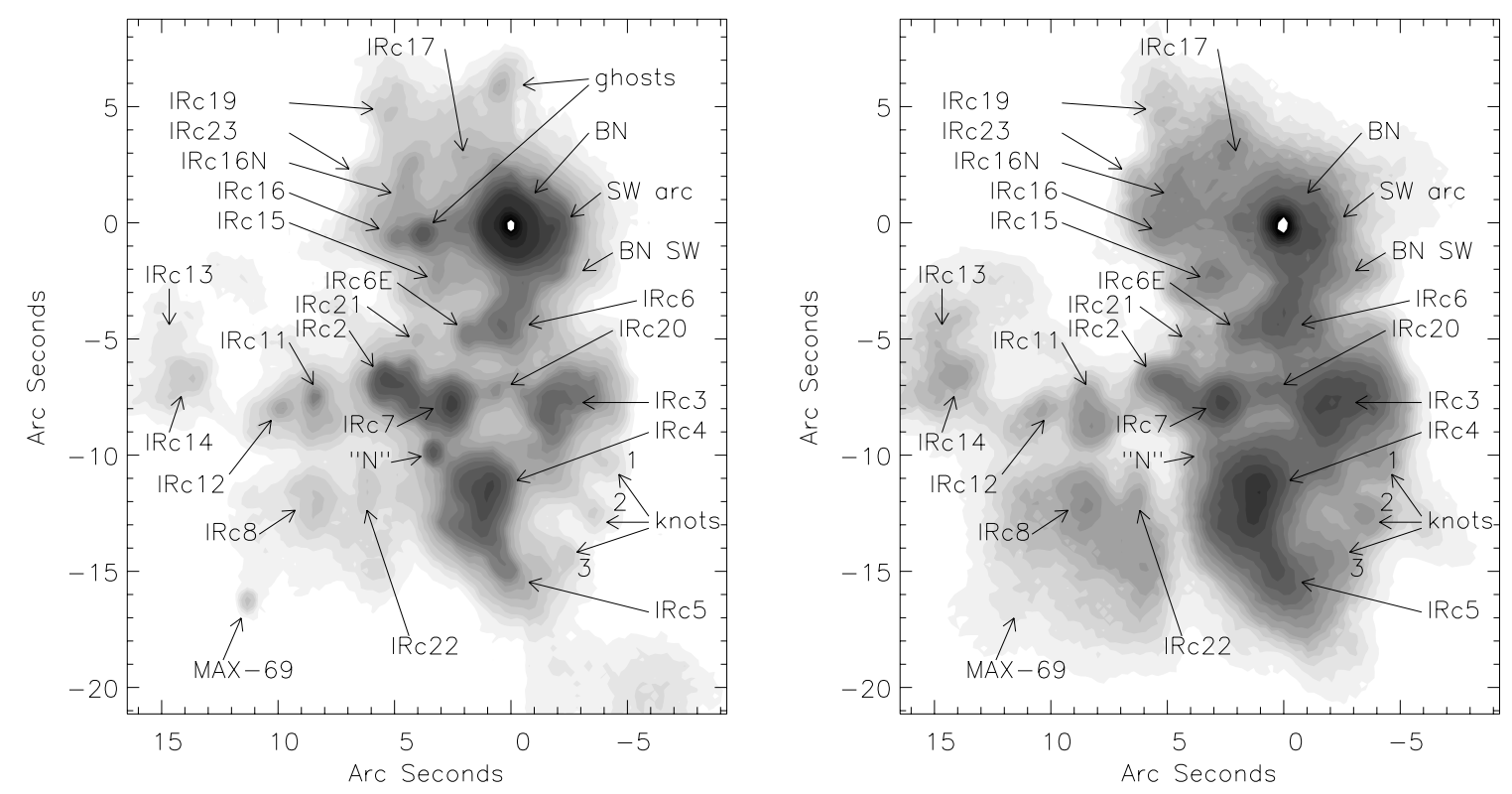

FIG. 8.-Contour plot of the BN/KL complex at $10 \mu \mathrm{m}$ (left $)$ and $20 \mu \mathrm{m}$ (right), with labels indicating the main features discussed in the text. Contour levels are $0.22,0.30,0.37,0.45,0.60,0.80,1.0,1.2,1.35,1.5,1.87,2.25,3.0,4.5,6.0,10,15,30,60,100,150$, and $200 \mathrm{Jy}^{-2} \operatorname{arsec}^{-2}$ at $10 \mu \mathrm{m}$ and $7,10,12,14,16,18,20,25$, $30,35,40,50,60,80,100,120,140,160,180$, and $200 \mathrm{Jy} \mathrm{arcsec}^{-2}$ at $20 \mu \mathrm{m}$.

remains unresolved in our images and most probably lies in the foreground of the $\mathrm{BN} / \mathrm{KL}$ complex.

Diffuse emission becomes brighter to the southeast with longer wavelengths. At $7.7 \mu \mathrm{m}$, sources IRc2, IRc4, and IRc5 appear aligned in the northeast-southwest direction and abruptly truncated to the southeast, but at longer wavelengths this sharp edge, especially for IRc4, becomes less prominent and a broad plateau of emission rises around IRc8 and IRc22. At $19.9 \mu \mathrm{m}$, extended emission fills the entire field and two dark lanes become clearly visible, one oriented north-south along a line roughly passing through positions $\left(8^{\prime \prime}, 0^{\prime \prime}\right)$ and $\left(3^{\prime \prime},-18^{\prime \prime}\right)$, and the other, almost perpendicular, immediately to the south of the "equato-

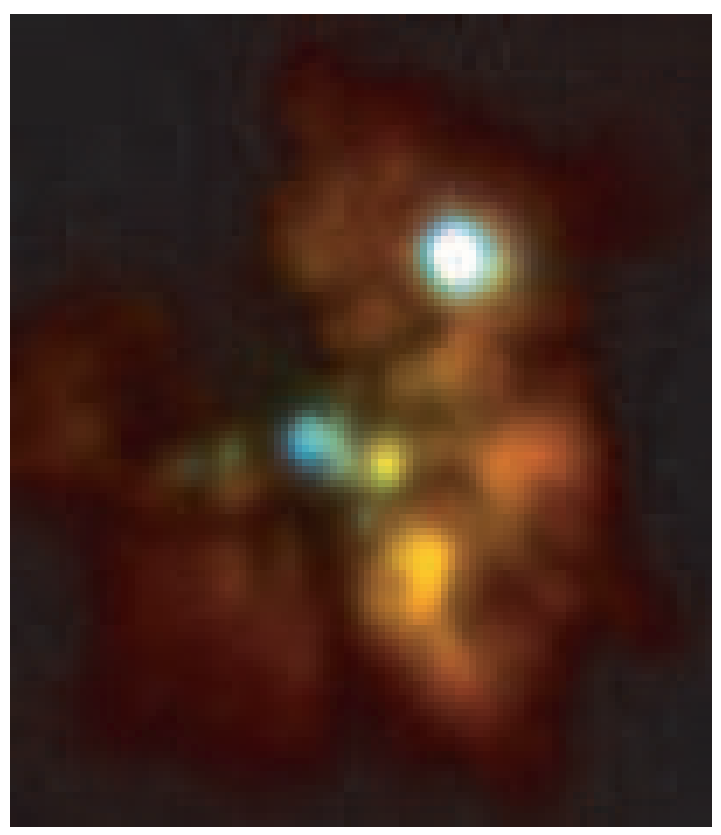

FIG. 9.- Color composite image of the $\mathrm{BN} / \mathrm{KL}$ region obtained combining the $7.7 \mu \mathrm{m}$ (blue), $12.4 \mu \mathrm{m}$ (green), and $19.9 \mu \mathrm{m}$ (red) images. rial belt" composed of IRc14, 12, 11, 2, and 7. These dark lanes are coincident with the ammonia emission of Wilson et al. (2000), which traces the density distribution of the Orion hot core (see Greenhill et al. 2004). Another remarkable feature is the peculiar "eastern ring" of sources centered at $\left(10^{\prime \prime},-4\right.$ " $)$ and traced by IRc16, 15, 6E, 21, 2, 11, 12, 14, and 13, plus other faint emission around $\left(12^{\prime \prime},-2^{\prime \prime}\right)$. The area inside the ring is mostly faint clumpy emission and contains the majority of the $\mathrm{H}_{2} \mathrm{O}$ masers detected by Johnston et al. (1989) in OMC-1. The similar shape of the prominent IRc3 and IRc4 sources is also noticeable; both are arclike and point to the IRc2 region. The tails of their arcs are part of another remarkable new feature, a group of equally spaced knots tracing an arclike "necklace" between IRc5 and IRc3. The knots have all comparable brightness, are elongated and radially oriented, and point to the IRc2 central region.

Finally, IRc2 is well resolved at $10 \mu \mathrm{m}$ (Fig. 6, left) and shows a morphology that matches the structure of sources IRc2-A to IRc2-D resolved by Chelli et al. (1984) and Dougados et al. (1993) at shorter wavelengths. Its morphology has been discussed in detail by Greenhill et al. (2004) and Shuping et al. (2004). We will return to this object in $\S 4.3$.

\subsubsection{Photometry}

In Table 4, we present the photometric data at 35 positions within the $\mathrm{BN} / \mathrm{KL}$ complex. The first 30 are the sources listed in Table 1 of Greenhill et al. (2004), and the last five are the three brightest knots to the southwest of IRc4 (knots 1-3), the compact source south of the southwest arc (BN SW), and the star MAX 69. Photometry has been obtained using a circular aperture of 1 ". 128 diameter, corresponding to $2 \operatorname{arcsec}^{2}$ area, but the fluxes are normalized to janskys per square arcsecond. The only exception is the $\mathrm{BN}$ peak, for which we give the total flux within a synthetic aperture of 5"2. All values are uncorrected for the background emission, and the zero-point errors listed in $\S 2.2 .3$ represent the main source of uncertainty. The corresponding spectra are shown in Figure 10. The silicate absorption feature is prominent at all positions except for 
TABLE 4

Peak Surface Brightness of the BN/KL Complex

\begin{tabular}{|c|c|c|c|c|c|c|c|c|c|c|}
\hline \multirow[b]{2}{*}{ Source Name } & \multirow[b]{2}{*}{ OFFset R.A. } & \multirow[b]{2}{*}{ Offset Decl. } & \multicolumn{6}{|c|}{ NARROWBAND } & \multicolumn{2}{|c|}{ BROADBAND } \\
\hline & & & $7.7 \mu \mathrm{m}$ & $8.7 \mu \mathrm{m}$ & $9.8 \mu \mathrm{m}$ & $10.2 \mu \mathrm{m}$ & $11.2 \mu \mathrm{m}$ & $12.4 \mu \mathrm{m}$ & $N$ Band & $Q$ Band \\
\hline$\ldots \ldots \ldots \ldots . . .$. & 0.0 & 0.0 & 122.30 & 106.11 & 62.59 & 57.21 & 126.80 & 143.19 & 119.58 & 171.34 \\
\hline 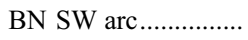 & -1.4 & -0.6 & 9.82 & 11.35 & 7.22 & 10.31 & 16.29 & 20.78 & 8.65 & 59.76 \\
\hline IR n & 3.3 & -9.8 & 2.11 & 1.06 & 1.62 & 1.22 & 3.23 & 7.14 & 2.12 & 31.53 \\
\hline 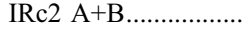 & 5.7 & -7.0 & 15.21 & 1.07 & 1.38 & 0.89 & 5.62 & 20.24 & 3.52 & 34.07 \\
\hline IRc2 C & 4.6 & -7.8 & 7.98 & 0.73 & 1.50 & 0.95 & 4.67 & 14.94 & 2.77 & 36.67 \\
\hline 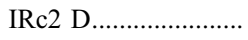 & 4.8 & -7.0 & 10.37 & 1.58 & 1.59 & 1.14 & 6.67 & 19.64 & 3.85 & 41.70 \\
\hline 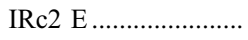 & 4.5 & -6.3 & 4.55 & 1.08 & 1.02 & 0.86 & 3.96 & 10.17 & 1.86 & 28.45 \\
\hline IRc3 N............................. & -1.6 & -7.2 & 0.62 & 0.44 & 2.32 & 1.64 & 4.02 & 7.75 & 1.94 & 66.69 \\
\hline 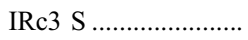 & -1.4 & -7.9 & 0.69 & 0.43 & 2.71 & 1.75 & 4.32 & 8.81 & 2.23 & 74.34 \\
\hline 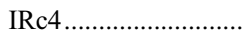 & 1.1 & -11.1 & 1.63 & 0.83 & 3.58 & 2.72 & 10.13 & 20.15 & 4.76 & 101.36 \\
\hline IRc5 & 0.5 & -14.5 & 0.56 & 0.30 & 2.08 & 1.30 & 3.45 & 7.56 & 1.73 & 59.99 \\
\hline IRc6 & 0.4 & -4.5 & 0.91 & 0.42 & 2.13 & 1.40 & 3.92 & 7.74 & 2.11 & 54.24 \\
\hline 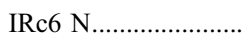 & -0.2 & -3.2 & 0.89 & 0.61 & 2.30 & 1.64 & 3.58 & 5.97 & 1.99 & 52.92 \\
\hline 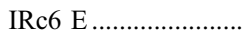 & 1.8 & -4.8 & 0.75 & 0.24 & 1.43 & 0.96 & 2.81 & 6.13 & 1.52 & 38.26 \\
\hline IRc7 ......................... & 2.8 & -7.8 & 4.29 & 1.21 & 2.84 & 1.89 & 8.86 & 23.88 & 4.93 & 73.93 \\
\hline IRc8 & 8.4 & -12.2 & 0.13 & 0.07 & 1.02 & 0.63 & 1.07 & 2.04 & 0.58 & 28.24 \\
\hline IRc11 ....................... & 8.5 & -7.4 & 1.38 & 0.32 & 0.89 & 0.64 & 1.90 & 5.28 & 1.19 & 20.76 \\
\hline IRc12 ............................ & 9.9 & -7.9 & 1.58 & 0.22 & 0.72 & 0.51 & 1.45 & 3.64 & 0.85 & 16.94 \\
\hline IRc13 & 14.1 & -4.8 & 0.14 & 0.06 & 0.56 & 0.35 & 0.55 & 1.14 & 0.29 & 14.37 \\
\hline 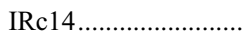 & 13.8 & -6.7 & 0.18 & 0.09 & 0.78 & 0.49 & 0.96 & 1.87 & 0.52 & 19.42 \\
\hline IRc15 ........................... & 3.1 & -2.3 & 0.45 & 0.23 & 1.30 & 0.87 & 1.91 & 3.46 & 1.15 & 33.98 \\
\hline 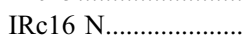 & 4.9 & 1.3 & 0.71 & 0.24 & 1.17 & 0.81 & 1.83 & 3.09 & 0.99 & 30.66 \\
\hline 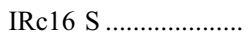 & 5.0 & -0.3 & 0.51 & 0.18 & 1.17 & 0.77 & 1.77 & 3.26 & 1.37 & 31.12 \\
\hline 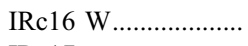 & 4.0 & 0.2 & 0.47 & 0.23 & 1.20 & 0.83 & 1.61 & 2.73 & 1.58 & 30.73 \\
\hline 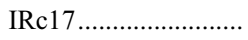 & 3.0 & 2.3 & 0.42 & 0.26 & 1.17 & 0.77 & 1.35 & 2.08 & 0.69 & 28.38 \\
\hline 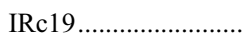 & 5.2 & 4.7 & 0.74 & 0.24 & 0.68 & 0.44 & 0.97 & 1.37 & 0.56 & 16.06 \\
\hline IRc20 & 0.7 & -6.9 & 0.74 & 0.31 & 1.36 & 0.90 & 2.22 & 5.17 & 1.10 & 37.28 \\
\hline 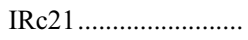 & 4.4 & -5.0 & 0.84 & 0.19 & 0.64 & 0.46 & 1.29 & 3.21 & 0.67 & 16.82 \\
\hline 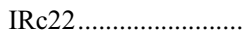 & 6.4 & -11.3 & 0.23 & 0.08 & 0.72 & 0.46 & 0.76 & 1.83 & 0.50 & 17.30 \\
\hline IRc23 & 6.3 & 2.2 & 0.38 & 0.12 & 0.56 & 0.35 & 0.84 & 1.59 & 0.45 & 15.81 \\
\hline Knot 1 ........................ & -4.2 & -10.4 & 0.17 & 0.07 & 0.96 & 0.53 & 0.82 & 1.68 & 0.42 & 25.13 \\
\hline Knot $2 \ldots \ldots \ldots \ldots \ldots \ldots$ & -3.9 & -12.7 & 0.21 & 0.08 & 0.82 & 0.48 & 0.66 & 1.53 & 0.38 & 23.44 \\
\hline Knot $3 \ldots \ldots \ldots \ldots$ & -2.6 & -14.6 & 0.25 & 0.10 & 0.95 & 0.54 & 0.81 & 1.80 & 0.45 & 25.70 \\
\hline 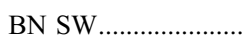 & -2.0 & -2.0 & 1.21 & 0.88 & 1.90 & 1.54 & 3.12 & 5.05 & 1.90 & 36.66 \\
\hline MAX $69 \ldots \ldots \ldots \ldots$ & 11.4 & -16.6 & 0.13 & 0.15 & 0.65 & 0.42 & 0.40 & 0.48 & 0.38 & 11.17 \\
\hline
\end{tabular}

the very last object, MAX 69, which is probably a foreground member of the Trapezium cluster. A secondary maximum at the center of the absorption feature is also generally present, with the exception of the main $\mathrm{BN}$ peak and its immediate surroundings. This emission feature sits at the center of the telluric ozone absorption band and, especially at the lowest signal levels, may be hard to detect in less than excellent weather conditions. Having carefully checked our absolute calibration and verified that our photometry for the main $\mathrm{BN}$ peak agrees with the previously published spectrophotometric data (see $\S 4.3$ ), we believe that this emission feature is real.

\subsection{OMC-1 South}

The OMC-1 South region, located $\simeq 100^{\prime \prime}$ south of the BN/ KL complex, is characterized by intense outflow activity probably generated by deeply embedded sources (O’Dell 2001a). In Figure 11, we show the position of the point sources found at shorter wavelengths together with the contours of the highvelocity outflow of Rodríguez-Franco et al. (1999b). Part of the field is also visible in Figure 4. The Lada et al. (2000) list of candidate protostellar sources contains five objects in this area, TPSC 1, 2, 16, 48, and 78. Three of them, namely, TPSC 1, 2, and 78, are the sources with the reddest $K-L$ color of the entire $L$-band survey: the first two, undetected in the $K$ band by Lada et al. (2000), have lower limits at $[K-L]>6.09$ mag and $[K-L]>4.90 \mathrm{mag}$, respectively, whereas TPSC 78 has $[K-L]=4.85 \mathrm{mag}$. TPSC 1 is nearly coincident with our source, MAX 61, that shows significant variability at $10 \mu \mathrm{m}$, with $[N]=4.40 \mathrm{mag}$ in 1998 November and $[N]=3.68 \mathrm{mag}$ in 2000 December (see $\S 4.5 .1$ ). Object TPSC 2 is MAX 64, and with $[N] \simeq 5.3 \mathrm{mag}$ it also shows a conspicuous $L-N \simeq$ 3.8 mag color index.

\subsection{Point Sources}

In Figure 12, we map the location of the 177 point sources detected at 10 and $20 \mu \mathrm{m}$. In Table 3, we present their crossidentification, location, and $10 \mu \mathrm{m}$ photometry. In particular, we list their sequential running number (col. [1]) and their entry number in the catalogs of Hillenbrand (1997), Hillenbrand \& Carpenter (2000), and Muench et al. (2002) (cols. [2]-[4]), all at shorter wavelengths; coordinates (cols. [5] and [6]) are derived from our final mosaic with typical accuracy $\simeq 0$ ". 5 , accurate enough to unambiguously identify each source. In a few cases, residual pointing errors at the edges of the mosaic cause larger discrepancies, listed in the last column (col. [11]). 

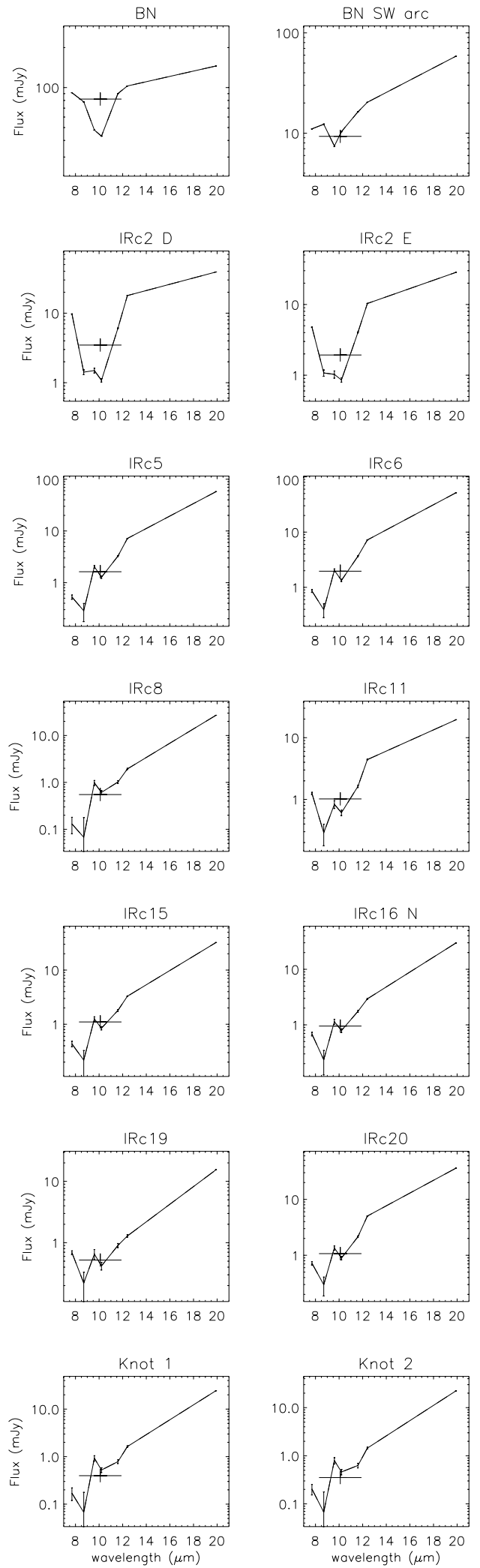
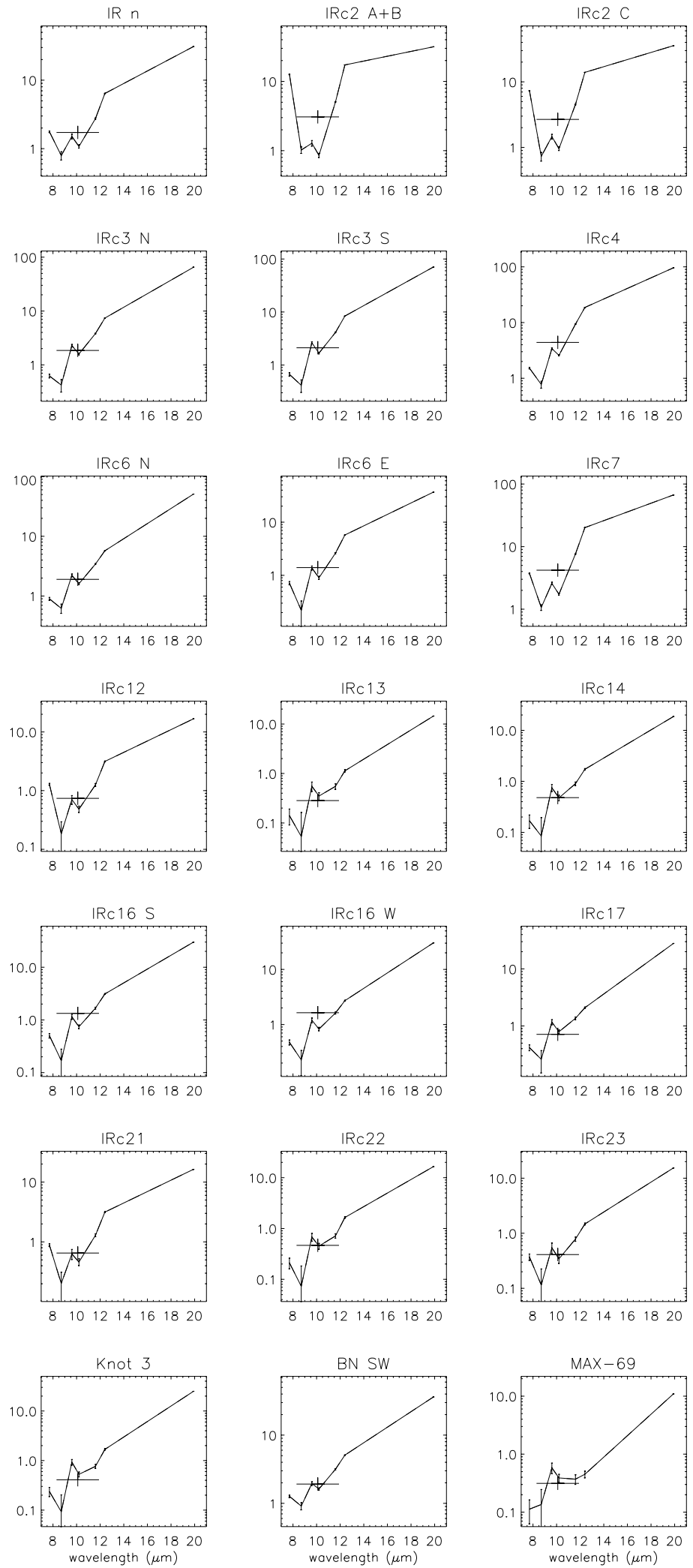

FIg. 10.-Mid-IR spectral energy distribution for the 35 sources of Table 4. Errors refer to the typical zero-point error of our absolute calibration. The horizontal bar indicates the broadband $N$ filter.

The magnitudes are given for the two epochs (cols. [7] and [8]). For a few sources, we could derive photometry only in the final mosaic (column [9]). For 22 sources, there is no counterpart identified at shorter wavelengths. Those without counterparts can therefore be regarded as deeply embedded objects.
A few of them, however, are close to our sensitivity limits and appear only on the final mosaic. As explained in $\S 2.2 .1$, we cannot rule out in these cases the possibility that they are artifacts of our data reconstruction algorithm. Further observations are needed to confirm them as real sources. 


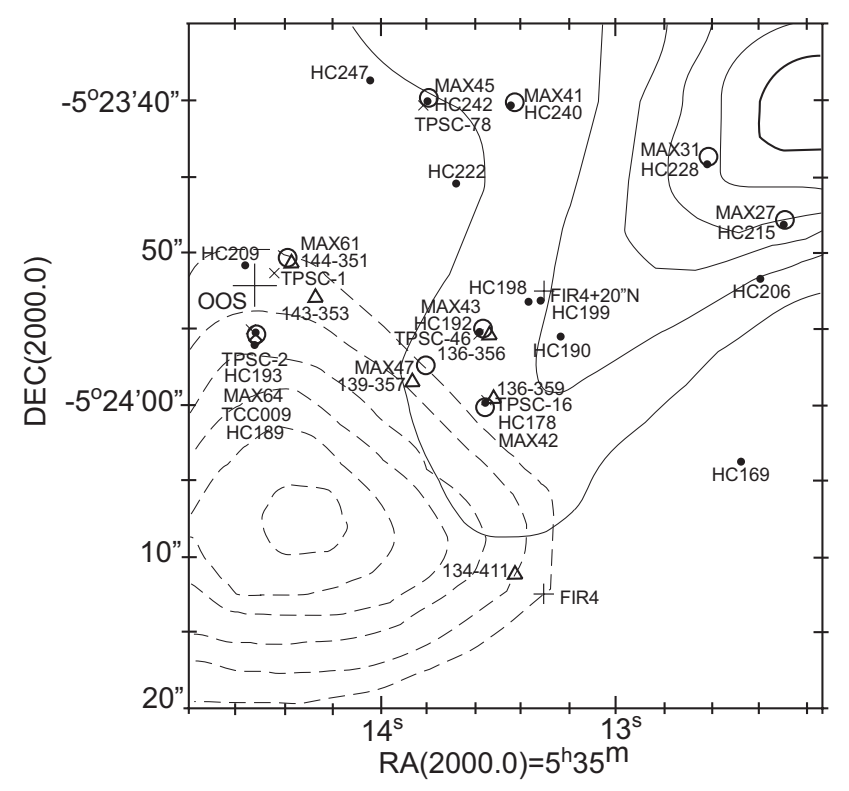

FIG. 11.-Map of the sources located in the OMC-1 South region, together with the intensity contours of the $\mathrm{CO}$ outflow mapped by Rodríguez-Franco et al. (1999b). The naming conventions are: (1) MAX sources: this paper, (2) TPSC sources: Lada et al. (2000), (3) HC sources: Hillenbrand \& Carpenter (2000), (4) 134-411 (et similia) and OOS sources: Zapata et al. (2004), and (5) FIR 4: Rodríguez-Franco et al. (1999a).

\section{DISCUSSION}

\subsection{Diffuse Emission}

With the exception of the bright $\mathrm{BN} / \mathrm{KL}$ complex and the Ney-Allen Nebula, the morphology of the Orion Nebula in the mid-IR is more similar to the radio continuum and dereddened line images obtained by O'Dell \& Yusef-Zadeh (2000) than the optical or near-IR images.

This is because the foreground "veil" of neutral material immediately in front of the nebula, which affects the morphology of the nebula in the visible, becomes optically thin at mid-IR (and radio) wavelengths, whereas the stellar emission, prominent at shorter wavelengths, becomes fainter. For this reason, the "dark bay," a region of high optical depth $\left(A_{V} \geq 3\right)$, approximately parabolic in shape, that is protruding from the east of the nebula up to $\approx 30^{\prime \prime}$ east of the Trapezium, is essentially invisible in our images, confirming its nature as an enhancement of dust column density within the foreground neutral lid (O’Dell \& Yusef-Zadeh 2000).

The filamentary structures seen in emission at $10 \mu \mathrm{m}$ appear to be associated with the extinction-corrected color images of O'Dell \& Yusef-Zadeh (2000). In particular, the "east bright bar" and "E-W bright bar" shown in Figure 5 of O'Dell \& Yusef-Zadeh (2000) have $10 \mu \mathrm{m}$ counterparts. On the other hand, their "north bright bar," which is close to the top edge of our $10 \mu \mathrm{m}$ field, remains undetected, as well as the comet-like

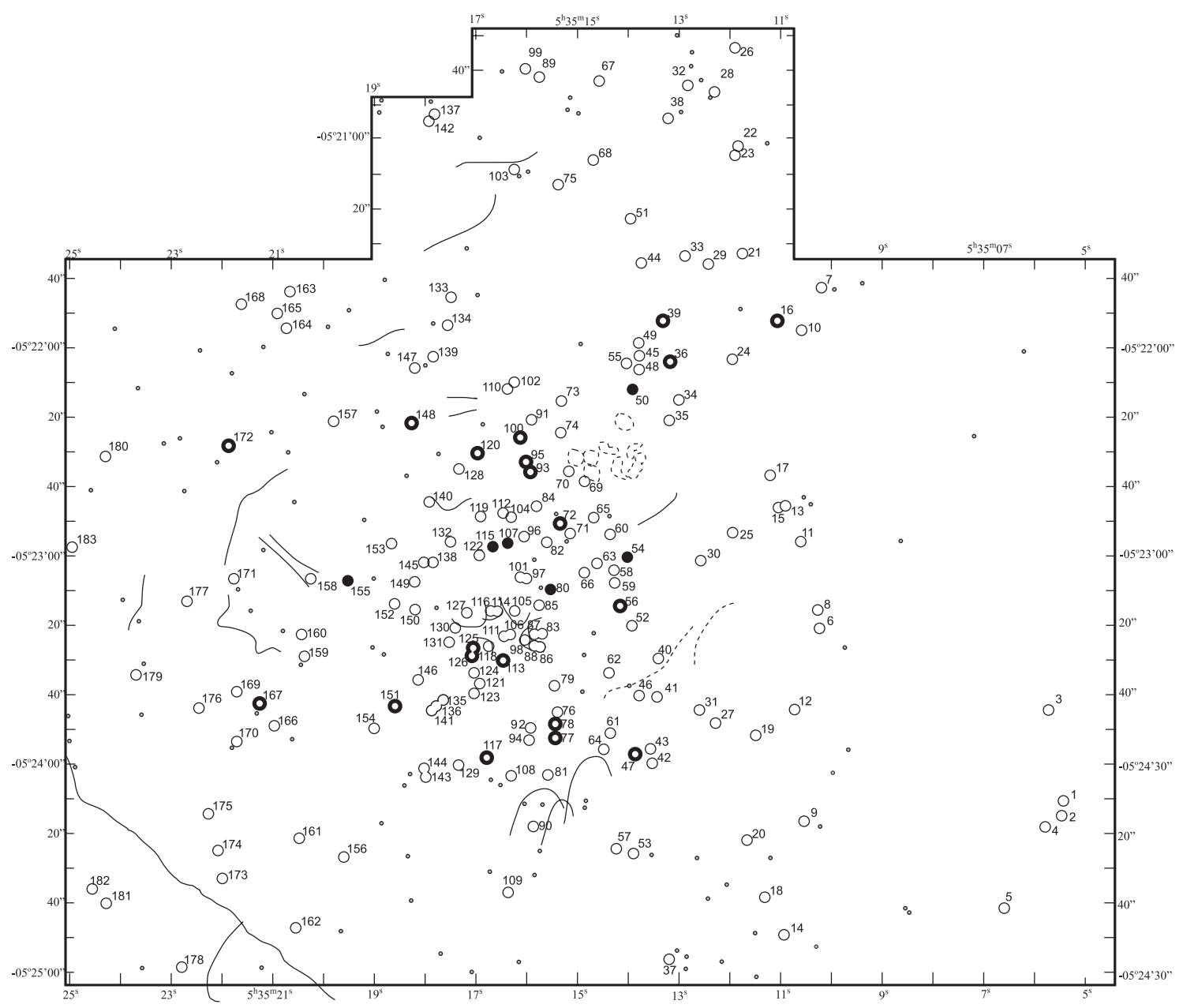

FIG. 12.-Location of our mid-IR point sources. Thin open circles: $10 \mu \mathrm{m}$ sources with counterparts at shorter wavelengths. Thick open circles: $10 \mu \mathrm{m}$ sources detected at $10 \mu \mathrm{m}$ and not at shorter wavelengths. Filled circles: Sources detected only at $20 \mu \mathrm{m}$. Small circles: A subset of the near-IR sources that remained undetected at $10 \mu \mathrm{m}$, presented here for reference and for ease of comparison with near-IR images. Thin solid lines trace the main filamentary structures and the arcs discussed in $\S 4$. $\theta^{1}$ Ori $\mathrm{C}$ is source $111 ; \theta^{2}$ Ori A is source 178. 
structure approximately $1^{\prime}$ to the east of the Trapezium, but they are clearly visible on the $L$-band images of both Lada et al. (2000) and McCaughrean et al. (1996). Most of the $10 \mu \mathrm{m}$ filaments appear aligned along arcs roughly centered on the Trapezium. This result suggests that the filaments trace the edges of the main $\mathrm{H}$ II cavity rather than, e.g., shocks produced by collimated outflows. The morphological similarity between the filaments and the bars seen in the radio and in extinctioncorrected line images indicates that they are also associated with the folds in the ionization front seen edge-on. Most probably, the $10 \mu \mathrm{m}$ emission is dominated by PAH emission in the PDR. Sloan et al. (1996) have shown that the $11.3 \mu \mathrm{m}$ $\mathrm{PAH}$ feature, produced by the aromatic $\mathrm{C}-\mathrm{H}$ bonds when a grain is transiently heated through the absorption of a UV photon, dominates the mid-IR spectrum of the Orion bar.

Similarly, the great arcs visible at $10 \mu \mathrm{m}$ approximately $45^{\prime \prime}$ south of the Trapezium (Fig. 4) may result from the advance of the ionization front between the dense and clumpy medium of the surrounding molecular cloud.

\subsection{The Trapezium Region}

The complex arclike morphology of the mid-IR emission in the vicinity of $\theta^{1}$ Ori $C$ (Fig. 5) has clear optical counterparts in recombination lines, first detected by McCullough et al. (1995). Using [O III] and $\mathrm{H} \alpha$ line images taken with the HST, Bally et al. (1998) found that similar arcs are present around many proplyds within $30^{\prime \prime}$ from $\theta^{1}$ Ori C. Bally et al. (2000) also found arcs in the outskirts of the Orion Nebula, especially in the LL Ori region (which remains outside of our imaged field). Hayward et al. (1994) first suggested the arcs are the bow shocks produced by the interaction of the fast $\left(\simeq 500 \mathrm{~km} \mathrm{~s}^{-1}\right)$ wind from $\theta^{1}$ Ori $\mathrm{C}$ with the slow $(\simeq 10-50 \mathrm{~km}$ $\mathrm{s}^{-1}$ ) photoevaporated flows emerging from the ionization front at the proplyd surfaces. This interpretation is supported by proper-motion (Bally et al. 2000) and spectroscopic (Henney et al. 2002) measurements, showing that these are stationary structures. The wind-wind interaction originating the arcs has been studied analytically by several authors (e.g., Cantó et al. 1996 and references therein) and numerically by García-Arredondo et al. (2001). There are in fact two basic configurations: the interaction of two spherical winds, of interest in our case, and the interaction of a spherical wind with a plane-parallel flow. The latter is probably the case in the outskirts of the Orion Nebula, where the wind-wind interaction is occurring between $\mathrm{T}$ Tauri winds and the plasma flowing from the main ionization front (Bally et al. 2000).

The analytical analysis of Cantó et al. (1996), which leads to an exact solution of the wind-wind interaction within the "thin shell" approximation, provides an approximate solution that can be easily compared with observations. The ratio of the wind momenta,

$$
\beta=\frac{\dot{M}_{1} v_{1}}{\dot{M}_{2} v_{2}},
$$

where $\dot{M}$ and $v$ are, respectively, the mass-loss rate and the terminal velocity of the two winds (represented by subscripts), governs both the location of the stagnation point and the shape of the arcs. The ratio between the distance from the stagnation point to the source inside the arc, $R_{0}$, and the separation of the two outflow sources, $D$, is given by

$$
\frac{R_{0}}{D}=\frac{\beta^{1 / 2}}{1+\beta^{1 / 2}} .
$$

The shape of the bow shock is characterized by the asymptotic angle of the tail, $\theta_{\infty}$, given by

$$
\theta_{\infty}-\tan \theta_{\infty}=\frac{\pi}{1-\beta}
$$

If the bow shock axis is tilted with respect to the line of sight by an angle $\theta_{i}$, the projection effect increases both the apparent $R_{0} / D$ ratio and the apparent asymptotic angle. For large tilt angles, the entire back side of the bow shock becomes visible and the characteristic arclike geometry disappears. In these cases, the bow shock will look like a diffuse source in the vicinity of the central star.

Whereas the ratio $R_{0} / D$ can be easily obtained from the data, it is difficult to estimate the asymptotic angle, since the surface brightness of the arcs decreases in the tail region. For this reason, we characterize the shape of the bow shock through the apparent distance, $R_{90}$, between the central star and the arc at $90^{\circ}$ elongation from the direction of $\theta^{1}$ Ori C. It turns out that different combinations of $\beta$ and $\theta_{i}$ generate morphologies with ratios $R_{0} / D$ and $R_{90} / D$ that vary within a relatively narrow range. This variation is shown in Figure 13, where we have plotted the combinations obtained for different values of $\beta$ and $\theta_{i}$. The bow shock morphology, limited to the cases with $\beta=$ $0.1,0.04$, and 0.004 and $\theta_{i}=30^{\circ}$, is also shown to visualize the change of shape along the region.

In Figure 13, we also plot the values of $R_{0} / D$ and $R_{90} / D$ measured for the Ney-Allen Nebula and the five LV arcs. In the case of LV 1, LV 4, and possibly LV 5, the parameters are compatible with the thin shell model. However, in the other three cases, namely, LV 2, LV 3, and the Ney-Allen Nebula, there is a clear disagreement. In correspondence to the observed values of $R_{0} / D$, the values of $R_{90} / D$ should be much larger, because if the two winds carry a similar momentum $(\beta \simeq 1)$ and the stagnation point moves closer to $\theta^{1}$ Ori $C$, the bow shock is expected to become more and more open. Instead, the three arcs with stagnation points closer to $\theta^{1}$ Ori $\mathrm{C}$ show a roughly circular shape, with $R_{0} \simeq R_{90}$.

The intrinsic limitations of the algebraic solution of Cantó et al. (1996) based on the thin shell approximation cannot explain this anomaly. García-Arredondo et al. (2001) have shown that, despite the simplifying assumption of stationary flow and momentum conservation, the analytic treatment nicely matches their detailed hydrodynamic simulation. A possible explanation for the disagreement may better be provided by the detailed morphology of the arcs. The LV 3 arc, in particular, is clearly asymmetric with respect to the direction to $\theta^{1}$ Ori C. It becomes narrower and brighter to the south in the direction of SC 3, with a secondary maximum evident to the northeast at $\simeq 90^{\circ}$ from the $\theta^{1}$ Ori $\mathrm{C}$ direction. The asymmetry indicates that other dynamical effects not accounted for by the standard models are taking place. A relative motion of LV 3 relative to $\theta^{1}$ Ori $\mathrm{C}$ would break the cylindrical symmetry assumed by simple windwind models. A rather modest proper motion, on the order of a few kilometers per second, would be enough to add a significant tangential component to the speed of the photoevaporated flow, which is on the order of the sound speed in a typical ionized gas, $c_{s} \simeq 10 \mathrm{~km} \mathrm{~s}^{-1}$. Other dynamical effects such as the interaction with the collimated mass outflows produced by the central LV stars may also locally affect the morphology and surface brightness of the arcs, leading to inaccurate estimates of the opening angle.

The bright arcs of the Ney-Allen Nebula are peculiar also from another point of view. Let us compare the six brightness 


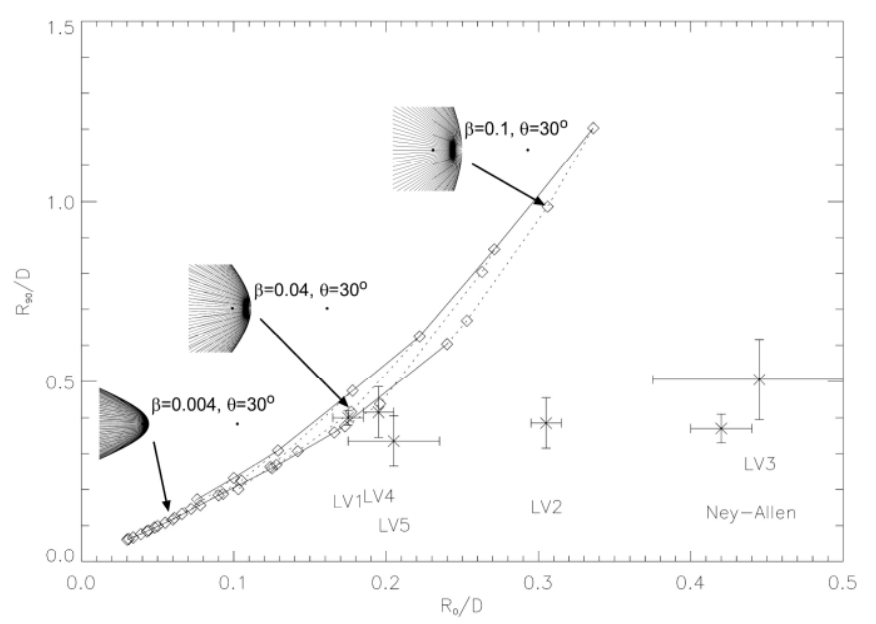

Fig. 13.- - Locus of the morphological parameters $R_{0} / D$ and $R_{90} / D$ describing the Trapezium arcs. Open diamonds refer, from left to right, to $\beta=0.001$, $0.002,0.004,0.01,0.02$, and 0.1 and increase from the bottom to the top from $\theta_{i}=0^{\circ}$ up to the maximum apparent tilt angle in steps of $15^{\circ}$. The location of the arcs in the vicinity of $\theta^{1}$ Ori $\mathrm{C}$ is also shown, with the observational uncertainties.

profiles along the cuts joining $\theta^{1}$ Ori $\mathrm{C}$ to $\theta^{1}$ Ori $\mathrm{D}$ and the LV 1-LV 5 sources (Fig. 14). The LV 1, LV 3, and LV 4 arcs show, on the side facing $\theta^{1}$ Ori $\mathrm{C}$, brightness distributions at 10 and $20 \mu \mathrm{m}$ very similar to each other. The LV 2 and LV 5 arcs, on the other hand, are surrounded by diffuse luminosity, mostly originated by the Ney-Allen Nebula and by the LV 4 arc, and their 10 and $20 \mu \mathrm{m}$ profiles cannot be easily compared. The position of their peaks, however, is the same at both wavelengths, suggesting that also for them the 10 and $20 \mu \mathrm{m}$ emission may be spatially coincident. The fact that the LV 1-LV 5 arcs show a similar morphology at both wavelengths supports our previous hypothesis that the thin shell is a good approximation and that the discrepancies between models and observations can be ascribed to relative motions.

The case of the brightest arc (the Ney-Allen Nebula), however, is different. Figure 14 (top left) shows that the side facing $\theta^{1}$ Ori $\mathrm{C}$ does not show a sharp edge at 10 or at $20 \mu \mathrm{m}$. Moreover, the $20 \mu \mathrm{m}$ emission is more extended in the direction of $\theta^{1}$ Ori $\mathrm{C}$, indicating that a colder dust component becomes brighter at larger distances from the central star. The intensity profiles therefore suggest that (1) the emission is not concentrated in a thin shell, and (2) the UV flux from $\theta^{1}$ Ori C is not the dominant heating source. Departures from the thin shell approximation have theoretical ground. In particular, García-Arredondo et al. (2001) have shown that if the stellar wind passes through an internal reverse shock, the wind-wind interaction takes place in a subsonic regime. In this case, a turbulent mixing layer develops at the interface between the two winds, and filaments and "fingers" are produced by the entrainment of the fast wind within the dense subsonic material. Instead of the sharp decay typical of a thin shell, the drop of surface brightness will appear in this case more gradual and extended. Among the sources in the vicinity of $\theta^{1}$ Ori $C$, this scenario applies naturally, and exclusively, to the Ney-Allen Nebula, as it is associated to the B0 V star $\theta^{1}$ Ori $D$. This hot star originates a substantial stellar wind, and the formation of a internal reverse shock where the gas flows enter a subsonic regime is a natural outcome in the early adiabatic phase of expansion (Castor et al. 1975; Weaver et al. 1977).

The main peculiarity of the Ney-Allen Nebula, however, is that it contains in an extremely harsh environment a considerable amount of dust, estimated between $\simeq 2 \times 10^{27} \mathrm{~g}$ if $T_{d}=200 \mathrm{~K}$ and $\simeq 1 \times 10^{26} \mathrm{~g}$ if $T_{d}=350 \mathrm{~K}$ (Hayward 1994). Proplyds in Orion are supported by the photoevaporation of circumstellar disks around low-mass pre-main-sequence stars (Churchwell et al. 1987; Meaburn 1988; Henney \& Arthur 1998; Johnstone et al. 1998; Henney \& O'Dell 1999). In analogy, it is natural to think of a massive circumstellar disk around $\theta^{1}$ Ori $\mathrm{D}$ as the most natural dust reservoir for the Ney-Allen Nebula. The presence of a massive circumstellar disk is compatible with the standard view that high-mass stars reach the main sequence while they are still in their protostellar accretion phase (Stahler et al. 2000). In this scenario, it is important to notice that $\theta^{1}$ Ori $\mathrm{D}$ is the only star of the Trapezium without a binary or multiple companion, down to a resolution limit $\lambda / D=57$ and 76 mas in the $H$ and $K$ bands, respectively (Preibisch et al. 1999). Conversely, no other Trapezium stars seem to be associated with excess mid-IR emission. This result may therefore indicate that for OB stars the presence of low-mass companions is not compatible with substantial circumstellar disks and vice versa.

Concerning the SC 3 source, i.e., the bright extended blob in the immediate vicinity of $\theta^{1}$ Ori $\mathrm{C}$, we have discussed in a previous paper (Robberto et al. 2002) its apparent morphological anomaly, as it appears undisturbed by the powerful $\theta^{1}$ Ori $\mathrm{C}$ wind despite having the shortest projected distance from $\theta^{1}$ Ori C. On the basis of its high mid-IR brightness, we have proposed that it may represent a disk on the far side of the nebula, oriented face-on with respect to both $\theta^{1}$ Ori $\mathrm{C}$ and the Earth. High spatial resolution mid-IR observations, possibly with interferometric techniques, should easily resolve the radial distribution of its bright mid-IR emission and allow us to clarify the nature of this most enigmatic object.

\subsection{The BN/KL Complex}

\subsubsection{Main Physical Parameters}

The ubiquity and strength of the silicate absorption feature in the $10 \mu \mathrm{m}$ region indicates that foreground absorption plays a dominant role in shaping the observed morphology of the BN/ KL complex. As a baseline, one can therefore assume a twocomponent model (Aitken et al. 1981) in which a background blackbody source is shielded by a foreground absorbing medium too cold to contribute significantly to the mid-IR emission. This assumption allows us to readily obtain a map of the foreground opacity from the line-to-continuum ratio at $9.8 \mu \mathrm{m}$. In a first-order approximation, the continuum can be derived from the interpolation at $9.8 \mu \mathrm{m}$ of the fluxes measured at 7.7 and $12.4 \mu \mathrm{m}$, whereas for the depth of the silicate feature we interpolate between the fluxes measured at 8.7 and $10.2 \mu \mathrm{m}$ to avoid the secondary maximum seen in the $9.6 \mu \mathrm{m}$ filter. The natural logarithm of the absorption-line strength is the optical depth and is presented in Figure 15. The optical depth in the main central region increases from west to east across the entire field, peaking in the immediate vicinity of the dark lane. It reaches the maximum value at the IRc2 position, $\tau_{9.8}=3.37$ (unless otherwise specified, all values are per pixel, i.e., averages over an area $\simeq 0$ "' 06 ), corresponding to $A_{9.8}=3.66$. This is $\simeq 20 \%$ lower than the value reported by Gezari et al. (1998). The drop of extinction coinciding with the dark lane is due to the absence of flux sufficiently high to allow for a meaningful estimate of the extinction and must therefore be regarded as a selection effect.

The map of the peak optical depth allows us to correct all images for the foreground extinction. There are several possible 

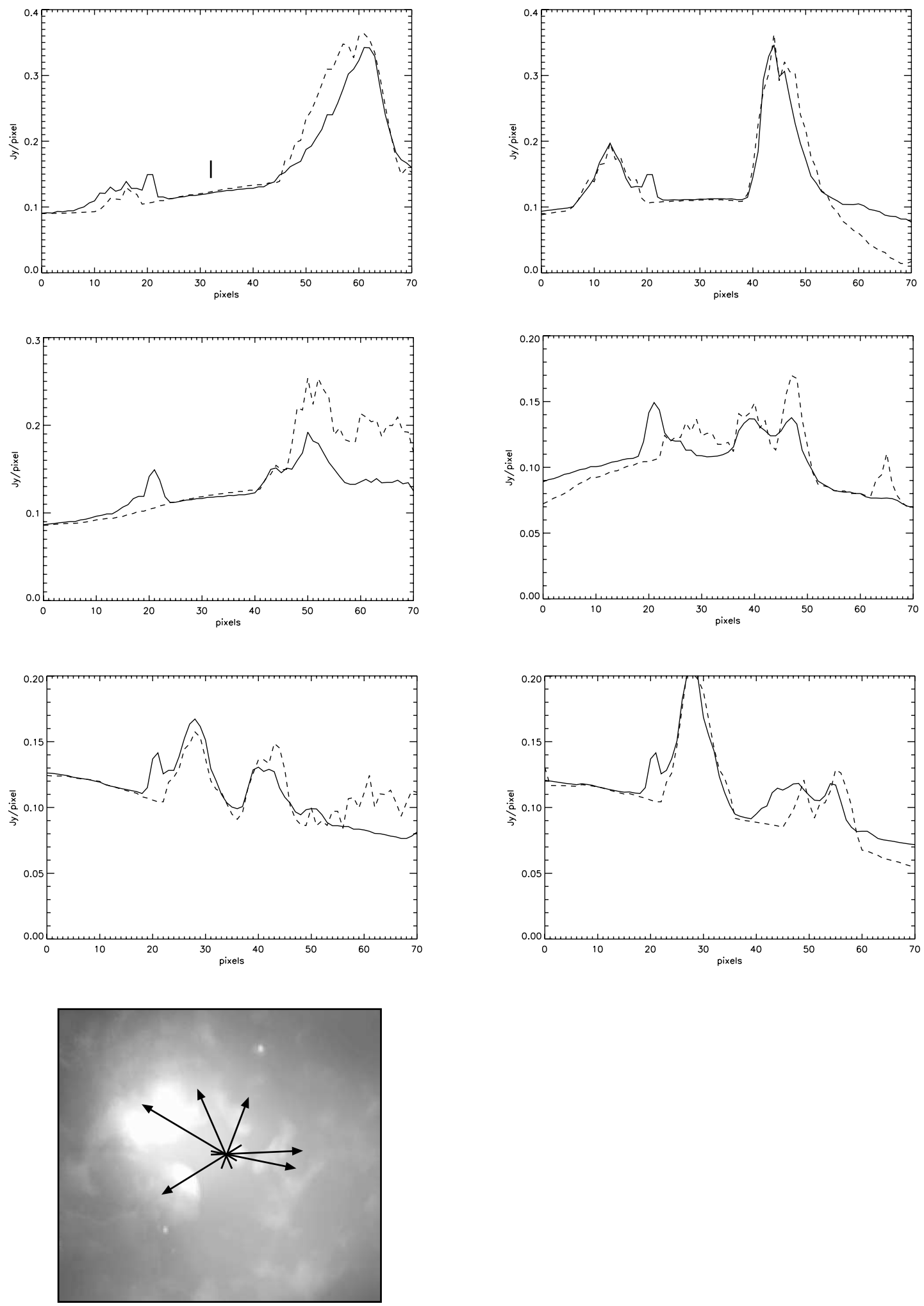

FIG. 14.-Brightness profiles along the cuts joining $\theta^{1}$ Ori $\mathrm{C}$ to $\theta^{1}$ Ori $\mathrm{D}$ and the LV 1-LV 5 sources. Solid line: $10 \mu \mathrm{m}$ emission; dashed line: $20 \mu \mathrm{m}$ emission, scaled for comparison.

choices for the spectral profile of the silicate feature. We adopt here the "Orion Trapezium" emissivity (Forrest et al. 1975), as updated by the spectroscopic observations of Hanner et al. (1995). We average the Hanner et al. emissivity over the (cold) filter transmission profiles provided by the manufacturer. We also include the nonsilicate extinction, which we parameterize in terms of the visual extinction, $A_{V}$, using a fit to the extinction data of Rieke \& Lebofsky (1985), $A_{\lambda}=$ $0.381 A_{V} \lambda^{-1.652}$. Following Rieke \& Lebofsky, we weight the two contributions by assigning to the silicate extinction at the $9.8 \mu \mathrm{m}$ peak a $90 \%$ fraction of the total extinction at that wavelength. 


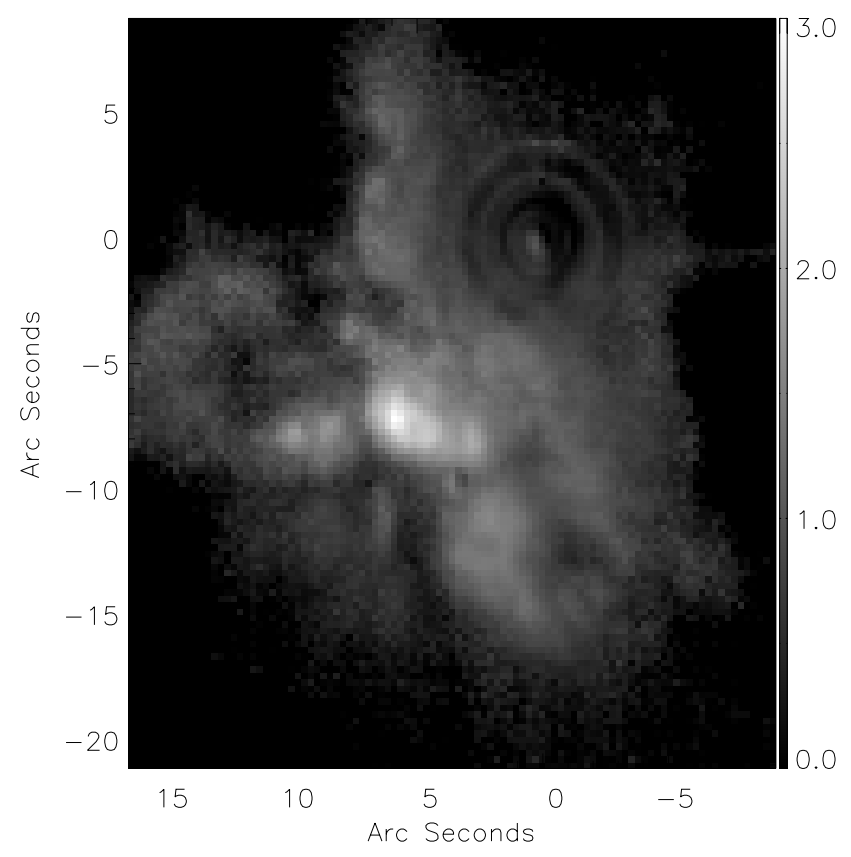

Fig. 15.-Map of the optical depth of the BN/KL complex at $9.6 \mu \mathrm{m}$.

The morphology of the $\mathrm{BN} / \mathrm{KL}$ complex after extinction correction for all six silicate filters is shown in Figure 16. As expected, the changes with respect to the unreddened images (Fig. 7) are stronger where the silicate absorption feature is more prominent. In particular, at 9.8 and $10.2 \mu \mathrm{m}$, the IRc2 object recovers its rank of second brightest source at all wavelengths after the BN peak. In Figure 17, we zoom in on the IRc2 region, comparing for all six silicate filters the morphology before and after extinction correction. The most striking result is the appearance of a point source just to the south of the IRc2 peak. It was visible in the raw $7.7 \mu \mathrm{m}$ images, but now becomes evident also in the dereddened 9.6 and $10.2 \mu \mathrm{m}$ images. Its position is coincident with knot IRc2-A of Dougados et al. (1993) and lies $\simeq 0$ ".5 north of radio source I of Menten \& Reid (1995), which remains undetected. The fact that in the dereddened images the mid-IR peak is visible at $7.7 \mu \mathrm{m}$, invisible at $8.7 \mu \mathrm{m}$, and visible again in the dereddened 9.6 and $10.2 \mu \mathrm{m}$ images may be due to a combination of imperfect extinction correction, intrinsic silicate emission, and loss of resolution at the longest wavelengths.

From the dereddened images, we derive the distribution of the $10 \mu \mathrm{m}$ color temperature of the underlying emission by fitting, pixel by pixel, a blackbody curve to all six silicate filters. The result is shown in Figure 18. The temperature across the $\mathrm{BN} / \mathrm{KL}$ complex typically ranges between 180 and $300 \mathrm{~K}$, with maxima at the $\mathrm{BN}$ peak with $T_{\mathrm{col}} \simeq 1000 \mathrm{~K}$ and at the IRc2 position with $T_{\text {col }} \simeq 420 \mathrm{~K}$. The BN temperature is certainly overestimated. Since BN is unresolved and our images are diffraction-limited, the encircled energy decreases with wavelength. The dilution of flux over a larger number of pixels causes an apparent increase of the color temperature at the peak. Concerning IRc2, the warm dust appears mostly elongated in the northeastern-southwestern direction, i.e., in a direction parallel to the dark lane, and peaks $\simeq 0$ ". 5 to the southeast of source I. This effect is shown in Figure 18, in which we superpose the contours of the dereddened $7.7 \mu \mathrm{m}$ image at 1.5 and $3.0 \mathrm{Jy}$ $\operatorname{arcsec}^{-2}$ flux levels.

The bolometric luminosity can finally be estimated from the $L=\sigma T_{\mathrm{col}}^{4}\left(4 \pi R^{2}\right)$ relation, where $R$ is the radius corresponding to one resolution element $\left(00^{\prime \prime} 26 \simeq 120 \mathrm{AU}\right.$ at the distance of the Orion Nebula). We do not show its spatial distribution, which is very similar to Figure 18 with an even more prominent role of IRc2 and BN.

In Table 5, we list the values of the three parameters we have derived - optical depth at $9.6 \mu \mathrm{m}$, color temperature in the 7.7-12.4 $\mu \mathrm{m}$ band, and total luminosity - for the sources with photometry presented in Table 4 . The values of $\tau_{9.6}$ and $T_{\text {col }}$ are averages over a circular aperture of $1 \operatorname{arcsec}^{2}$ area, whereas the luminosity, $L$, is integrated over the same area. Table 5 also reports the temperatures and luminosities derived from the dereddened 10 and $20 \mu \mathrm{m}$ broadband filters, having assumed at $20 \mu \mathrm{m}$ the interstellar extinction law of Mathis (1990). The color temperature between these two wavelengths (125-140 K) is typically $80 \mathrm{~K}$ lower than that derived from the silicate filters $(200-230 \mathrm{~K})$. The discrepancies of up to a factor of 10 in the estimated luminosities reflect these differences.

The elementary technique we have used so far, based on a crude estimate of the extinction, still provides maps of the spatial variations that are quite robust, since the data have been taken all at the same time and calibration errors are systematic. In the next section we discuss a more refined model to investigate further the characteristics of the mid-IR emission of the $\mathrm{BN} / \mathrm{KL}$ complex.

\subsubsection{Silicate Absorption Feature}

The two-component model has been successfully used in the past to model the $10 \mu \mathrm{m}$ spectrum of the main BN peak (Aitken et al. 1981, 1985). Figure 19 compares our broadband photometry of BN calculated over a synthetic aperture of 5".2 diameter with the spectrophotometric data of Aitken et al. (1985) and the synthetic photometry of Gezari et al. (1998). The agreement between different data sets is good, considering that the broadband values have not been color-corrected and the differences in aperture diameter between different authors. The solid and dotted lines represent the locus of a blackbody curve at $T_{\text {col }}$ before and after reddening by an optical depth $\tau_{9.8}$ to fit our photometry. We use the Trapezium emissivity of Hanner et al. (1995) with the additional $\lambda^{-1.65}$ continuum discussed above. The best fit to our data has $T_{\text {col }}=373 \mathrm{~K}$ and $\tau_{9.8}=1.37$, with a filling factor $\mathrm{FF}=5.41 \times 10^{-4}$ to normalize the absolute flux levels. From the filling factor, we derive a photospheric radius $R_{\mathrm{BN}}=27.2 \mathrm{AU}$ at $450 \mathrm{pc}$, and the resulting luminosity is $L_{\mathrm{BN}}=4 \pi R_{\mathrm{BN}}^{2}\left(\sigma T_{\mathrm{col}}^{4}\right)=600 L_{\odot}$. For comparison, Aitken et al. (1981) derive $T_{\text {col }}=336 \mathrm{~K}$ and $\tau_{9.8}=1.54$, whereas the total luminosity of $\mathrm{BN}$ is $L_{\mathrm{BN}} \simeq(1-2) \times 10^{4} L_{\odot}$ (Scoville et al. 1983).

It is known that, besides the BN peak, the two-component fit does not account for the detailed shape of the $10 \mu \mathrm{m}$ spectrum of the other compact sources of the BN/KL complex (Aitken et al. 1985). The 8-13 $\mu \mathrm{m}$ absorption feature appears to change across the complex, both in depth and in shape (Aitken et al. 1981). These variations reflect different physical conditions in the absorbing medium. Downes et al. (1981) and Werner et al. (1983) first indicated that most of the IRc sources discovered in the BN/KL complex are not self-luminous, but rather extended clumps of diffuse emission. Genzel \& Stutzki (1989) indicated that the $\mathrm{BN} / \mathrm{KL}$ complex is dominated by the radiative transport of IR radiation in a highly nonuniform medium. A further complication may arise from the fact that at shorter wavelengths scattering becomes important. Werner et al. (1983) were the first to report that at $4.8 \mu \mathrm{m}$ the $\mathrm{BN} / \mathrm{KL}$ complex is predominantly a reflection nebula, but since the albedo of astronomical silicates drops by 2 orders of magnitude between 

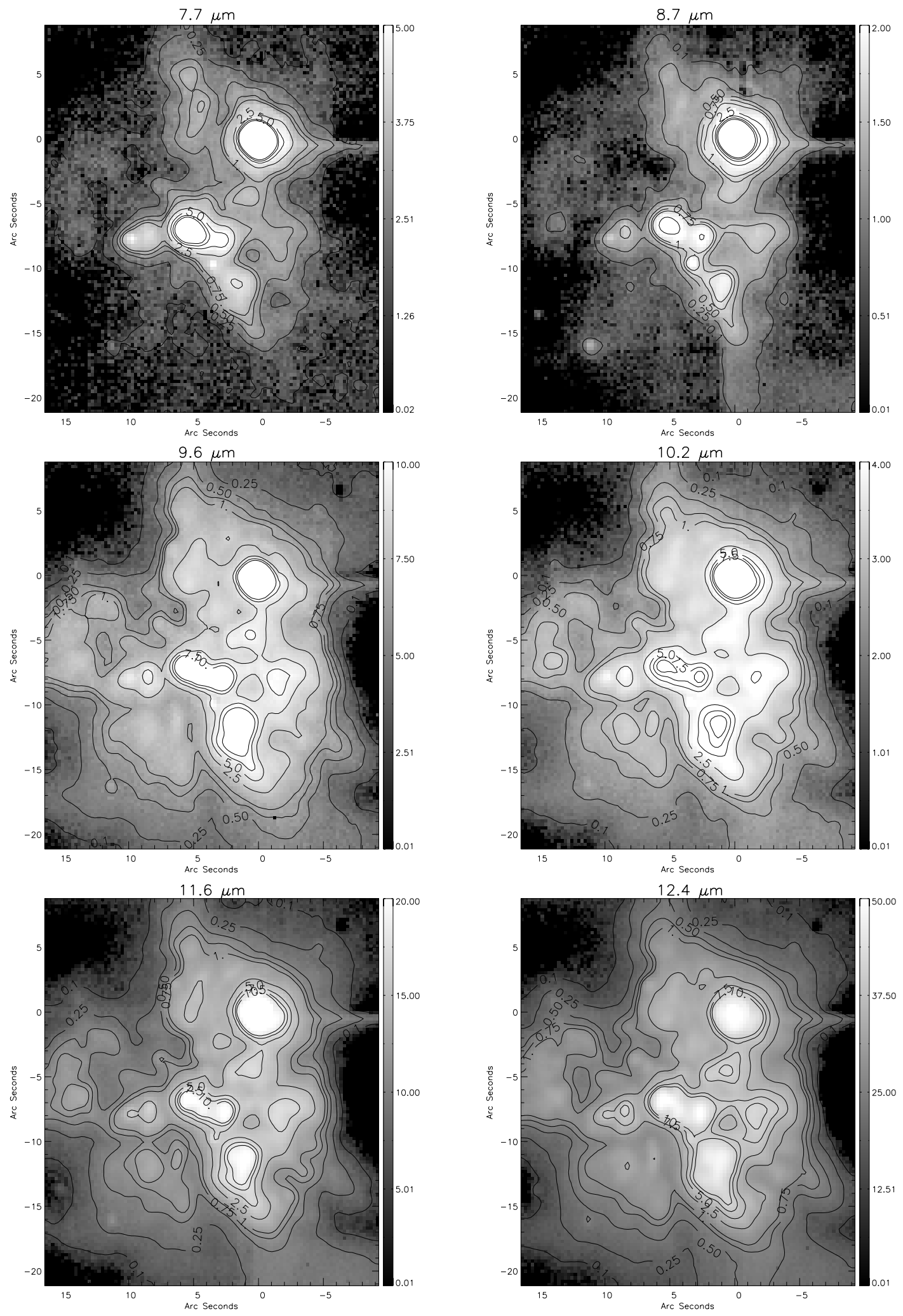

FIG. 16.- Same as Fig. 7, but after reddening correction.

4.5 and $8 \mu \mathrm{m}$ (Draine 1985), one can still assume that scattering is negligible at mid-IR wavelengths. In this case, the basic two-component model can be modified to account for the following effects:

1. The background emission is normally optically thin. Only for a few sources, like BN and IRc2, can the background emis- sion be represented by a stellar photosphere multiplied by a proper filling factor.

2. The presence of foreground absorbing medium (as in the two-component model).

3. A third component, optically thin, needed to account for silicate emission at $9.6 \mu \mathrm{m}$. 

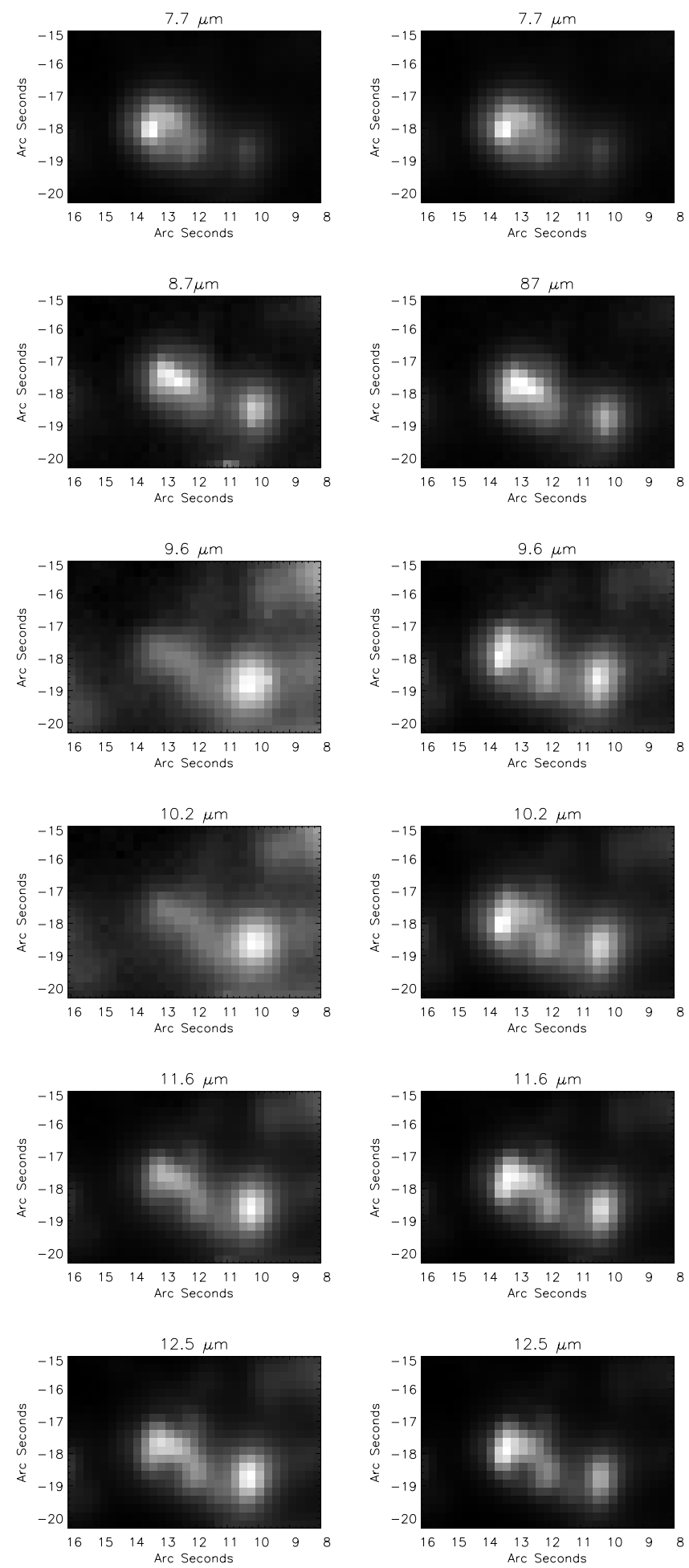

FIG. 17.-Comparison of the morphology of the IRc2/IRc7 region before and after reddening correction for the six silicate filters.

In a slab geometry, these three factors are described by the equation

$$
\begin{aligned}
F_{\nu} & =\operatorname{FF} B_{\nu}\left(T_{b}\right)\left(1-\exp ^{-\tau_{b}(\nu)}\right) \exp ^{-\tau_{a}(\nu)} \\
& +B_{\nu}\left(T_{s}\right)\left(1-\exp ^{-\tau_{s}(\nu)}\right)
\end{aligned}
$$

Here FF $\leq 1$ and $T_{b}$ are the filling factor and temperature of a background medium with optical depth, $\tau_{b}$. For a photosphere (e.g., the BN peak), it is $\left(1-\exp ^{-\tau_{b}}\right) \simeq 1$. In addition, $\tau_{a}$ is the optical depth of the cold foreground absorbing material, which we assume is mostly located in the vicinity of the background sources (i.e., part of the Orion molecular cloud), and $T_{e}$ and $\tau_{e}$ are the temperature and optical depth of the foreground gas responsible for the silicate emission. We locate this third component in the foreground of the main absorbing layer, assuming that it physically corresponds to warm dust heated in the foreground cavity, i.e., contained in the optically visible Orion Nebula.

Equation (4) contains six free parameters, and the choice of different emissivity laws allows for some extra freedom. For this exploration, we adopt the silicate emissivity law of Jäger et al. (1994), which provides excellent agreement with the spectra of massive young stellar objects and the Ney-Allen Nebula in what concerns the position of the $10 \mu \mathrm{m}$ peak and has a relatively narrow width that may provide the best agreement with the most recent measures of the Trapezium (see below). Figure 20 (left) shows the best fit to the flux (averaged over 4 pixels) at the IRc2 peak. To allow the numerical routine to converge, we had to reduce the degree of complexity of our system. Thus, the dotted line represents the fit to the 7.7, 8.7, 10.2, 11.6, and $12.4 \mu \mathrm{m}$ bands, i.e., all the silicate filters except the $9.6 \mu \mathrm{m}$ one, provided by the first term at the right-hand side of equation (4), having assumed $\mathrm{FF}=1$. We obtain $T_{b}=710 \mathrm{~K}, \tau_{b}=0.0019$, and $\tau_{a}=$ 21.6. The dashed line represents the contribution from the second term at the right-hand side, having assumed ad hoc $T_{s}=$ $300 \mathrm{~K}$ and $\tau_{s}=1.5 \times 10^{-3}$ to approximately match the photometry in the central part of the silicate feature. The thick solid line represents the sum of the two contributions. Figure 20 (left) shows that the $20 \mu \mathrm{m}$ point cannot be matched by a single blackbody temperature because of the strength of the secondary silicate absorption peak at $18 \mu \mathrm{m}$. At $10 \mu \mathrm{m}$, the emissivity law of Jäger et al. is too broad to match the 8.7 and $10.2 \mu \mathrm{m}$ points, but the addition of the third component in emission provides a reasonable fit, including the $9.6 \mu \mathrm{m}$ point. A detailed look shows that the emissivity law of Jäger et al. (1994) is still too broad to reproduce at the same time the $9.6 \mu \mathrm{m}$ peak and the adjacent 8.7 and $10.2 \mu \mathrm{m}$ points. The finite filter bandpass, together with the presence of a range of temperatures instead of the assumption of a single $300 \mathrm{~K}$ blackbody, exacerbates the problem, as both effects tend to reduce the observed sharpness of the feature. To illustrate the effect of the bandpass, we have convolved the sum of the two contributions with a $\Delta \lambda=1 \mu \mathrm{m}$ rectangular bandpass. The result is presented as a thin solid line in Figure 20 (left). Similar results are found for other sources, and in Figure 20 (right) we show as an example the case of IRc7, where we find $T_{b}=232 \mathrm{~K}, \tau_{b}=$ $0.04, \tau_{a}=14.2$, and $\tau_{s}=0.0035$.

The value $\tau_{9.8} \simeq 22$ that we have found for IRc2 corresponds to $A_{v} \simeq 400$ and is compatible with the extreme value of the optical depth $\tau \gtrsim 300$ at $8.0 \mu \mathrm{m}$ at the near ammonia core estimated by Greenhill et al. (2004). A general emissivity law with a narrower peak would provide higher values of column density. On the other hand, there is no reason to assume a priori that the emissivity is the same for the three components or in general across the region. There is evidence that, for young stellar objects and star-forming regions, the $10 \mu \mathrm{m}$ silicate feature depends on the environment because of the interplay between dust column density, cosmic ray irradiation, and grain coagulation (Bowey et al. 2003). In particular, the shape of the canonical "Trapezium emissivity" of Forrest et al. (1975), Roche \& Aitken (1984), and Whittet et al. (1988), generally assumed as a reference for molecular clouds and used to 


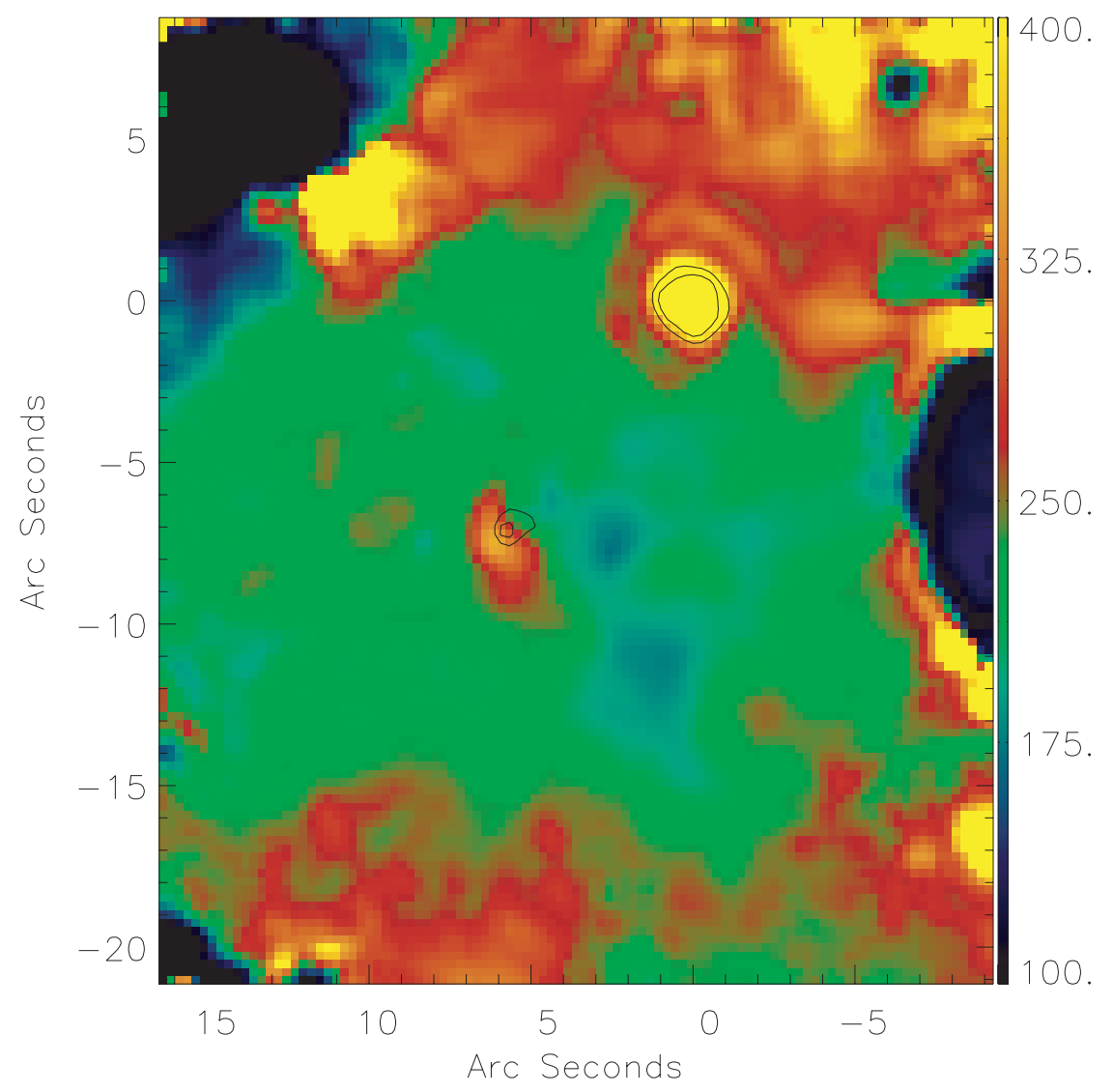

Fig. 18.-Map of the $10 \mu \mathrm{m}$ color temperature.

constrain the optical constants of the popular Draine \& Lee (1984) model, shows a dependence on the spatial scale. Unpublished arcsecond-resolution spectra of the Trapezium obtained by T. Hayward (Bowey 1998) show that the peak of the $10 \mu \mathrm{m}$ feature is significantly narrower $(0.5-0.6 \mu \mathrm{m})$ than that reported by Forrest et al. (1975) and Hanner et al. (1995), these last authors with a 9.4 beam (beam size is unquoted in the Forrest et al. paper). Different broadening may be due to grain size effects or to changes in the grain structure or optical properties (see Jäger et al. 1994 for a discussion). Bowey (1998) suggests that measures made with large aperture, sampling a wide range of dust temperatures, may also cause a broadening of the $10 \mu \mathrm{m}$ silicate feature, at least in the case of the Trapezium. If the optically thin emission is due to dust grains exposed to UV radiation, the grain structure may be affected in the direction of a predominantly crystalline composition. Amorphous silicates may be converted to crystalline minerals when annealed (heated) in vacuum at high temperature (Day \& Donn 1978). The Trapezium grains are also exposed to strong UV radiation, but since they may have been only recently released by the disk surrounding $\theta^{1}$ Ori $\mathrm{D}$, they may still represent a relatively standard mixture of amorphous and crystalline silicates. On the other hand, the dust grains inside the Orion Nebula, and those distributed in front of the $\mathrm{BN}$ region in particular, could have been exposed for a longer time to the nebular UV radiation. It is intriguing to notice that the reddening law in Orion has $R_{V}=A_{v} / E(B-V) \simeq 5.5$, versus the standard value $R_{V}=3.1$ of the interstellar medium. This anomaly could be related to the peculiar evolution of the dust grains in the harsh nebular environment.

\subsubsection{The Role of IRc2 and Source $n$}

IRc2 has been considered for a long time as the mid-IR source more closely associated with a deeply embedded center of activity of the region, e.g., the origin of the spectacular outflow phenomena emerging from the BN/KL complex. Evidence in this direction comes from the position of IRc 2 at the center of (1) an expanding cluster of maser sources with velocity centroid nearly coincident to the LSR velocity of the hot core (Genzel et al. 1981), (2) a well-defined polarization pattern (Aitken et al. 1997; Chrisostomou et al. 2000), and (3) a rich outflow phenomenology observed at near-IR wavelengths (Jones \& Walker 1985). Source I of Menten \& Reid (1995), a compact $\mathrm{H}$ II region approximately $1^{\prime \prime}$ south of IRc2, has been more recently indicated as the actual center of activity of the $\mathrm{BN} / \mathrm{KL}$ complex (see Tan 2004 for a recent review). Whether IRc2 is just a complex of knots heated by source I or, as Greenhill et al. (2004) speculate, a group of individual stars associated with source I in a young multiple system, remains unclear. An intermediate case could also be considered, with source I and the IRc2-A peak representing deeply embedded individual sources and the other IRc2 knots being diffuse sources heated mostly by the IRc2-A peak.

Source $n$, whose radio emission has been resolved into a bipolar nebula by Menten \& Reid (1995), has also recently gained a prominent role. It is only $3^{\prime \prime}$ away from IRc2, and it lies exactly at the center of expansion of the $\mathrm{H}_{2} \mathrm{O}$ masers of Genzel et al. (1981), within the narrow 2".0 $\times 0.5$ strip where Gaume et al. (1998) locate their "shell" masers. Both Shuping et al. (2004) and Greenhill et al. (2004) have been able to resolve this 
TABLE 5

Physical Parameters of the IRc Sources in the BN/KL Complex

\begin{tabular}{|c|c|c|c|c|c|}
\hline \multirow[b]{2}{*}{ Source Name } & \multirow[b]{2}{*}{$\tau_{9.6}$} & \multicolumn{2}{|c|}{$7.7-12.4 \mu \mathrm{m}$} & \multicolumn{2}{|c|}{$10-20 \mu \mathrm{m}$} \\
\hline & & $\begin{array}{l}T_{\mathrm{col}} \\
(\mathrm{K})\end{array}$ & $\begin{array}{c}L \\
\left(L_{\odot}\right)\end{array}$ & $\begin{array}{l}T_{\mathrm{col}} \\
(\mathrm{K})\end{array}$ & $\begin{array}{c}L \\
\left(L_{\odot}\right)\end{array}$ \\
\hline $\mathrm{BN} \ldots$ & 0.51 & 490 & $9.21 \times 10^{5}$ & 296 & $7.98 \times 10^{4}$ \\
\hline 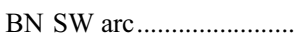 & 0.33 & 288 & $8.48 \times 10^{4}$ & 176 & $8.39 \times 10^{3}$ \\
\hline IR $n$ & 1.30 & 227 & $2.41 \times 10^{4}$ & 149 & $4.37 \times 10^{3}$ \\
\hline 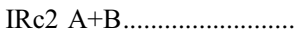 & 2.91 & 308 & $1.14 \times 10^{5}$ & 164 & $6.27 \times 10^{3}$ \\
\hline IRc2 C........ & 2.53 & 258 & $4.49 \times 10^{4}$ & 153 & $4.70 \times 10^{3}$ \\
\hline IRc2 D....... & 2.36 & 219 & $2.11 \times 10^{4}$ & 160 & $5.68 \times 10^{3}$ \\
\hline 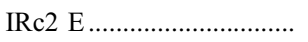 & 1.94 & 208 & $1.63 \times 10^{4}$ & 148 & $4.06 \times 10^{3}$ \\
\hline 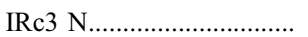 & 1.11 & 204 & $1.46 \times 10^{4}$ & 127 & $2.27 \times 10^{3}$ \\
\hline IRc3 $\mathrm{S} . .$. & 1.18 & 209 & $1.61 \times 10^{4}$ & 128 & $2.35 \times 10^{3}$ \\
\hline IRc4 & 1.53 & 184 & $9.55 \times 10^{3}$ & 138 & $3.12 \times 10^{3}$ \\
\hline IRc5 .......... & 1.32 & 206 & $1.53 \times 10^{4}$ & 126 & $2.15 \times 10^{3}$ \\
\hline IRc6.......... & 1.30 & 210 & $1.62 \times 10^{4}$ & 133 & $2.69 \times 10^{3}$ \\
\hline 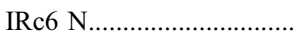 & 0.87 & 223 & $2.09 \times 10^{4}$ & 133 & $2.72 \times 10^{3}$ \\
\hline IRc6 E........ & 1.48 & 206 & $1.52 \times 10^{4}$ & 134 & $2.75 \times 10^{3}$ \\
\hline IRc $7 \ldots \ldots \ldots$ & 2.02 & 198 & $1.14 \times 10^{4}$ & 148 & $4.17 \times 10^{3}$ \\
\hline IRc $8 \ldots \ldots \ldots \ldots$ & 0.79 & 225 & $2.16 \times 10^{4}$ & 119 & $1.69 \times 10^{3}$ \\
\hline IRc11 ......... & 1.73 & 215 & $1.87 \times 10^{4}$ & 144 & $3.69 \times 10^{3}$ \\
\hline IRc12 ........ & 1.79 & 258 & $3.93 \times 10^{4}$ & 140 & $3.34 \times 10^{3}$ \\
\hline IRc13 $\ldots \ldots \ldots$. & 0.84 & 233 & $2.49 \times 10^{4}$ & 118 & $1.66 \times 10^{3}$ \\
\hline 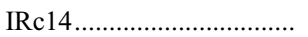 & 0.95 & 223 & $2.11 \times 10^{4}$ & 124 & $2.03 \times 10^{3}$ \\
\hline 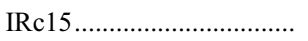 & 1.00 & 222 & $2.06 \times 10^{4}$ & 130 & $2.41 \times 10^{3}$ \\
\hline 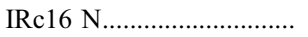 & 1.06 & 238 & $2.70 \times 10^{4}$ & 128 & $2.32 \times 10^{3}$ \\
\hline 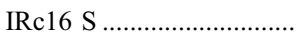 & 1.11 & 227 & $2.25 \times 10^{4}$ & 136 & $2.97 \times 10^{3}$ \\
\hline IRc16 W...... & 0.86 & 233 & $2.47 \times 10^{4}$ & 140 & $3.38 \times 10^{3}$ \\
\hline IRc17 ........... & 0.65 & 247 & $3.16 \times 10^{4}$ & 122 & $1.89 \times 10^{3}$ \\
\hline IRc19........ & 1.02 & 303 & $7.30 \times 10^{4}$ & 131 & $2.48 \times 10^{3}$ \\
\hline $\operatorname{IRc} 20 \ldots \ldots$ & 1.33 & 214 & $1.75 \times 10^{4}$ & 126 & $2.18 \times 10^{3}$ \\
\hline 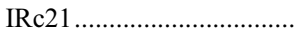 & 1.63 & 221 & $2.03 \times 10^{4}$ & 134 & $2.74 \times 10^{3}$ \\
\hline IRc $22 \ldots \ldots$. & 1.05 & 229 & $2.36 \times 10^{4}$ & 126 & $2.15 \times 10^{3}$ \\
\hline 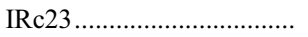 & 1.21 & 239 & $2.80 \times 10^{4}$ & 126 & $2.12 \times 10^{3}$ \\
\hline 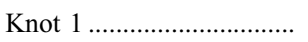 & 0.81 & 240 & $2.83 \times 10^{4}$ & 115 & $1.48 \times 10^{3}$ \\
\hline Knot $2 \ldots \ldots \ldots$ & 0.84 & 246 & $3.12 \times 10^{4}$ & 114 & $1.43 \times 10^{3}$ \\
\hline 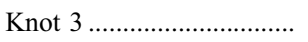 & 0.85 & 245 & $3.06 \times 10^{4}$ & 115 & $1.50 \times 10^{3}$ \\
\hline 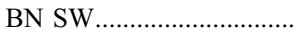 & 0.77 & 234 & $2.53 \times 10^{4}$ & 140 & $3.30 \times 10^{3}$ \\
\hline 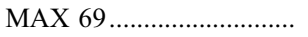 & $\ldots$ & 297 & $6.79 \times 10^{4}$ & 128 & $2.36 \times 10^{3}$ \\
\hline
\end{tabular}

source at $10 \mu \mathrm{m}$ with Keck. It appears slightly elongated in a direction perpendicular to the axis of the bipolar radio source. Shuping et al. (2004) suggest that source $n$ lies at the center of a bipolar cavity excavated by the same outflow responsible for the reach maser phenomenology. The presence of a compact H II region clearly suggests that this is a young early-type star. Greenhill et al. (2004) estimate a luminosity $L \sim 2000 L_{\odot}$ (mid$\mathrm{B}$ type), but also note that the recent detection of hard X-ray emission is unusual for early-type stars.

Gezari et al. (1998) have used mid-IR photometric data similar to ours to downplay the role of IRc2 versus source $n$ as the dominant energy source of the region. In particular, Gezari et al. (1998) estimate for IRc2 a total luminosity $L \simeq 10^{3} L_{\odot}$, approximately 2 orders of magnitude lower than the previous estimates (e.g., Downes et al. 1981; Wynn-Williams et al. 1984). One may question the reliability of a technique that provides values different by orders of magnitudes in front of a rather modest gain in spatial resolution. In fact, these estimates must always be taken with great caution. First, we have seen in the previous paragraphs that different analysis techniques may lead to very different values for the optical depth at $9.8 \mu \mathrm{m}$ $\left(\tau_{9.8}=3.37\right.$ vs. $\left.\tau_{a}=21.6\right)$, even if both use the same data set (in our particular case, both neglect the $9.6 \mu \mathrm{m}$ point). It is also known that the commonly used relation, $L=4 \pi R^{2} \sigma T^{4} \epsilon$, where $R$ and $T$ are the source radius and temperature and $\epsilon$ represents the dust emissivity, may lead to a significant underestimate of the bolometric luminosity if one assumes the IR color temperature as the blackbody temperature of the source (Panagia 1975). A more subtle effect may also be present, since the same relation also assumes that the size of the underlying photosphere does not change with wavelength. In the case of IRc2, however, if the absorbing medium is clumpy or in general inhomogeneous, an unresolved source may have a filling factor that increases with wavelength as the foreground absorbing material becomes more and more transparent. The underlying source will then appear redder, and the estimated luminosity will be lower because of the strong dependence on the color temperature. In particular, a value $A_{V}=60 \mathrm{mag}$ corresponds to $A_{9.7} \simeq 3.4 \mathrm{mag}$ at $9.7 \mu \mathrm{m}$ and to $A_{20}=1.3 \mathrm{mag}$ at $20 \mu \mathrm{m}$, using the Bessell \& Brett (1988) IR reddening law. Thus, IRc2 can be nearly optically thick at $10 \mu \mathrm{m}$ and nearly optically thin at $20 \mu \mathrm{m}$. In these conditions, the mid-IR morphology is determined by a complex combination of thermal emission and variations of foreground extinction. Further multiwavelength observations will need to be coupled with three-dimensional radiative transfer models to disentangle each contribution. 


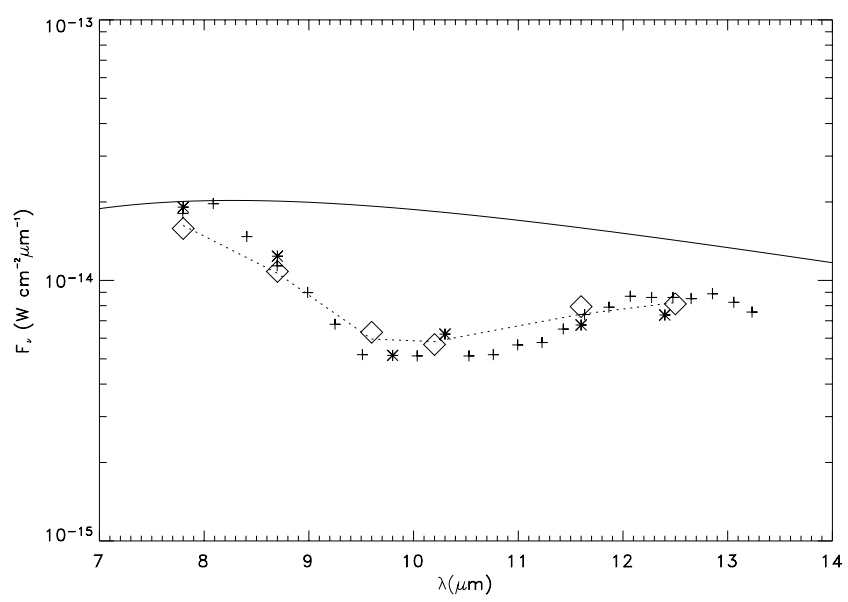

FIG. 19.- Silicate spectrum of BN. Diamonds: this paper; plus signs: spectrophotometry of Aitken et al. (1985); asterisks: broadband data of Gezari et al. (1998). The solid line refers to a blackbody curve at $T_{\mathrm{col}}=373 \mathrm{~K}$. The dotted line joins the blackbody points corresponding to our effective wavelengths after extinction correction with optical depth $\tau_{9.8}=1.37$.

\subsection{OMC-1 South}

The OMC-1 South region is characterized by the rich phenomenology typically associated with massive star formation: warm gas (Ziurys et al. 1981), large dust column densities (Keene et al. 1982), $\mathrm{H}_{2} \mathrm{O}$ masers (Gaume et al. 1998), molecular outflows (Schmid-Burgk et al. 1990; Rodríguez-Franco et al. 1999a), and several optical jets and Herbig-Haro (HH) objects (Bally et al. 2000). With nine mid-IR sources in a less than $1^{\prime} \times 1^{\prime}$ field (Fig. 11), this is a rather dense area, and the scale of the outflows, up to a projected distance of $\simeq 100^{\prime \prime}=$ $0.21 \mathrm{pc}$ in the case of $\mathrm{HH} 203 / 204$, often makes it difficult to unambiguously associate each optical outflow with a well-defined driving source. We have already mentioned the correspondence between the reddest $3.5 \mu \mathrm{m}$ sources, TPSC 1 and TPSC 2 of Lada et al. (2000), and MAX 61 and MAX 64, respectively. A system of outflows that includes HH 529 and HH 269, and possibly HH 528, appears to depart from an "optical outflow source" (named OOS by O'Dell \& Doi 2003a) coincident, within the error ellipse, with both sources. Based on their

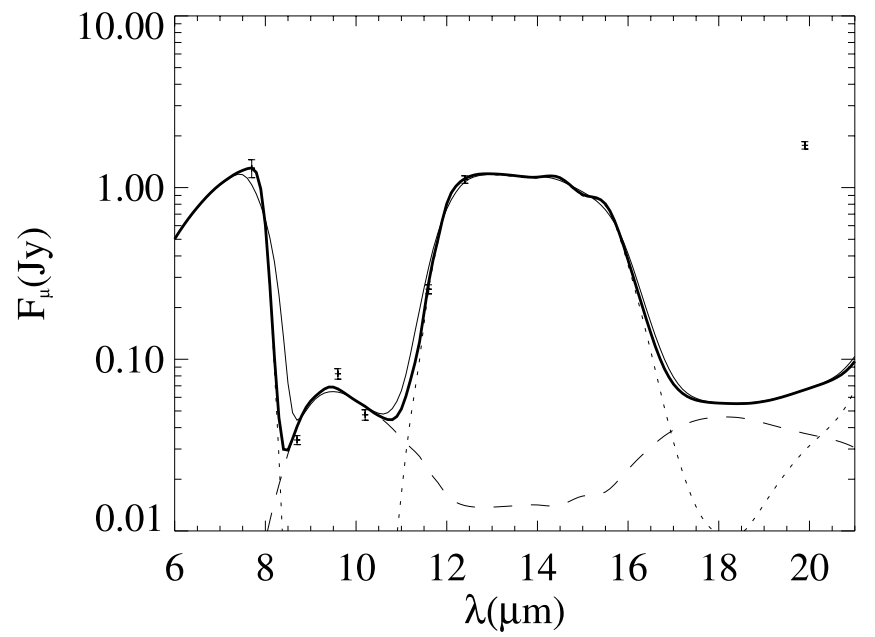

proper motions, O'Dell \& Doi (2003a, 2003b) estimate for the OOS coordinates R.A.(J2000) $=5^{\mathrm{h}} 35^{\mathrm{m}} 14.56$, decl.(J2000) $=$ $-5^{\circ} 23^{\prime} 54^{\prime \prime}$, within $\sim 3^{\prime \prime}$ from the position of MAX 61 and MAX $64 .{ }^{6}$ MAX 61, which is radio source 144-351 of Zapata et al. (2004), is the most viable candidate, since our images (Fig. 4) show two elongated filaments immediately to the east and west of MAX 61 with the same orientation as the HH 529 and HH 269 outflows. These are most probably the jets emerging from the source, with the mid-IR emission due to entrained dust on their outside, along with gas. Doi et al. (2004) combine proper motions with new radial velocities of the outflows to determine that the optical jets originate from a source lying only about $0.02 \mathrm{pc}$ within the PDR. Their relations between proper motion, radial velocities, and the mid-IR emission would provide a self-consistent picture of this activity center, except that both systems of HH objects appear blueshifted and breaking through the ionization front, a rather unusual circumstance that may indicate a more complex scenario. A curvature of the outflow toward our line of sight could result from the interaction of the jet with some stellar wind, originated, e.g., from the Trapezium stars or from the nebular ionization front. This is the hypothesis of Smith et al. (2004). It is clear that if the jet direction can be deflected by wind interaction, the identification of candidate outflow sources on the basis of proper motion and jet/counterjet alignment becomes more uncertain. In fact, Smith et al. (2004) cast doubts about the association of HH 269 with HH 529 and rather suggest that HH 269 is the counterlobe of the HH 203/204 pair, with significant deflection with respect to both the plane of the sky and our vantage point.

Concerning the other prominent optical outflows, both Zapata et al. (2004) and Smith et al. (2004) argue that HH 202 is associated with HH 528 rather than HH 203/204, as suggested by Rosado et al. (2001). Zapata et al. (2004) suggest that this system originates from their radio source 143-353. At this position, we detect an extended knot of $10 \mu \mathrm{m}$ emission shown to be saturated in Figure 4 and not included in our list of 177 point sources. Smith et al. (2004) suggest a different scenario, with the HH 202

\footnotetext{
${ }^{6}$ Zapata et al. (2004) base their comparison with the present paper on a preliminary version of the manuscript. In this final version their sources MAX 58 and MAX 60 are now listed as MAX 61 and MAX 64.
}

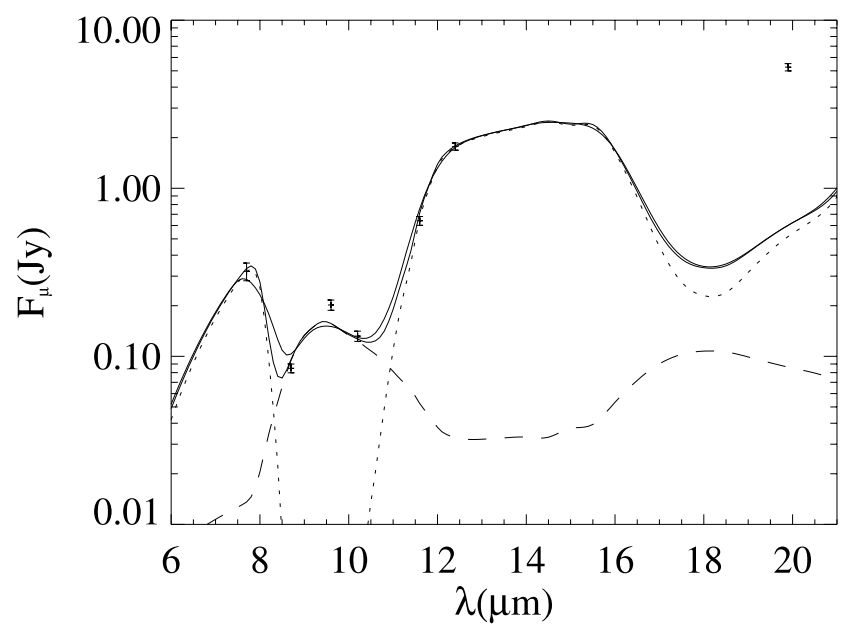

FIG. 20.-Fit to the mid-IR photometry of IRc2 (left) and IRc7 (right) on the basis of eq. (4). Dotted line: First term on the right side of eq. (4), relative to the background source and foreground extinction. Dashed line: Second term on the right side of eq. (4), relative to the optically thin emission of $300 \mathrm{~K}$ dust. Thick solid line: Sum of the two contributions. Thin solid line: Sum of the two contributions convolved with a $1 \mu \mathrm{m}$ rectangular bandpass to reproduce the effect of finite bandwidth. Error bars represent the zero-point errors quoted in $\S 2.2 .3$. 
jet associated to their source 1 (MAX 45), but they also note that neither MAX 61 nor MAX 64 cannot be ruled out.

In what concerns the molecular outflows, the $\lambda=1.3 \mathrm{~mm}$ bolometer maps of Mezger et al. (1990) show a peak of dust continuum brightness, source FIR 4, located at R.A.(J2000) = $5^{\mathrm{h}} 35^{\mathrm{m}} 13.3$, decl.(J2000) $=-5^{\circ} 24^{\prime} 12^{\prime \prime}$.5. Low-velocity molecular outflows depart from the vicinity of FIR 4 (Ziurys et al. 1990; Schmid-Burgk et al. 1990). A second, very young (dynamical age of $\left.\sim 10^{3} \mathrm{yr}\right)$, very fast $\left(\sim 110 \mathrm{~km} \mathrm{~s}^{-1}\right)$, and very compact $(\lesssim 0.16 \mathrm{pc})$ molecular outflow has also been discovered by Rodríguez-Franco et al. (1999a, 1999b). The geometry of the high-velocity gas suggests that its powering source is located $\approx 20^{\prime \prime}$ north of FIR 4 . We detect no IR sources within a $12^{\prime \prime}$ radius from FIR 4; in particular, there is no mid-IR source at the position of the $1.3 \mathrm{~cm}$ source 134-411 of Zapata et al. (2004). At the other position $20^{\prime \prime}$ north, there are three near-IR sources of Hillenbrand \& Carpenter (2000), i.e., HC 190, HC 198, and HC 199, but none of them have been detected at longer wavelengths either by us or by Lada et al. (2000). On the other hand, sources MAX 42 and MAX 43, corresponding to TPSC 16 and TPSC 46 of Lada et al. (2000) and to the radio sources 136-359 and 136-356 of Zapata et al. (2004), are displaced $\sim 5^{\prime \prime}-10^{\prime \prime}$ to the southeast, still in a favorable position with respect to the symmetry point of the $\mathrm{CO}$ outflow. Both show a remarkable $K-L \simeq 3$ color and appear as excellent candidates for the exciting source. The fainter of the two, MAX 42, is better aligned with the symmetry axis of the outflow. The best groundbased near-IR images clearly show that MAX 43 is diffuse. Source MAX 47, corresponding to radio source 139-357 of Zapata et al. (2004), also lies along the axis of the outflow. We have detected this source only at $20 \mu \mathrm{m}$, whereas Smith et al. (2004) have only a $10 \mu \mathrm{m}$ detection. Since there is no near-IR counterpart at this location, this appears to be a deeply embedded source and therefore also represents a good candidate for the source driving the molecular outflow.

The last protostellar candidate of Lada et al. (2000), TPSC 78 , corresponding to source MAX 46, is located near a characteristic feature of the Orion Nebula, the dark arc limiting to the north the OMC-1 South region, $\approx 45^{\prime \prime}$ to the southwest of the Trapezium. Since the dark arc also appears in the $20 \mathrm{~cm}$ continuum image, it is not due to foreground extinction but rather traces a curved part of the ionization front seen edge-on, similar to the linear "Orion bar" (O'Dell \& Yusef-Zadeh 2000). It is remarkable to find to the north of the dark arc (i.e., on the "wrong side") one of the brightest $([N]=2.74 \mathrm{mag})$ and reddest $(L-N \simeq 4.3, N-Q \simeq 2$.1) sources of our survey. This object can still be embedded within the high-density region creating the dark arc, if one assumes that the arc traces the closest part of a neutral cloud whose surface is not exactly edge-on but rather tilted with respect to our vantage point. As we have mentioned above, Smith et al. (2004) consider this bright object as the most likely driving source for the HH 202/ HH 528 outflow system.

\subsection{Point Sources}

\subsubsection{Mid-Infrared Variability}

For several $10 \mu \mathrm{m}$ sources, the comparison of the photometric results obtained in 1998 November versus 2000 December suggests the presence of mid-IR variability. In Figure 21 we plot against each other the data for the two epochs, listed in Table 3. Whereas the average magnitude difference between the two runs is less than $0.02 \mathrm{mag}$, the average scatter is $\simeq 0.27 \mathrm{mag}$, much larger than the average measure error $\Delta[N] \simeq 0.11 \mathrm{mag}$.

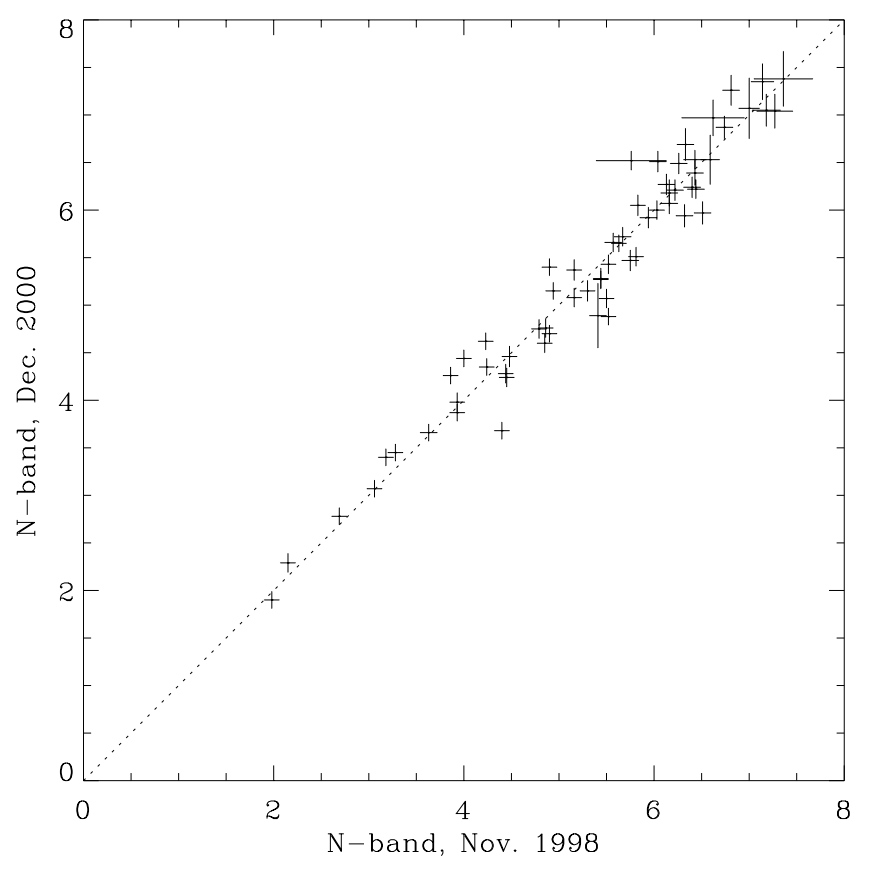

FIG. 21.-Comparison of the MAX $[N]$-band photometry at the two epochs.

This discrepancy is too large to be explained by measurement errors unaccounted for. In fact, for this type of comparison our photometric errors are probably overestimated, since they include, added in quadrature, the uncertainties on the absolute zero point. These are systematic shifts common to all magnitudes taken on the same night, which we conservatively estimated to be on the order of $0.05-0.07 \mathrm{mag}$ at $10 \mu \mathrm{m}$. Since almost all the data presented in Figure 21 were taken in two nights, 1998 November 27 and 2000 January 18, and since the systematic differences appear to be less than $0.02 \mathrm{mag}$, our zero-point uncertainties may actually be too generous, hardening the evidence for variability.

Stellar variability should be common among these sources (Herbst et al. 2002). Future comparative studies of the optical and mid-IR variability, based on simultaneous multiwavelength photometric monitoring, may allow us to understand whether the variability is due to disk activity, e.g., fluctuations of the mass accretion rates, or to changes in the foreground extinction due to inhomogeneities of the circumstellar environment or of the circumstellar disk itself.

\subsubsection{Mid-Infrared Excess}

To investigate the nature of the mid-IR emission of our sources, we compare in Figure 22 the $[K-L]$ versus $[N-Q]$ color indexes of our Trapezium stars against a sample of classical T Tauri stars in the Taurus-Auriga association. For the Trapezium stars, we complement our photometry with the $K$ - and $L$-band photometry of Muench et al. (2002), using two different symbols to indicate the $10 \mu \mathrm{m}$ data obtained in the 1998 and 2000 observing runs.

The Taurus-Auriga sources are those listed in Table 2 of Strom et al. (1989). Their $K$ - and $L$-band photometry is the average of the values available for each filter in the recent literature, mined using the VizieR Service, ${ }^{7}$ and in general turns out very close to the photometry of Strom et al. (1989).

\footnotetext{
${ }^{7}$ VizieR Service is a joint effort of CDS and ESA-ESRIN, available on the Internet at http://vizier.u-strasbg.fr/viz-bin/VizieR.
} 


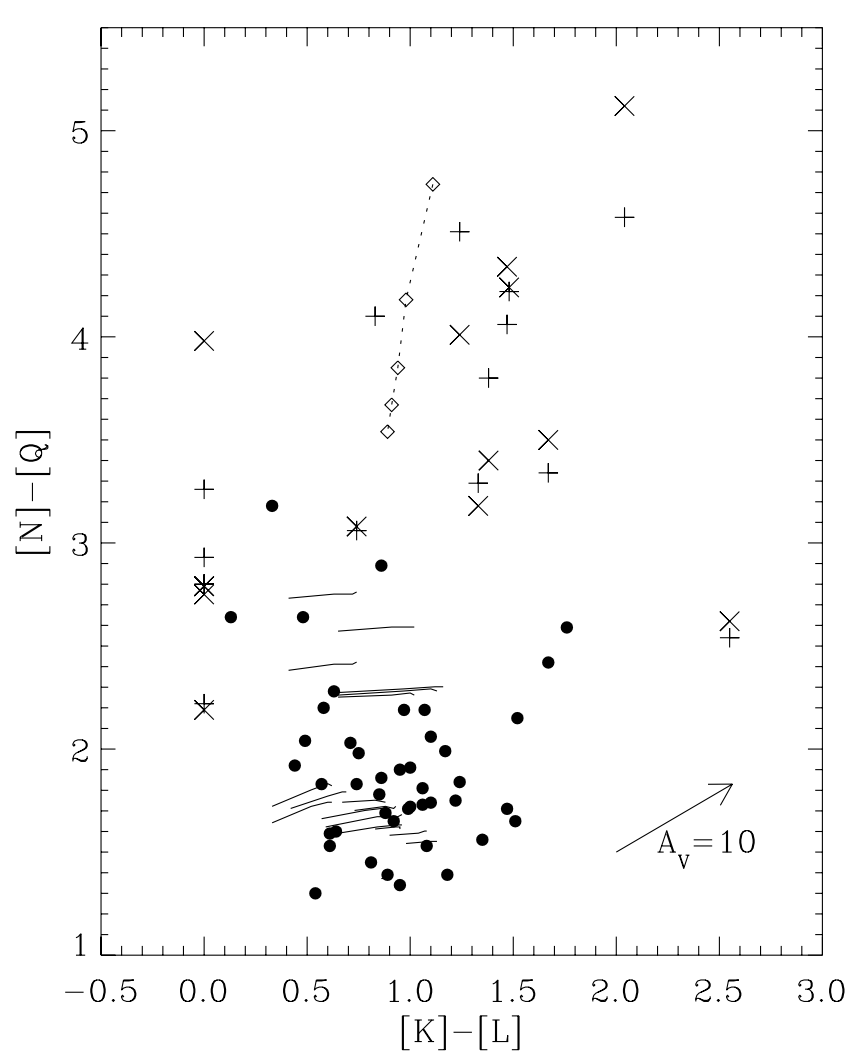

FIG. 22.-Mid-IR color-color plot for the Orion and Taurus-Auriga sources. The $K$ - and $L$-band data for Orion are from Muench et al. (2002). The crosses and plus signs refer to the MAX $10 \mu \mathrm{m}$ data taken in 1998 and 2000, respectively. Filled circles are for the Taurus data set. Solid lines represent the color of the Calvet et al. (1992) flaring disk models. The diamonds and the relative dotted line represent the model of Robberto et al. (2004) for a photoevaporated disk in an $\mathrm{H}$ II region oriented face-on with respect to both the ionizing star and the observer. Each diamond refers to a different distance from the ionizing star; from top to bottom: $30^{\prime \prime}, 60^{\prime \prime}, 90^{\prime \prime}, 120^{\prime \prime}$, and $150^{\prime \prime}$, corresponding to $0.065,0.13,0.20,0.26$, and $0.33 \mathrm{pc}$, respectively, at the assumed distance $d=450 \mathrm{pc}$ of the Orion Nebula.

The 10 and $20 \mu \mathrm{m}$ photometry is derived from the IRAS photometry at 12 and $25 \mu \mathrm{m}$ in the following way. The IRAS PSC2 fluxes are quoted at the effective wavelengths assuming an input energy distribution constant per $\log \nu$, i.e., $\nu F_{\nu}=$ constant. This is also the shape of the typical "flat" spectral energy distribution (SED) of circumstellar disks around T Tauri stars. We therefore assume the IRAS fluxes to be colorcorrected and adjust their values only to account for the difference in effective wavelength between the filters. From the $\nu F_{\nu}=$ constant condition, we derive $m(10)=m(12)+0.516$ and $m(20)=m(25)+0.694$ using the zero-magnitude fluxes reported in $\S 2$ of this paper and in the IRAS PSC 2 manual. We have visually inspected each SED plotting, together with all the IR data available in the literature and our interpolated 10 and $20 \mu \mathrm{m}$ points. Whereas our points are always in excellent agreement with the overall shape of the SED (admittedly constrained by the IRAS points), most of the 10 and $20 \mu \mathrm{m}$ groundbased data are old and suspiciously far from the best-fit line.

Figure 22 clearly shows that there is a systematic difference between the Orion and the Taurus-Auriga samples, as the Orion stars have an $[N-Q]$ excess between 3 and $5 \mathrm{mag}$, whereas for Taurus-Auriga the $[N-Q]$ index ranges between 1 and 3 mag. In addition, the $[K-L]$ excesses appear on average higher for Orion, although for both samples the majority of stars have $[K-L]$ in the range $0.7-1.5 \mathrm{mag}$. We also show in
Figure 22 five stars without $L$-band photometry but well detected at shorter wavelengths, to which we have assigned $[K-L]=0$. For these sources as well the $[N-Q]$ index appears higher than that of the stars in Taurus-Auriga.

Extinction cannot explain these effects, either in terms of intensity or reddening. In Figure 22 we have indicated with an arrow the reddening corresponding to $A_{v}=10 \mathrm{mag}$ of extinction. Hillenbrand (1997) suggests that the mean extinction for the optically visible population of the Orion Nebula cluster is $A_{V} \lesssim 2.5 \mathrm{mag}$, with few optical stars having $A_{V} \gtrsim 5 \mathrm{mag}$. For the IR sample, the mean extinction is approximately 3 times higher, but only a small fraction of sources have $A_{V} \gtrsim 15 \mathrm{mag}$ (Hillenbrand \& Hartmann 1998).

Calvet et al. (1992) have calculated the IR colors of accreting disks around classical T Tauri stars assuming a variety of stellar and disk parameters. Each one of their models is represented in Figure 22 as a solid line, as different orientation angles are taken into account. The Calvet et al. (1992) predictions fall in general within the region of the observed $\mathrm{T}$ Tauri star colors. In particular, the models with the highest $[N-Q] \gtrsim 2$ have in common a higher value of $\gamma$, the index of the power law describing the increase of the disk height with the distance from the central star. Large disk flaring angles could therefore explain the extreme $[N-Q]$ colors of our sources.

In a previous paper (Robberto et al. 2002), we have explored the continuum SED of circumstellar disks embedded within an $\mathrm{H}$ II region like Orion. We predicted that the extra energy input from the ionizing stars and from the nebular environment raises the disk temperature and causes an increase of the disk flaring angle. An inflated disk will also intercept a larger fraction of the flux emitted by its own central star. In the vicinity of $\theta^{1}$ Ori $\mathrm{C}$, the neutral flow produced by disk photoevaporation originates a neutral envelope bordered by a ionization front, the "proplyd" phenomenon (O'Dell et al. 1993; O'Dell \& Wen 1994). Together with the disk, the dusty envelope also contributes to the mid-IR emission. Our models show that there is in general a strong enhancement of the mid-IR emission due both to the higher disk temperature and the presence of the photoevaporated envelope. The UV-heated dust produces a characteristic far-IR peak of emission at 30-60 $\mu \mathrm{m}$. In Figure 22 we show as a dotted line the locus of the colors resulting from our model, assuming the limiting case of a disk facing both the ionizing star (for simplicity $\theta^{1}$ Ori $C$ is considered the only ionizing source) and the Earth at various distances from $\theta^{1}$ Ori C. The $[N-Q]$ colors predicted by this model nicely match those of the Orion sample, supporting the hypothesis that the UV radiation is ultimately responsible for the strong enhancement of $20 \mu \mathrm{m}$ emission.

In Robberto et al. (2002), we have shown that the IR SEDs of proplyds depend on a number of factors, both physical, such as the distance from the ionizing star, the size of the envelope, the inner disk radius, the temperature of the central star, and the dust composition, and geometrical, such as the tilt angle of the disk with respect to the ionizing star and to the Earth. The projection factors in particular have a dramatic impact on the observed SED, since disk surfaces at the same distance from $\theta^{1}$ Ori $\mathrm{C}$ can be exposed to very different UV fluxes and appear from the Earth under all possible angles, showing either the front or the back side. The 30-60 $\mu \mathrm{m}$ peak stands as the main observational characteristic of these systems. Depending on the dust temperature and grain size, IR color indexes that do not include the $20 \mu \mathrm{m}$ band, such as $[K-N]$ or $[L-N]$, may remain unaffected by a mid-IR peak too cold to produce strong excess at $10 \mu \mathrm{m}$. For the main group of sources detected only at 
$10 \mu \mathrm{m}$, it is necessary to analyze in detail each individual source to constrain the disk properties. This analysis will be presented in a second paper and will include the result of our deep $10 \mu \mathrm{m}$ survey of selected fields in Orion.

\section{CONCLUSION}

Using a new restoration method developed for this project, we have obtained the first wide-field and diffraction-limited 10 and $20 \mu \mathrm{m}$ images of the core of the Orion Nebula. The images confirm that the morphology of the Orion Nebula at mid-IR wavelengths is dominated by the $\mathrm{BN} / \mathrm{KL}$ complex and by the Ney-Allen Nebula. At lower signal levels, the Orion Nebula shows a filamentary structure that traces the walls of the $\mathrm{H}$ II region. A remarkable group of arclike structures is detected $\approx 1^{\prime}$ to the south of the Trapezium. We analyze the structure of the Ney-Allen Nebula in the framework of analytic wind-wind interaction models, finding evidence for a complex kinematical status at the center of the cluster. The B $0.5 \mathrm{~V}$ star $\theta^{1}$ Ori D, associated with the brightest mid-IR structure of the Trapezium region, is probably surrounded by a photoevaporated circumstellar disk. The fact that, among the main four Trapezium stars, this star is the only one without a binary companion suggests that stellar multiplicity and massive circumstellar disks are mutually exclusive in the Trapezium. We detect in the OMC-1 South region three point sources that can be associated with the mass-loss activity detected at millimeter wavelengths. Finally, we list the position and photometry of 177 point sources, the large majority of which have been detected for the first time in the mid-IR. Twenty-two of them lack counterparts at shorter wavelengths and are therefore candidates for deeply embedded protostars. The comparison of photometric data obtained at two different epochs reveals that source variability at $10 \mu \mathrm{m}$ is present up to a level of $\approx 1 \mathrm{mag}$ on a timescale of $\sim 2$ yr. We compare the mid-IR color-color diagram for our sources in Orion and a similar data set in the Taurus association, finding indications for large mid-IR excess, especially at $20 \mu \mathrm{m}$, and discuss this finding in the framework of the Robberto et al. (2002) model.

We have also presented narrowband images of the $\mathrm{BN} / \mathrm{KL}$ complex taken in the standard silicate filter set. The BN/KL complex is resolved into multiple and extended features. In particular, we resolve a point source in correspondence of the IRc2-A knot. We derive the extinction and temperature maps on the basis of the simple "two-component" model for the IR emission and discuss the shape of the silicate absorption feature and possible scenarios for dust evolution in the $\mathrm{H}$ II region.

The authors are indebted to the MPIA and UKIRT staff for their support with the MAX operations. The contribution of $\mathrm{M}$. Cohen to the MAX calibration is acknowledged. We thank Bob O'Dell for several useful comments and in particular for insights on the interpretation of the OMC-1 South region. Comments from an anonymous referee also greatly contributed to improve the original manuscript. S. G. P.'s visit at STScI was supported by the STScI 2002 Summer Student Program.

\section{REFERENCES}

Aitken, D. K., Bailey, J. A., Roche, P. F., \& Hough, J. M. 1985, MNRAS, 215, 815

Aitken, D. K., Roche, P. F., Spenser, P. M., \& Jones, B. 1981, MNRAS, 195, 921

Aitken, D. K., Smith, C. H., Moore, T. J. T., Roche, P. F., Fujiyoshi, T., \& Wright, C. M. 1997, MNRAS, 286, 85

Bally, J., O’Dell, C. R., \& McCaughrean, M. J. 2000, AJ, 119, 2919

Bally, J., Sutherland, R. S., Devine, D., \& Johnstone, D. 1998, AJ, 116, 293

Becklin, E. E., \& Neugebauer, G. 1967, ApJ, 147, 799

Bertero, M., \& Boccacci, P. 1998, Introduction to Inverse Problems in Imaging (Bristol: IoP Publishing)

Bertero, M., Boccacci, P., Di Benedetto, F., \& Robberto, M. 1999, Inverse Problems, 15, 345

Bertero, M., Boccacci, P., \& Robberto, M. 1998, Proc. SPIE, 3354, 877 2000, PASP, 112, 1121

Bessell, M. S., \& Brett, J. M. 1988, PASP, 100, 1134

Bowey, J. E. 1998, Ph.D. thesis, Univ. Central Lancashire

Bowey, J. E., Adamson, A. J., \& Yates, J. A. 2003, MNRAS, 340, 1173

Calvet, N., Magris, G. C., Patino, A., \& D'Alessio, P. 1992, Rev. Mex. AA, 24, 27

Cantó, J., Raga, A. C., \& Wilkin, F. P. 1996, ApJ, 469, 729

Castor, J., McCray, R., \& Weaver, R. 1975, ApJ, 200, L107

Chelli, A., Perrier, C., \& Lena, P. 1984, ApJ, 280, 163

Chrisostomou, A., Gledhill, T. M., Ménard, F., Hough, J. H., Tamura, M., \& Bailey, J. 2000, MNRAS, 312, 103

Churchwell, E., Felli, M., Wood, D. O. S., \& Massi, M. 1987, ApJ, 321, 516

Day, K. L., \& Donnn, B. D. 1978, ApJ, 222, L45

Doi, T., O’Dell, C. R., \& Haritgan, P. 2004, AJ, 127, 3456

Dougados, C., Léna, P., Ridgway, S. T., Christou, J. C., \& Probst, R. G. 1993, ApJ, 406, 112

Downes, D., Genzel, R., Becklin, E. E., \& Wynn-Williams, C. G. 1981, ApJ, 244,869

Draine, B. T. 1985 , ApJS, 57, 587

Draine, B. T., \& Lee, H. M. 1984, ApJ, 285, 89

Forrest, W. J., Gillett, F. C., \& Stein, W. A. 1975, ApJ, 195, 423

García-Arredondo, F., Henney, W. J., \& Arthur, S. J. 2001, ApJ, 561, 830

Gaume, R. A., Wilson, T. L., Vrba, F. J., Johnston, K. J., \& Schmid-Burgk, J. 1998, ApJ, 493, 940

Genzel, R., Reid, M. J., Moran, J. M., \& Downes, D. 1981, ApJ, 244, 884

Genzel, R., \& Stutzki, J. 1989, ARA\&A, 27, 41
Gezari, D. Y., Backman, D. E., \& Wener, M. D. 1998, ApJ, 509, 283

Greenhill, L. J., Gezari, D. Y., Danchi, W. C., Najita, J., Monnier, J. D., \& Tuthill, P. G. 2004, ApJ, 605, L57

Hanner, M. S., Brooke, T. Y., \& Tokunaga, A. T. 1995, ApJ, 438, 250

Hawarden, T., et al. 1999, Proc. SPIE, 3785, 82

Hayward, T. L. 1994, ApJ, 429, 726

Hayward, T. L., Houck, J. R., \& Miles, J. W. 1994, ApJ, 433, 157

Henney, W., \& Arthur, S. J. 1998, AJ, 116, 322

Henney, W., \& O’Dell, C. R. 1999, AJ, 118, 2350

Henney, W. J., O'Dell, C. R., Meaburn, J., Garrington, S. T., \& Lopez, J. A. 2002, ApJ, 566, 315

Herbst, W., Bailer-Jones, C. A. L., Mundt, R., Meisenheimer, K., \& Wackermann, R. 2002, A\&A, 396, 513

Hillenbrand, L. A. 1997, AJ, 113, 1733

Hillenbrand, L. A., \& Carpenter, J. M. 2000, ApJ, 540, 236

Hillenbrand, L. A., \& Hartmann, L. 1998, ApJ, 492, 540

Jäger, C., Mutschke, H., Begemann, B., Dorschner, J., \& Henning, T. 1994, A\&A, 292, 641

Johnston, K. J., Migenes, V., \& Norris, R. P. 1989, ApJ, 341, 847

Johnstone, D., Hollenbach, D., \& Bally, J. 1998, ApJ, 499, 758

Jones, B. F., \& Walker, M. F. 1985, AJ, 90, 1320

Keene, J., Hildebrand, R. H., \& Whitcomb, S. E. 1982, ApJ, 252, L11

Kleinmann, D. E., \& Low, F. J. 1967, ApJ, 149, L1

Lada, C. J., \& Lada, E. A. 2003, ARA\&A, 41, 57

Lada, C. J., Muench, A. A., Haisch, K. E., Jr., Lada, E. A., Alves, J. F., Tollestrup, E. V., \& Willner, S. P. 2000, AJ, 120, 3162

Laques, P., \& Vidal, J.-L. 1979, A\&A, 73, 97

Lonsdale, C. J., Becklin, E. E., Lee, T. J., \& Stewart, J. M. 1982, AJ, 87, 1819

Lucas, P. W., \& Roche, P. F. 2000, MNRAS, 314, 858

Luhman, K. L., Rieke, G. H., Young, E. T., Cotera, A. S., Chen, H., Rieke, M. J., Schneider, G., \& Thompson, R. I. 2000, ApJ, 540, 1016

Mathis, J. S. 1990, ARA\&A, 28, 37

McCaughrean, M., \& Gezari, D. 1991, in ASP Conf. Ser. 14, Astrophysics with Infrared Arrays, ed. R. Elston (San Francisco: ASP), 301

McCaughrean, M. J., Rayner, J., Zinnecker, H., \& Stauffer, J. 1996, in Disks and Outflows around Young Stars, ed. S. Beckwith, J. Staude, A. Quetz, \& A. Natta (Berlin: Springer), 33

McCaughrean, M. J., \& Stauffer, J. R. 1994, AJ, 108, 1382

McCullough, P. R., Fugate, R. Q., Christou, J. C., Ellerbroek, B. L., Higgins, C. H., Spinhirne, J. M., Cleis, R. A., \& Moroney, J. F. 1995, ApJ, 438, 394 Meaburn, J. 1988, MNRAS, 233, 791 
Menten, K. M., \& Reid, M. J. 1995, ApJ, 445, L157

Mezger, P. G., Wink, J. E., \& Zylka, R. 1990, A\&A, 228, 95

Muench, A. A., Lada, E. A., Lada, C. J., \& Alves, J. 2002, ApJ, 573, 366

Ney, E. P., \& Allen, D. A. 1969, ApJ, 155, L193

O’Dell, C. R. 2001a, ARA\&A, 39, 99 . 2001b, PASP, 113, 29

O’Dell, C. R., \& Doi, T. 2003a, AJ, 125, 277 2003b, AJ, 125, 2753

O’Dell, C. R., \& Wen, Z. 1994, ApJ, 436, 194

O’Dell, C. R., Wen, Z., \& Hu, X. 1993, ApJ, 410, 696

O’Dell, C. R., \& Wong, K. 1996, AJ, 111, 846

O’Dell, C. R., \& Yusef-Zadeh, F. 2000, AJ, 120, 382

Panagia, N. 1975, A\&A, 42, 139

Preibisch, T., Balega, Y., Hofmann, K., Weigelt, G., \& Zinnecker, H. 1999, NewA, 4, 531

Prosser, C. F., Stauffer, J. R., Hartmann, L., Soderblom, D. R., Jones, B. F., Werner, M. S., \& McCaughrean, M. J. 1994, ApJ, 421, 517

Rieke, G. H., \& Lebofsky, M. J. 1985, ApJ, 288, 618

Rieke, G. H., Low, F. J., \& Kleinmann, D. E. 1973, ApJ, 186, L7

Rinehart, S. A., Hayward, T. L., \& Houck, J. R. 1998, ApJ, 503, 415

Robberto, M., Beckwith, S. V. W., \& Panagia, N. 2002, ApJ, 578, 897

Robberto, M., \& Herbst, T. M. 1998, Proc. SPIE, 3354, 711

Robberto, M., Song, J., Mora Carrillo, G., Beckwith, S. V. W., Makidon, R. B., \& Panagia, N. 2004, ApJ, 606, 952

Roche, F. P., \& Aitken, D. K. 1984, MNRAS, 208, 481

Rodríguez-Franco, A., Martín-Pintado, J., \& Wilson, T. L. 1999a, A\&A, 344, L57

1999b, A\&A, 351, 1103

Rosado, M., de la Fuente, E., Arias, L., Raga, A., \& Le Coarer, E. 2001, AJ, 122,1928

Schmid-Burgk, J., Güsten, R., Mauersberger, R., Schulz, A., \& Wilson, T. L. 1990, ApJ, 362, L25
Scoville, N., Kleinmann, S. G., Hall, D. N. B., \& Ridgway, S. T. 1983, ApJ, 275, 201

Shuping, R. Y., Morris, M., \& Bally, J. 2004, AJ, 128, 363

Sloan, G. C., Hayward, T. L., Bregman, J. D., \& Allamandola, L. J. 1996, BAAS, 28, 1417

Smith, N., Bally, J., Shuping, R. Y., Morris, M., \& Hayward, T. L. 2004, ApJ, 610, L117

Stahler, S. W., Palla, F., \& Ho, P. T. P. 2000, in Protostars and Planets IV, ed. V. Mannings, A. P. Boss, \& S. S. Russell (Tucson: Univ. Arizona Press), 327

Strom, K. M., Strom, S. E., Edwards, S., Cabrit, S., \& Skrutskie, M. F. 1989, AJ, 97, 1451

Tan, J. C. 2004, in ASP Conf. Ser. 323, Star Formation in the Interstellar Medium, ed. D. Johnstone, F. C. Adams, D. N. C. Lin, D. A. Neufeld, \& E. C. Ostriker (San Francisco: ASP), 249

Varosi, F., \& Gezari, D. Y. 1993, in ASP Conf. Ser. 52, Astronomical Data Analysis Software and Systems II, ed. R. J. Hanisch, R. J. V. Brissenden, \& J. Barnes (San Francisco: ASP), 393

Weaver, R., McCray, R., Castor, J., Shapiro, P., \& Moore, R. 1977, ApJ, 218, 377

Werner, M. W., Dinerstein, H. L., \& Capps, R. W. 1983, ApJ, 265, L13

Whittet, D. C. B., Bode, M. F., Longmore, A. J., Adamson, A. J., McFadzean, A. D., Aitken, D. K., \& Roche, P. F. 1988, MNRAS, 233, 321

Wilson, T. L., Gaume, R. A., Gensheimer, P., \& Johnston, K. J. 2000, ApJ, 538, 665

Wynn-Williams, C. G., Genzel, R., Becklin, E. E., \& Downes, D. 1984, ApJ, 281,172

Zapata, L. A., Rodríguez, L. F., Kurtz, S. E., O’Dell, C. R., \& Ho, P. T. P. 2004, ApJ, 610, L121

Ziurys, L. M., Martin, R. N., Pauls, T. A., \& Wilson, T. L. 1981, A\&A, 104, 288

Ziurys, L. M., Wilson, T. L., \& Mauersberger, R. 1990, ApJ, 356, L25 